\title{
RELATIONAL EFFECTS OF DATA NETWORKS: HOW STRATEGICALLY ALIGNED DATA NETWORKS IMPACT DIGITAL TRANSFORMATION
}

\author{
A Dissertation \\ Submitted to \\ The Temple University Graduate Board \\ In Partial Fulfillment \\ of the Requirements for the Degree \\ DOCTOR OF BUSINESS ADMINISTRATION \\ by \\ Michael Pierre Toussaint \\ December 2020 \\ Examining Committee Members: \\ Detmar W. Straub, Ph.D., Advisory Chair, Management Information Systems \\ Susan Mudambi, Ph.D., Marketing and Supply Chain Management \\ Anthony Vance, Ph.D., Management Information Systems \\ Charles Dhanaraj, Ph.D., External Member, University of Denver
}


Copyright

2020

by

Michael Pierre Toussaint

All Rights Reserved 


\section{EXTENDED ABSTRACT}

Digital Transformation (DX) has become a pervasive global phenomenon that is having a profound effect at all levels of an enterprise. The umbrella term DX is supported by nascent technologies such as blockchain, Internet of Things (IoT), and cloud-based computing and networking. The speed by which these new DX technologies have emerged has challenged current technical infrastructures, budgets, and skillsets as organizations attempt to incorporate and implement these technologies as part of pervasive digital operational initiatives. DX also includes the transformative effects (deployment and adoption) of these technologies, and these are referred to as "outcomes" in this dissertation.

Not surprisingly, recent studies indicate that at least seventy percent of DX projects either fail or underperform. As firms assess existing technology-to-business alignments in search of attributable causation, they discover that these alignments are often opaque regarding the capabilities required to obtain optimal digital transformation outcomes from the application of specific technologies. This is especially true as underlying technology infrastructures and architectures, which have had a traditionally functional role such as the data network, are increasingly relied upon to support the strategic outcome requirements of DX.

This dissertation uses an inductive, multiple case study approach to explore these relationships and outcomes. It directly observes a set of large organizations across multiple verticals. These organizations have all completed pervasive digital transformation initiatives, more specifically, this study measured the resultant levels of 
digital transformation project outcomes achieved as a fraction of the initial digital transformation project deliverables. Furthermore, the study makes inferences regarding the relationship of the data network deployment posture to the observed digital project outcomes.

To study this empirically, data network alignment is classified as either being functionally or strategically aligned. Analysis of the resultant data revealed four distinct themes: (a) organizations that procured data network equipment with pricing as the critical determinant experienced sub-par digital transformation outcomes; (b) organizations which considered key business drivers when procuring and architecting the data network achieved more successful digital transformation outcomes; (c) organizations which did not perform a network upgrade or perform a significant data network architectural change during the previous five years did not meet their own goals of digital transformation, and (d) some DX technology deployments achieved a high percentage of project deliverables without undertaking a data network upgrade.

With respect to the fourth theme, however, the resulting low internal adoption of deployed DX technology among targeted operational units resulted in a 'split-brain' operation and thus an overall underperformance of the DX project.

The final dissertation chapter includes recommendations for future qualitative research on both digital transformation outcomes and the effect of managed network services on digital transformation outcomes as well as the need for quantitative research to establish deeper causation beyond the loose causation which is posited in this paper. 


\section{ACKNOWLEDGEMENTS}

It would have been impossible to attempt, much less complete, this dissertation without the support of my loving wife Aimee, my son Tariq, and my twin "Little Piggies" Gianna and Adriana. They sacrificed countless days, nights, and weekends with me so that I could concentrate fully on completing my doctoral journey. I am forever grateful for all they have done so that I could realize this dream. I owe them a debt that I can never repay, but I begin by dedicating this dissertation to them.

I am grateful to the organizations represented in this paper which allowed me to spend time on-site with their respective teams. Embedding oneself with the object of one's study and observing the phenomena directly is at once extremely challenging and extremely rewarding.

I would also like to thank my mentor Dr. Detmar Straub, whose wonderful talent for making even the most complex subject matter approachable and under whose continued guidance this paper came to fruition. I am also thankful to my dissertation committee; whose constant encouragement was vital to completing this paper.

It has been a challenging, yet rewarding, journey as I juggled the requirements of family, friends, and the demands of writing both a dissertation and a steady cadence of research notes as a senior Gartner Analyst. Alas, it is a marathon not a sprint, and through all the ups and downs along the way there is no greater feeling than seeing this dissertation transformed from only the fleeting firings of a few neurons to a tangible object which has, hopefully, grown to be greater than the sum of its parts. 
TABLE OF CONTENTS

Page

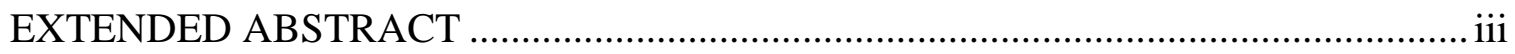

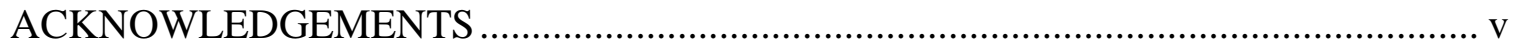

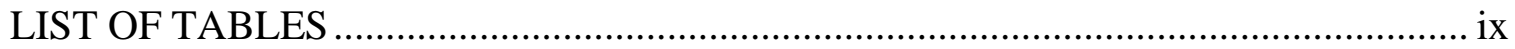

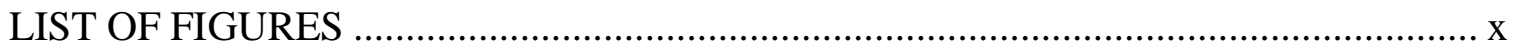

\section{CHAPTER}

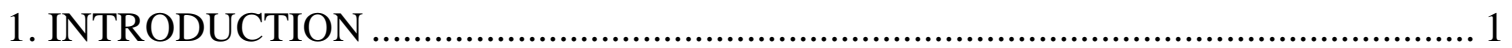

Explaining "Digital” with "Analog” Models ............................................................. 3

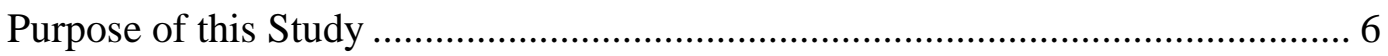

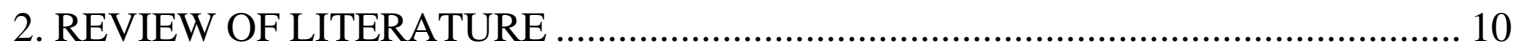

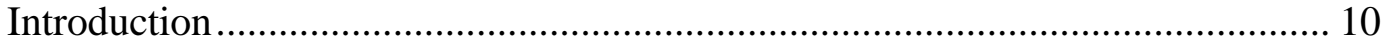

Information Technology Investments and Performance Outcomes...................... 12

Deriving Value from IT Investments w/r/t Functional Technologies .................... 13

Identifying and Classifying Functional and Strategic Technologies .................... 16

Organization and Information Technology Strategic Alignment ......................... 18

The Fourth Industrial Revolution, Digital Transformation.................................... 21

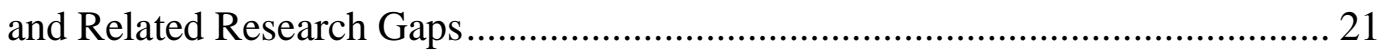

Technology Models and Frameworks in a Transforming Landscape................... 28

Information Technology Adoption ...................................................................... 33

Conceptual Model: Impact of Alignment of the Data Network on DX Outcomes

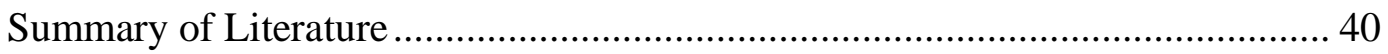

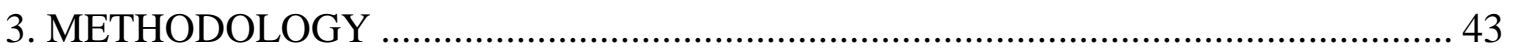

Rationale for Research Approach .................................................................... 44

How the Selected Method Addresses the Research Questions .............................. 47

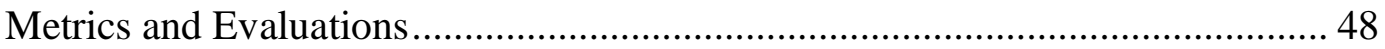

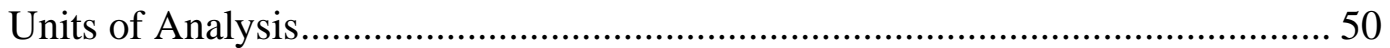

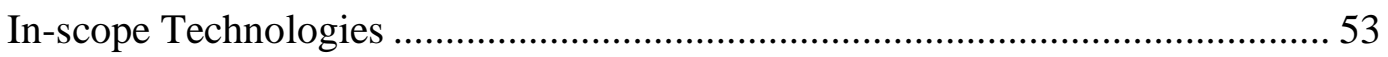

Pilot and Multiple Case Studies Participant Sources ........................................... 53

Confidentiality of Data .................................................................................. 55 
Techniques for Data Collection and Participant Observation............................. 56

Instrumentation of Interview Schedules/Scripts ............................................ 59

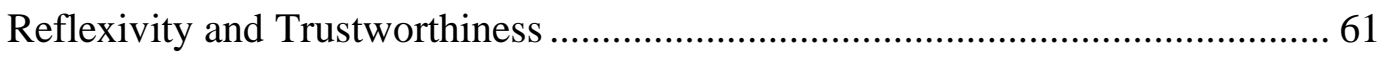

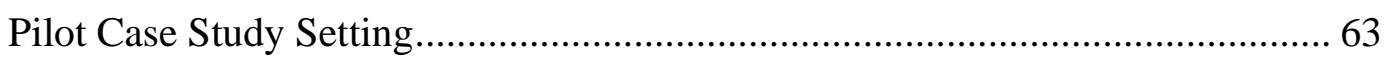

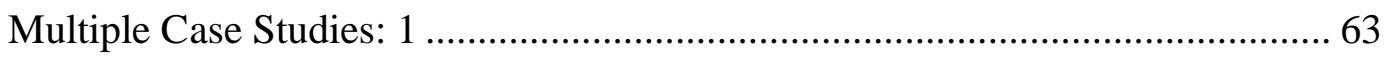

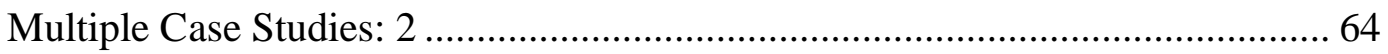

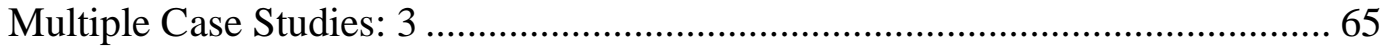

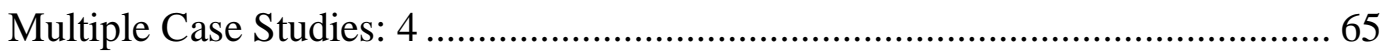

4. PILOT CASE STUDY - AN INSTITUTION OF HIGHER EDUCATION LEARNS A

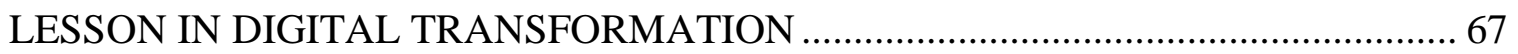

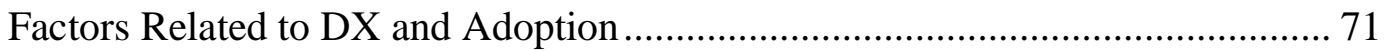

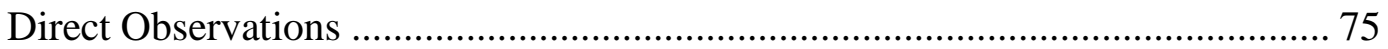

5. CASE STUDY 1 - A RETAIL FIRM IN THE U.S. HEARTLAND MAKES

DIGITAL TRANSFORMATION THE HEART OF ITS OPERATIONS .................... 79

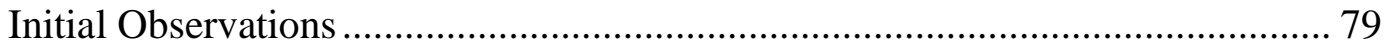

Digital Transformation Technology Team Preparedness ................................. 84

DX Project Kickoff and Stakeholder Inclusion .......................................... 85

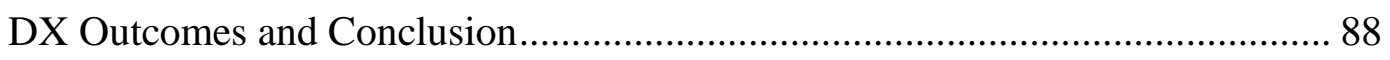

6. CASE STUDY 2 - AN AUTOMOTIVE FIRM ATTEMPTS TO DRIVE PROFITS

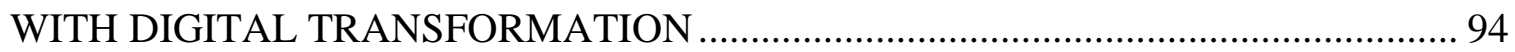

The Factors for Digitalization in the Automobile Market ................................ 94

The Best Laid Plans of Mice and Men Often Go Awry ................................... 98

Patterns Emerge from Interviews............................................................ 98

Digital Transformation Outcome and Conclusion .......................................... 101

7. CASE STUDY 3 - TOO BIG TO FAIL AT DIGITAL TRANSFORMATION ....... 103

Digital Transformation Outcome and Conclusion .......................................... 108

8. CASE STUDY 4 - AN AIRLINE STRUGGLEs TO GAIN MARKET ALTITUDE

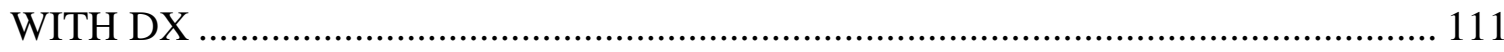

Digital Transformation Outcome and Conclusion ......................................... 119

9. COMBINATORIAL OBSERVATIONS AND RESULTS ................................... 120

10. DISCUSSION, IMPLICATIONS, RECOMMENDATIONS FOR FURTHER STUDY, AND CONCLUSION ...................................................................... 128

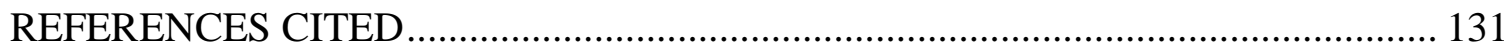


APPENDICES

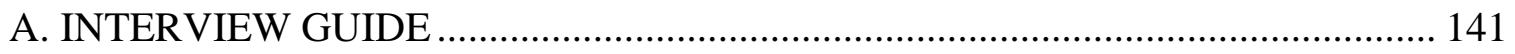

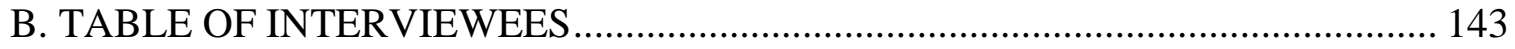

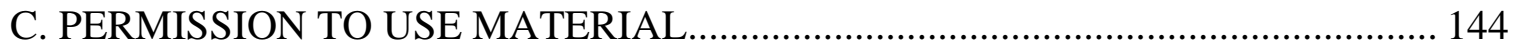




\section{LIST OF TABLES}

Table

Page

1. Sampling of Previous Literature on Digital Transformation .25

2. Selected Literature on Models of Strategic Technology Alignment / Adoption-to-

Business Requirements.

3. Most Preeminent Information Technology Adoption Models (Hameed et al., 2012) ...34

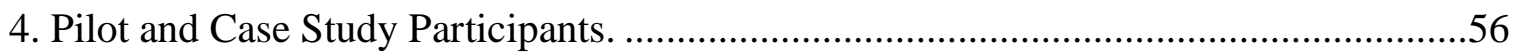

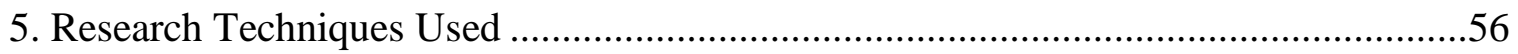

6. Case Study Tactics for Four Design Tests (Yin, 2013) ...........................................58

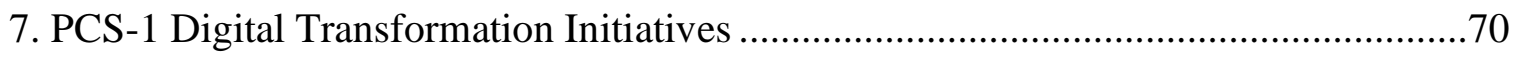

8. PCS-1 Data Network Preparedness to support DX Sample Responses........................75

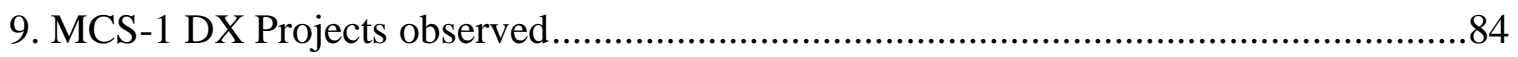

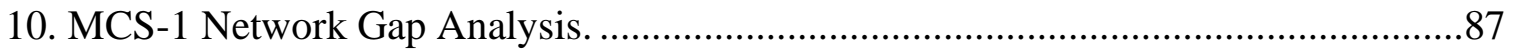

11. MCS-2 DX Projects and Network Dependencies ..............................................99

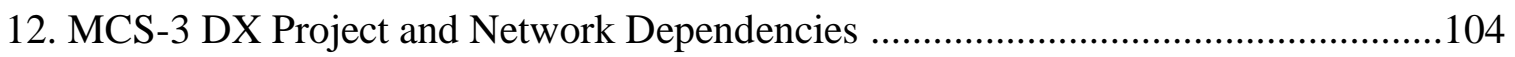

13. MCS-4 Data Network Reliant DX Projects .......................................................116

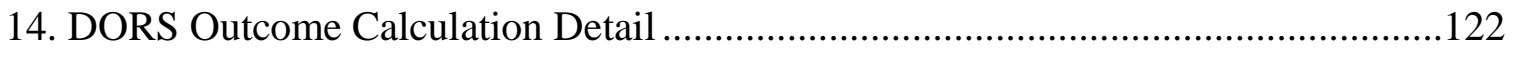

15. Comparative Multiple Case Study Results ...........................................................124

16. Keywords indicating Strategic or Tactical Data Network Alignment. ....................125

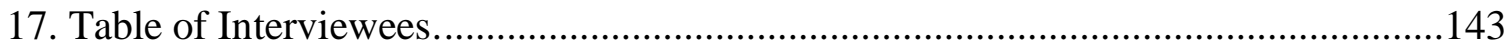




\section{LIST OF FIGURES}

Figure $\quad$ Page

1. Conceptual Model of DX, Data Network Alignment, and DX Outcomes ................... 39

2. Positivist Qualitative Methods............................................46

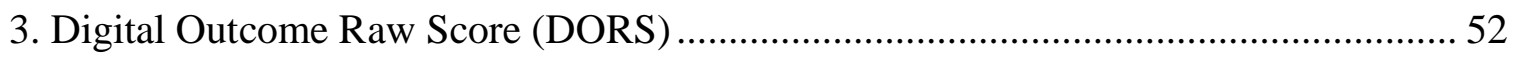

4. PCS-1 Relational view of Network-to-DX Projects ............................................ 77

5. MCS-1 Relational view of Network-to-DX Projects ............................................ 90

6. MCS-2 Relational view of Network-to-DX Projects........................................... 101

7. MCS-3 Relational view of Network-to-DX Projects............................................ 105

8. MCS-4 Relational view of Network-to-DX Projects .......................................... 118 


\section{CHAPTER 1}

\section{INTRODUCTION}

A revolution is upon us, and as Gill Scott-Heron states in his epic poem "The Revolution Will Not Be Televised," the revolution is of epic relevance. It will be pervasive, in this case affecting all organizations of all sizes and verticals, on a global scale.

In 2015, Klaus Schwab studied and named the sweeping disruption: the fourth industrial revolution. Schwab (2015) identified the hallmark of the revolution to be the pervasive connectivity requirements of new technologies such as the cloud, artificial intelligence, machine learning, and self-driving cars, to name a few (Schwab, 2015).

In 2017, Demirkan et al. identified digital transformation (DX) as a key property of the fourth industrial revolution. Research has revealed that DX is now a strategic priority for over $85 \%$ of executives in the United States and the United Kingdom (Hess et al., 2016). Indeed, DX is not just a business phenomenon; rather, it has become pervasive at all levels of society and business as new smart cyber systems are introduced and adopted on a global scale (Guoping et al., 2017).

To this end, Chalons and Dufft (2016) stated that the new digital revolution is as relevant to us as the first industrial revolution of the $19^{\text {th }}$ century was to the people of that time (Chalons and Dufft, 2016). Dermirkan et al. (2016) defined digital transformation as "the profound and accelerating transformation of business activities, processes, competencies, and models to fully leverage the changes and opportunities brought by newly available technologies and their impact across society in a strategic and prioritized way" (p. 14). To this end, a 2017 Gartner Survey indicated that $45 \%$ of CEOs have already undertaken digital transformation at some level. A 2017 survey by Bourne and Dell 
Technologies polled business leaders from 16 countries and across 12 industry verticals to understand their level of digital transformation maturity. The survey revealed that $53 \%$ of respondents have evaluated (34\%), in the process of adopting (14\%), or have fully adopted (5\%) digital transformation within their respective organizations (Bourne et al., 2017).

For this dissertation, a working definition of DX is requisite. Supported by contemporary digital technologies such as artificial intelligence, blockchain, edge computing, Internet of Things (IoT), and the cloud, digital transformation is the strategic restructuring of organizational processes to be able to deliver products and services more seamlessly, transparently, efficiently, and effectively to stakeholders. Importantly, DX also includes the transformative effects (deployment and adoption) of these technologies, and these are referred to as "outcomes" in this dissertation.

As with any revolution, the way forward is often unclear. Pervasive change is not a straight, well-lit superhighway; rather, it is a dark and winding narrow road, and in many cases, there simply is no road at all! In fact, Buytendijk (2017), an analyst at research and advisory firm, Gartner, Inc., found that business leaders are often confused by the highly integrated, and cross-functionally reliant, strategy required to successfully plan for, and deploy digital transformation initiatives within their organizations. This confusion is also captured in practitioner research such as the 2016 KPMG Global Transformation Study where there is evidence to suggest that as many as $84 \%$ of digital transformation projects fail outright or fail to achieve their digital transformation goal at a medium or high level. And as nascent technologies such as blockchain, cloud networking, edge computing, and the Internet of Things (IoT) begin to define key DX 
technologies, certain skillsets, business alignment, and technological architectures must assume new shapes to successfully support DX.

Supporting this challenge, Manuputty and Wijaya (2013) stated that "without a clear plan, investment in IS/IT will run without direction" (p. 176). This point is especially pertinent as the requirement to realize new strategic organizational goals, using digital new digital technologies, begins to render previously well-understood technology adoption constructs insufficient for describing the right resources and alignments necessary to successfully adopt a deeply rooted digital organizational posture (Liu et al., 2011).

\section{Explaining "Digital” with "Analog” Models}

Academic and practitioner research on digital transformation has examined the driving factors, conditions, organizational fit, and consumption of IT, all with a functional lens focused on IT and with a strategic lens focused on business requirements (Bharadwaj et al., 2013). This is a historically analog vision (rather than a digital vision) of this transformative process. Digital transformation and adoption requires an integrative approach where technology procurement and business initiatives blend into a focused strategic direction. Historical analog visions focus their lens on technology as a mere infrastructure (a la Nicholas Carr's famous 2003 article in the Harvard Business Review entitled "IT Doesn't Matter.") In a like fashion, if the term 'digital adoption' is bound to the concept of "technology adoption,", then research in this area will be confined to no fewer than fifteen, well recognized but 'analog technology' historical models. These are of three types: (1) preeminent, comprehensive technology adoption models (see Table 2 
in the following section); (2) at least two preeminent enterprise architecture (EA) frameworks; and (3) many less-widely known models, standards, and frameworks.

Of these traditional models, the Technology Adoption Model (TAM), Diffusion of Innovation Theory (DOI), and Technology Organization and Environment (TOE) tend to be ubiquitous, with DOI being most widely used of the aforementioned to research IT adoption phenomena (Pervan et al., 2015; Hameed at al., 2012). However, all preeminent technology adoption models narrow their view primarily to individual level technology adoption while organizational level adoption research remains relatively sparse (Yu et al., 2009). Additionally, all preeminent technology adoption models predate the introduction of digital business transformation, an observation that presents an opportunity for the introduction of new models (Berman, 2012).

While business-to-IT alignment is one of the most frequently studied concepts in both industry and academic sectors, organizations utilizing traditional business strategies that have not stressed alignment have often failed to take full advantage of IT as a strategic tool (Ullah and Lai, 2013). As a result, the failure to align IT to business requirements likely results in between $\$ 50$ and $\$ 150$ billion dollars in failed IT projects each year in the United States alone (Flyvbjerg and Budzier, 2011). Despite extensive literature and research dedicated to understanding technology adoption and alignment within enterprises, the fast pace of technological change in a digital adoption regime and subsequent short cyclical obsolescence of technology presents significant challenges for researchers and practitioners alike.

Knowledge of the IT adoption phenomenon for firms has also been limited to representative snapshots in time (Carter et al., 2001; Hameed et al., 2012) and, for this 
reason, it should ideally be regularly revisited by researchers in that subsequent technological waves could very well influence organizational processes and resource norms. Behavioral attributes driving technology adoption tend to shift in relation to major technology movements, thereby requiring constant reassessments of the core attributes of the various models (Bhattacharjee and Ramkumar, 2004). Furthermore, research encompassing the strategic and tactical value of IT to the organization is constantly evolving and often resists alignment with the previously researched characteristics of various models (Agarwal and Prasad, 1998).

The rapidly evolving nature of digital adoption will require some retooling of existing models especially as it relates to the transformation of a traditionally functional technology to that of a technology which is critical for digital adoption (Liu et al., 2011). The business digitization paradigm sets forth new requirements to explain how newly available technology is not only driving business process realignments, but contributing heavily to new ways to market agility, productivity, and profitability (Resca et al., 2013). DX, on the other hand, is a relatively new concept which promotes the core data network to support connected and disruptive applications which enable and support new agile business processes (Oosterhout, et al., 2006). Research in this area is extremely sparse because the data network has always been an afterthought in overall DX strategy as an historically functional technology (Chalons and Dufft, 2017). The data network is predominantly viewed as a purely operational technology where the crucial benchmark of its performance and usefulness within most enterprises is to deliver connectivity within expected performance tolerances. However, this does not reflect its ability to support 
emergent business processes, applications, end user requirements, or the business mission, which together constitute the core of successful DX project outcomes.

Since the beginning of computing, the data network has received little academic notice. This is perhaps explainable if the data network is, indeed, merely a technology that serves as an easily scalable infrastructure. In the case of DX, however, this assumption is likely invalid. DX is reliant on a robust and resilient data network to connect data centers, cloud, applications, business units, Internet of Things, etc. and it is not clear whether existing models and architectures can capture the functional-to-strategic realignment of data networks (Bharadwaj et al., 2013) nor its ability to successfully support various levels of digital adoption.

Where functional properties certainly do lay out the primary desirable characteristics of a data network, properties such as resiliency and reliability, organizational reliance on the data network to support the increasingly complex and critical requirements of DX call for new strategic adoption relationships not previously studied or understood. DX is thus driving a new need to understand the adoption/consumption rationale as it relates to the strategic organizational directives and goals.

\section{Purpose of this Study}

Current functional trends in data networking do not envision a radical differentiation from basic connectivity. Legacy network speeds, 10/100 Mbps, have been replaced by 1, 10, 40, and 100 Gbps Ethernet, and, wireless network standards, 802.11a, b, and, g, which offered wireless connectivity up to $450 \mathrm{mbps}$, have been replaced by WiFi standard protocols such as 802.11ac and 802.11ax (Wi-Fi 6), which can offer 
connection speeds greater than $2.5 \mathrm{Gbps}$. However, these advances in wired and wireless technologies are merely functional regarding typical organizational requirements, since most end-user applications do not require connection speeds at these levels, thus there is no strategic advantage. As a result, there are significant diminishing returns in that data networking equipment is still a commodity when applied in the traditional functional context (Carr, 2003).

Although the basic structure of the data network has remained relatively static, disruptive and radical innovation in the form of network automation, the introduction of centralized orchestration, artificial intelligence, machine learning, offer new capabilities for dynamically supporting the needs of the numerous applications deployed within the organization.

Artificial intelligence, for example, is driving network automation which can eliminate the main cause of $90 \%$ of data network issues, i.e., the network engineer. Modern networks are now capable of being built to specific intent (Beshley et al, 2019) rather than the generic one-size-fits-all posture of the past. Data network capabilities have shifted from only supporting their own weight (despite increased transport speeds and resiliency algorithms) to intelligently supporting the complex application architecture and underlying strategic business intent and requirements which are integral to digital transformation.

Bharadwaj et al. (2013) note that over the previous 30 years, Information Technology (IT) has often been viewed as only a functional resource that must support the firm's strategic initiatives, as Carr (2003) famously demonstrates in his HBR article. Moreover, if IT as a whole has heretofore only been viewed as a functional resource, then 
the data network has historically occupied the very bottom of this category, which is in line with the data network often being viewed only as a cost center with little potential to support profitability (Bharadwaj, et al., 2013).

This functional characterization has carried forward to our own day even though the data network must support the weight of exponential increases in data generation and consumption due to DX. The greater the level of digital transformation, the greater the amount of data generated (Hess et al., 2016); therefore, it follows that there is a greater the reliance on the data network to transport that data. Considering this scenario, it is unclear to what level current consumption/adoption decisions have shifted, if at all, to include strategic decision making as it relates to the current capabilities of the data network.

It is also unclear whether there has been shift of data networking procurement behaviors to ensure that data network technology is consumed and adopted with a deeper understanding of short- and long-term digital priorities and how the data network will strategically support them. Do the antecedents of IT adoption change over time and are the antecedents of technology consumption and adoption the same as the antecedents of usage (Karahanna et al., 1999)?

Additionally, there is the question of the relationship between adoption $(A)$ of data network equipment and consumption $(C)$ of $\mathrm{DX}$ technologies within the organization. A best-case scenario would suggest that $A$ would lead to a high ratio of $C$, which would indicate that strategic procurements lead to the higher DX consumption within the orgainization, a causal attribution that would indicate a positive relationship. 
Conversely, the lower the ratio of $A$-to- $C$, the greater the need to explain the functional-to-strategic divergence phenomena. Because research on this relationship remains extremely limited, there is a great deal of interest in research with a focus on execution-level processes (Tarafdar and Qrunfleh, 2010). Ultimately, this research reviews the relationships of adoption drivers of data network technologies specifically aligned to support digital transformation. Within this guise the research explores four core research questions:

(1) In what respect does DX necessitate that firms transform their procurement drivers of the data network from tactically aligned to strategically aligned?

(2) How are IT investments in the data network related to digital transformation project outcomes?

(3) How has DX caused data network adoption and consumption decisions to evolve?

(4) How are DX outcomes measured and are current models sufficient for describing DX project outcomes with the required levels of precision?

Because the preponderance of the body of related research frames IT investment as a functional operation (Bakos and Treacy, 1986; Henderson and Venkatraman, 1993; Drnevich and Croson, 2013), the current research will explore the relationship of the data network as it relates to various levels of DX outcomes. 


\section{CHAPTER 2}

\section{REVIEW OF LITERATURE}

Introduction

Despite voluminous literature generated on the phenomena of technology adoption, there are still significant gaps, especially in relation to process-level dynamic relationships and their direct effects on technology adoption outcomes (Tallon, 2007). Because technology is constantly evolving, the adoption behavior needs to adapt in relation to the available technology trends at a given point in time (Chiyangwa and Alexander, 2016). Therefore, metrics such as perceived usefulness and other variables in the technology adoption models are moving targets which must continuously be observed as technology evolves (Chiyangwa and Alexander, 2016). When one links together constantly evolving technology, business requirements, calls for more process-level research, and the ubiquitous nature of technology as an organizational process enabler, scholars must frequently revisit the available body of knowledge and the models which they support (Tallon, 2007).

Existing quantitative research has been invaluable in establishing causality in technology adoption and consumption models; however, the qualitative approach proposed in this research is an exploratory step in researching a relatively new phenomenon, e.g., digital transformation. As a result, DX's constant evolution presents an opportunity for exploratory work guiding deeper quantitative study. Because the technology focus of this research is not software application specific, as with nearly all current information technology adoption models, it is difficult to capture the behavioral 
aspects of the adoption action without the depth of observation afforded by a qualitative approach. Therefore, the case study research in this paper will contribute in the following ways:

1. Define the properties of strategically and tactically aligned data networks.

2. Directly observe the effect of strategically aligned versus tactically aligned data network on digital transformation projects; and

3. Formulate a method of precisely measuring the resultant DX outcomes.

It is well understood that technology is procured and deployed either for functional or strategic reasons (Henderson and Venkatraman, 1999), and the data network has historically been seen as a purely functional technology, with connectivity to other network devices and sites being its primary function. Functional data network procurement and deployments update aging equipment and provide for data connectivity and price is a key determinant for procurement. The novelty of new technology-tobusines integrations cause many deployments to be inward facing, in that stakeholders are mainly siloed as technologists and often lack the cross functional integration required to scope the network to support other technologies or business functions (Majchrzak et al., 2012) Therefore, the network is only deployed to successfully support the function of the data network and other directly related functional IT units, such as security or voice over IP. Any short- or long-term business benefits of a functional network have therefore been largely tangential or coincidental (Tarafdar and Qrunfleh, 2009).

Conversely, strategically aligned data network deployments are 'outward' facing in their procurement and deployment rationale; therefore, stakeholders for strategically aligned data network projects include both business and technology leaders. And, although the function of the network remains the same, the resultant impact on the business and the data network's ability to support both the short and long-term business 
mission, as defined by key business stakeholders, are key strategic issues, especially when one considers DX.

Information Technology Investments and Performance Outcomes

At its core, this paper studies the relationship between investments in IT, specifically the data network, and the resultant outcomes realized which are specific to levels of firmwide digitally transformative technology adoption. We start with IT investments and performance outcomes. Whereas the overarching concepts of technology investments-to-performance outcome relationships have been studied extensively, IT investment concepts covered in the literature thus far still lack conclusive research focused on purchase motivations, processes, and business outcome realization outside of financial and productivity benefits (Sriram and Stump, 2003). This dearth of noneconomic research matches the mostly organizational focus of IT investments which stress return on investment (ROI) in terms of finances and productivity. Therefore, if IT investments are only measured and researched in those terms, then is there an entire relationship dynamic being overlooked? Sriram and Stump (2003) attempted to fill this research gap by building an integrated model which also adds purchasing, and a topdown organizational conceptual model to the mix of desirable outcomes.

Despite the traditional view that optimal investments in IT should be translatable into performance and productivity, Sriram and Stump (2003) identified a productivity paradox. This paradox resulted in increased IT investment but does not lead to a consistent increase in productivity. We adopt this concept in the subsequent case study research when we observe whether specific strategic alignments precede desirable organizational outcomes. 
Key drivers of this paradox include management's failure to leverage IT's potential, ineffective implementation, incomplete measures of performance, and the presence of a time lag between IT investment and performance effects (Sriram and Stump, 2003). To help to resolve this paradox, Sriram and Stump (2003) argued that it is important for firms to recognize the multidimensional nature of IT investments to drive internal and external tactical and strategic initiatives while also constantly measuring IT alignment within the firm to ensure the right mix, and fit, at various stages of design and deployment. They conclude that there is a direct correlation between the IT investment, social aspects of the organization, and the successful realization of business outcomes (Sriram and Stump, 2003). As such, Sriram and Stump's (2003) research establishes that much deeper organizational interaction and dynamics are key to achieving optimal outcomes from IT investments through strategic alignment of the IT procurement. However, to gain a deeper understanding of how the literature views key mechanisms for deriving optimal IT investments, we next turn to Davern and Kauffman (2000) and their research into deriving optimal value from IT investments.

Deriving Value from IT Investments w/r/t Functional Technologies As developed by Sriram and Stump (2003), an understanding of the technology value-conversion is vital because it provides a mechanism for aligning actions with the appropriation of value from a given information technology investment (Davern and Kauffman, 2000). Justifying IT investments is always a challenge especially when it pertains to functionally aligned technologies. For instance, why should a legacy phone system be upgraded? One picks up the phone and there is a dial-tone. One presses the buttons and after a few seconds the intended party answers. The connection is relatively 
clear and there is a resounding "Hello," Such legacy phone systems are conveniently connected to vendors and customers by the telephone companies and they perform with consistent reliably indicative of the established, historic, public, switched telephone network. If one were to try to divine the appropriation of value for the replacement of a legacy phone system merely as a function of a new dial-tone, then the value vis-à-vis the current phone system would probably be close to zero.

Conversely, if the replacement of the legacy phone system is seen as an integral component of digital transformation, the value conversion is much more apparent. The system can offer productivity enhancements and it can introduce collaborative capabilities, both of which can provide new capabilities for the firm. A video feature in the replacement system that also permits recording (and thus sharing), for example, could transform the way collaboration takes place. The building blocks of value for appropriation of the technology become much less opaque and a quantifiable business case can, in fact, be made, with an appropriate calculation of value to the organization.

If, however, the underlying data infrastructure was not considered to be key to supporting the generation of new data and connectivity between data and telecom systems, then the expected value from the IT investments might not be met. The needs of video, recording, and sharing capabilities in the above example could easily overwhelm the network and breakdowns could be commonplace or even, fatal. In such a case, only viewing the data network functionally would be antithetical to the ultimate goals of the organization,

Part of this underlying problem is that many managers do not see traditionally functional technologies as evolving into strategic technologies in the current era of digital 
transformation (Davern and Kauffman, 2000). Davern and Kauffman (2000) found that many senior managers still have trouble with the value conversion process and accepting the appropriate conversion methodology as one that leads to a solid understanding of the potential value of the technology investment. They also found that it is only when potential value is measured against actual value after the implementation that $\rightarrow$ the real value of the chosen technology becomes clear. Thus, Davern and Kauffman (2000) offer a new theoretical framework based on Potential Value $\rightarrow$ Conversion Contingencies $\rightarrow$ Realized Value, an explicit extension to the Locus of Value Model (Kauffman and Weill, 1989). This model identifies value both top-down and bottom-up. It begins with market value down to the individual user and vice-versa. The intermediate values are business process, workgroup, and firm. Any question of applying strategic business outcome to an IT purchase should be inclusive of at least the "Locus of Value" as an ex-ante and ex-post consideration.

Without a doubt there are opportunities for further research with the requirements for IT purchases being better aligned with complementary technologies (Davern and Kaufman, 2000). In the example above of investing in a new phone system, the IT investment must be valued not only with regard to the basic technology required but also in the complementary technologies and processes which will, ex-post, be positively (or negatively) influential.

Given that IT investment is a generic process required of all IT organizations, we turn our attention next to detailing strategic vis-à-vis functional IT purchase drivers because the alignment of the purchase will have a direct impact on short and long term organizational digital capabilities and adoption levels (Drnevich and Croson, 2013). 
Identifying and Classifying Functional and Strategic Technologies

Drnevich and Croson (2013) researched the functional-to-strategic transformation requirement of IT operations by reviewing the available literature and offering further discussion on the components of IT. As firms struggle to transform information technology from a largely functional area of operation to a strategic tool for enabling meaningful business outcomes, IT investments are driving new and unfamiliar expectations of expanded business outcomes. IT investments have been integral to operations at the functional-level of the firm, but they now play an increasingly substantial, and largely under-theorized, role in business-level strategy.

Drnevich and Croson (2013) depart from the status quo of the holistic, generalized description of information technology by deconstructing the IT resource. They identify IT resources as: (1) tangible IT infrastructure, (2) skilled human IT specialists and managers, (3) intangible knowledge-based assets (Bharadwaj 2000; Drnevich and Croson, 2013). Organizational investment is therefore being made in all three of these IT resource dimensions, indicating that there are possibilities of realizing business value from the IT investment in all three dimensions (Drnevich and Croson). While Drnevich and Croson (2013) expand the notion of information technology to include the human element, their research does not extend the purely technological element or attempt to define specific technologies as either strategic or functional. Nor does their research provide examples of technologies which are critical to the emerging functional-to-strategic technology evolution that lies at the heart of DX.

In a previous attempt to decompose the IT artifact, Weill et al. (2002) suggested that managers at top-performing firms have a highly developed sense of business 
requirements and initiatives before they determine what information technology mix and fit is required to achieve their identified goals. Additionally, they found that high performing firms have a more mature cross-functional communications relationship between business units in place so that there is a deeper appreciation of the need to apply shared, versus individually customized, software and hardware assets. Good IT fit, as a way of measuring the correct level of IT spending, is vital, they argue, to prevent the waste of IT in either over- or under-investment.

Weill et al. (2002) used a quantitative research approach with surveys for data collection, sampling 180 business initiatives at eighty-nine enterprise organizations. Their research shows that paying attention to the correct balance can result in a dip in short term profitability (due to the required capital expenditures) but faster times to market, higher growth rates, and higher sales of new products (Weill et al., 2002).

Weill et al. (2002) then moved their research to the next logical step which is to provide high-level identification of technologies at each layer of their model of strategic alignment. Technology-to-business alignment was deconstructed in their work and categorized into ten business clusters: channel management, security, communications, data-management, application-infrastructure, IT-facilities management, IT-management, IT-architecture and standards, IT-education, and research and development. Irrespective of the category or the high-level technology applied, their research findings indicate that a robust and customized, high-capacity infrastructure must exist at the core (Weill et al., 2002).

Other researchers have delved into the question of alignment, but from the tactical perspective and we review their work next. As noted above, scholars looking at 
alignment from the higher strategic levels typically talk about the "IT" investment as a whole and do not consider elements within the infrastructure, especially the hardware infrastructure, as being strategic in and of themselves.

\section{Organization and Information Technology Strategic Alignment}

The alignment of strategic business outcomes-to-information technology procurement is vital to realizing optimal return on investment from IT investments (Wu, et al., 2015). Despite the necessity of understanding and continually measuring the fit between business and information technology, academics and practitioners continue to be challenged by the complexities of conceptualization and measurement (Tallon, 2007). Tallon explored this continual search to understand business-to-information technology synergies. Tallon remarks that there is a supposition that IT and business alignments are strongest where there is a well-defined focus on critical process-level requirements. However strategic alignment varies so widely between firms that even firms of similar size and in similar market verticals will utilize similar technology to achieve totally dissimilar business outcomes.

Because IT alignment-to-strategic fit varies so widely across organizations, Tallon posed two research questions. First, do differences in strategy or strategic foci translate into differences in alignment at the process level? Second, is the link between alignment and firm performance that has been observed at the firm level also valid at the process level? These questions are particularly relevant, as technology leaders within firm organizations are continually being tasked with extracting more value from IT purchases. Understanding these outcomes is also a core driver for the proposed research outlined in 
this paper. Tallon uses the value discipline topology by Treacy and Wiersema (1993) as the key to answering these questions.

Tallon's (2007) conceptual model has business strategy and IT strategy as separate antecedents which combine to form strategic alignment. Strategic alignment subsequently leads to IT business value. Specifically, Tallon conducted a field study analysis of 1,600 firms, a random subset of 2,826 publicly traded firms between $\$ 100$ million and $\$ 3$ billion across a wide variety of market verticals. Focal respondents were senior business and senior IT leadership who were given surveys to measure IT and business alignment. T-tests measured relationships at a .95 confidence interval with IT business value being measured via a 7-point Likert scale anchored from low to high IT impact. Validation of the construct was via a confirmatory factor analysis of business strategy, IT use, and IT value responses. Tallon also engaged industry experts to review the survey data. These industry practitioners were pulled from a cross section of MIS disciplines and included IT operation leadership, executives, and consultants in addition to a group of IT focused academics. Tallon's findings indicate that the relationship of the organizational resource and process alignment to information technology strategy is overall positive and significant.

While Tallon provided valuable incremental knowledge to understanding business-to-IT alignment, there are still many research gaps, offering opportunity for subsequent study. First, the study makes no attempt to deconstruct the information technology variable. IT is treated as a holistic and all-encompassing entity that must be aligned to the business. Because IT is operationally dispersed among various composite sections, such as security, applications, infrastructure, communications, etc., there is no 
indication of the individual process and resource level alignment at the various firms and if these alignment variances (if any) were statistically significant.

Second, Tallon indicated that his approach to explaining business to technology alignment is open to interpretation. If this is the case, then ambiguity in the body of literature might have been better served by investigating the phenomena by casting a wider net, e.g. using multiple respondents to confirm the relationships.

In contrast, Ullah and Lai (2013) took a technologist approach to the same set of relationships. Their paper is structured as a review of the current knowledgebase pertaining to the research area of IT-to-business alignment. The paper is valuable because it proposes many comprehensive research questions in addition to a summary of findings from the existing literature. Their findings argue that bridging the gap between business and IT is difficult, not only due to differences in organizational objectives, structures, and culture, but also because of the constantly shifting business requirements which must align with constantly shifting technologies (see also Luftman et al., 1993, 1999; Reich and Benbasat 1996; Sauer and Yetton, 1997; Ullah and Lai, 2013). The challenges identified by Ullah and Lai are of particular interest to the current research in that DX technologies are a rapidly moving target.

Ullah and Lai also reify the fit requirement researched by Tallon (2007) as well as Henderson and Venkatraman (1993), who state that alignment between business and IT is the degree of fit and integration between business strategy, IT strategy, business infrastructure, and IT infrastructure. To study and test these alignment synergies, Ullah and Lai (2013) focused on literature from highly IT-centric journals such as JIT, MISQE, $M I S Q$, JCIS, and JMIS. Systematic review of this literature was conducted over a three- 
year period of 2009 through 2011. The completion of the review drove ten research questions. Of especial interest is RQ\#10, which delves deeper into specific technologies as a subset of a larger technological landscape. However, Ullah and Lai only refer specifically to software development challenges to business alignment. While software development faces unique challenges, this technology is only tangentially related to the data network. As such, the strategic transformation of information technology hardware, such as the data network infrastructure, is again omitted in their work. Ullah and Lai do offer a comprehensive review of the current state-of-the art in the business-to-IT alignment area of research. Research questions and findings from the literature provide helpful insights which researchers can utilize to not only understand what is available from the current literature but also offer avenues which lead to opportunities for new research.

Having established that understanding IT alignment is still at issue in the current IT literature, we now turn to how the introduction of contemporary technologies, business trends, and their requisite alignment challenges existing technology adoption research.

The Fourth Industrial Revolution, Digital Transformation and Related Research Gaps

Thus far, the existing body of research has covered traditional firm-level technology alignment and adoption. However, it is increasingly being found that traditional explanations of adoption technology in firms labor to explain fit (Liu et al., 2011). As such, the contemporary, disruptive organizational phenomenon, collectively referred to as the fourth industrial revolution, is causing researchers to reassess previously researched technology-to-business relationships (Murdoch and Fichter, 2017). 
The fourth industrial revolution was first described by Schwab (2015) as being led by new technologies such as mobile supercomputing, artificial intelligence and machine learning, cloud, and self-driving cars. While these nascent technologies introduce new organizational challenges and opportunities on multiple levels, a common thread across all the technologies is their inherent reliance on inter-connectivity (Miller, 2015, Xu et al., 2018), i.e., the data network, in order to operate at all.

In addition to the introduction of connected technologies, Demirkan et al. (2017) emphasize the importance of digital transformation as a key aspect of the fourth industrial revolution, as noted above in their definition of DX. Demirkan et al., identify this transformation is occurring not just at the enterprise level, but at the very core of society. Therefore, digitalization effectually allows enterprises to respond to the customer base more quickly while at the same time offering the consumer a greater selection of products and services delivered through the course of disruptive and radical innovation.

Ultimately, Demirkan et al. assert that enterprises must identify digitalization objectives at all levels to successfully support the transformation. While technology is important, firms must build and integrate the required skills within the firm to support these new technologies, processes, and services. Although firms will likely not have all the resources required before or even after their digital transformations, investments in personnel and expansion of absorptive capacity of external knowledge are critical to success.

Demirkan et al also suggest that organizations must focus on building a solid digitalization roadmap and framework, definitions of business outcomes, capabilities, and critical supportive technologies as well as ensuring required skillsets are accessible. 
However, they maintain that appropriate human and technical resources to support digital transformation being in the right place, at the right time, is not always well understood. The path to successful digital transformation differs for each firm and empirical academic research in these areas often require the depth and breadth of inductive qualitative methodologies to accurately measure the core of the phenomenon as it affects each firm. To this end, academic research in this area remains relatively light and skewed toward practitioner research. Acknowledging this gap in the research, Demirkan et al. urge doctoral students to further study digital innovation and transformation.

Building on the critical internal skillset requirements indicated by Demirkan at al. (2017), Kranz et al. (2016) researched how expanding absorptive capacity is required to support business model changes. Kranz et al. found that there is a gap in empirical understanding of how digital transformation affects the enterprise in addition to how it should be mitigated, supported, and managed. Their multiple case study approach with grounded theory data collection offers a framework that builds upon prior research in absorptive capacity. The study focuses on absorptive capacity and ambidexterity in the assimilation of a disruptive innovation; cloud-computing. Kranz et al. assert that one of the greatest challenges in adopting disruptive technology is the requirement for fundamental change in the dominant logic which supports current process methodologies. To this end, their research shows that absorptive capacity is critical for drawing in new knowledge which lessens the impact of change while ambidexterity provides the capability for combining existing and newly gained knowledge into the enterprise.

As shown in Table 1, there is a growing body of research on the knowledge requirements for successful digital transformation. However, despite this growing body 
of research there is still a gap regarding "the how and why technical infrastructures evolve over time" (Henfridsson and Bygstad, 2013, p. 907) as well as how the evolution of technology within the enterprise increases the complexities of the relationship between business and IT units. They performed a multiple case study, surveying 41 firms to identify generative mechanisms of digital infrastructure and their transformation within the organization over time. This study therefore combines the need to understand both the human and technical elements of technology and digital transformation initiatives. Their view of the data network is that it goes beyond mere functionality in spite of the fact that they refer to the digital infrastructure as "the collection of technological and human components, networks, systems, and the processes that contribute to the functioning of an information system" (Henfridsson and Bygstad, 2013, p. 908). Their choice of the word "functioning" here is perhaps unfortunate, but their essential message is that the data infrastructure can certainly involve fundamentally strategic decisions.

Given these insights into digital transformation, why do the established framings of organizational change not apply (Liu et al., 2011)? Among the most dominant paradigms dealing with how organizations change with the times have been the resourcefocused theories. So as compelling as the research on digital transformation has been thus far in favor of ensuring human and technical resource alignment, noticeably absent from the research has been any substantive mention of Dynamic Capabilities Theory (DCT) and the Resource Based View (RBV) of the firm (Pisano and Shuen, 1997). Arguably this is because digital transformation renders conventional technology adoption constructs, such as those in DCT and RBV, obsolete and insufficient for describing the digital transformation phenomena. (Liu et al., 2011). Notably, Liu et al.'s case study supports 
the application of resource fit by Harmancioglu et al. (2009), a study providing a more suitable construct combining RBV constructs and strategic fit.

Because digital transformation often drives complex and radical change over a relatively short period of time the dynamics of this transformation are often overlooked or occluded by viewing the firm as a heterogenous entity (Liu et al., 2011). Liu et al. found that competitive advantages arise from driving more efficient use of resources rather than just having the best resources. As such, internal and external resource strategic alignment, i.e., fit, is key. However, the lack of fit, or the lack of understanding of the appropriate fit, is still ambiguous relevant to the successful adoption of digital initiatives.

\section{Table 1}

Sampling of Previous Literature on Digital Transformation

\begin{tabular}{|c|c|c|}
\hline Author (date) & Title & $\begin{array}{l}\text { Key Findings } \\
\end{array}$ \\
\hline $\begin{array}{l}\text { Delmond et al. } \\
\text { (2016) }\end{array}$ & $\begin{array}{l}\text { "How Information Systems } \\
\text { Enable Digital } \\
\text { Transformation: A Focus } \\
\text { on Business Models and } \\
\text { Value Co-production" }\end{array}$ & $\begin{array}{l}\text { Strong partnership between IT and } \\
\text { business is a key factor leading to } \\
\text { organizational agility. } \\
\text { Organizations must pay close attention to } \\
\text { the information technology infrastructure. }\end{array}$ \\
\hline $\begin{array}{l}\text { Demirkan et al. } \\
\text { (2016) }\end{array}$ & $\begin{array}{l}\text { "Digital Innovation and } \\
\text { Strategic Transformation." }\end{array}$ & $\begin{array}{l}\text { It is critical that enterprises identify } \\
\text { digitalization objectives at all levels to } \\
\text { successfully support the transformation. } \\
\text { Academic research in strategic IT use in } \\
\text { digital transformation is relatively light } \\
\text { and skewed toward practitioner research. }\end{array}$ \\
\hline $\begin{array}{l}\text { Henfridsson and } \\
\text { Bygstad (2013) }\end{array}$ & $\begin{array}{l}\text { "The Generative } \\
\text { Mechanisms of Digital } \\
\text { Infrastructure Evolution" }\end{array}$ & $\begin{array}{l}\text { Adoption, innovation, and scaling are } \\
\text { three generative mechanisms within the } \\
\text { construct of contingent causality. } \\
\text { Digitalization increases the complexities } \\
\text { of the relationship between business and } \\
\text { IT units. }\end{array}$ \\
\hline
\end{tabular}




\begin{tabular}{|c|c|c|}
\hline \multicolumn{3}{|c|}{$\begin{array}{l}\text { Table } 1 \text { Continued } \\
\text { Sampling of Previous Literature on Digital Transformation }\end{array}$} \\
\hline $\begin{array}{l}\text { Kranz et al. } \\
(2016)\end{array}$ & $\begin{array}{l}\text { "Understanding the } \\
\text { Influence of Absorptive } \\
\text { Capacity and } \\
\text { Ambidexterity on the } \\
\text { Process of Business Model } \\
\text { Change-The Case of On- } \\
\text { Premises and Cloud- } \\
\text { Computing Software" }\end{array}$ & $\begin{array}{l}\text { Expanding absorptive capacity is } \\
\text { required to support business model } \\
\text { changes. } \\
\text { Requirement for fundamental change in } \\
\text { the dominant logic which supports } \\
\text { current process methodologies. }\end{array}$ \\
\hline Liu et al. (2011) & $\begin{array}{l}\text { "Resource Fit in Digital } \\
\text { Transformation: Lessons } \\
\text { Learned from the CBC } \\
\text { Bank Global e-banking } \\
\text { project. " }\end{array}$ & $\begin{array}{l}\text { Digitalization is rendering conventional } \\
\text { constructs, such as RBV, insufficient for } \\
\text { describing the digital transformation } \\
\text { phenomena. } \\
\text { Lack of understanding of appropriate fit } \\
\text { remains a key issue to the successful } \\
\text { adoption of digital initiatives. }\end{array}$ \\
\hline $\begin{array}{l}\text { Resca et al. } \\
(2013)\end{array}$ & $\begin{array}{l}\text { "Digital Platforms as } \\
\text { Sources for Organizational } \\
\text { and } \\
\text { Strategic Transformation: A } \\
\text { Case Study of the Midblue } \\
\text { Project" }\end{array}$ & $\begin{array}{l}\text { IT architectures and operational } \\
\text { paradigms offer flexibility and scalability } \\
\text { that surpass traditional transformative } \\
\text { capabilities. } \\
\text { Successful digital transformation and } \\
\text { adoption requires a tightly integrated } \\
\text { approach at all organizational levels. }\end{array}$ \\
\hline
\end{tabular}

In summary, a gap in research exists despite the extensive study of traditional organizational structure and information technology because digital IT architectures and operational paradigms offer flexibility and scalability that surpass traditional transformative capabilities (Resca et al., 2013). To expand extant frameworks to support expanded digital capabilities, Pisano and Shuen (1997) present an integrated framework for IT enabled organizational transformation which was validated through their case study research. In their framework, IT architecture, organizational structure, and business strategy are perspectives (a.k.a. platforms) which are supported by key conceptual properties, all of which must be considered within the scope of the framework. They found that the three platforms of the framework are intertwined and inseparable, inexorably requiring an integrated approach to digital transformation. 
This integrated framework requirement for supporting digital transformation is also argued in research by Delmond et al. (2016). In their case study, the key capabilities of the information system to support new business models are defined as: tangible resources in the form of IT infrastructure and human resources in the form of the skills of technical and managerial resources. Additionally, there are intangible resources such as knowledge, customers, and synergies (Bharadwaj, 2000; Delmond et al., 2016).

Furthermore, Delmond et al.'s work indicates that a strong partnership between IT and business is a key factor to organizational agility, leading to three overarching principles: (1) organizations must pay close attention to the information technology infrastructure; (2) organizations must include external partners as a part of their IT strategy; and (3) organizations cannot manage complex information technology environments through an organizationally siloed approach. Firms must include external technical resources and be aware of absorptive capacity and ambidexterity capabilities.

Finally, in assessing the critical gaps in the literature, while contemporary research is providing new frameworks and reassessments of the role of IT as a strategic and core property of the enterprise mission, Delmond et al. (2016) say that there is no one-size fits all strategy that is able to address digital transformation requirements on a large scale (For instance, cloud computing is continuing to disrupt traditional on-premises technologies and services; however, cloud adoption strategies still vary widely across organizations (Kenney et al., 2015).

Organizations adopt technology at different rates and for different reasons, but some key concepts transcend organizational or industry verticals (Parente et al., 1994). Successful digital transformation requires that business and IT work closely to clearly 
define resources, scope, and strategy. (Gurbaxani et al., 2019). A failure in part of the operating framework can have negative impacts on the success of the transformation. Digital transformation is complex and has wide ranging ramifications for the enterprise therefore research in the area will struggle to stay current as incremental, radical, and disruptive innovations cause rapid change to the landscape (Jöhnk et al., 2020).

Having established that DX is characterized by upheaval and that there are major gaps in research as it pertains to the strategic adoption of digital transformation technologies, the natural question is: What next? Thus far we have only touched upon the technology adoption frameworks which are at the core of how the core resources of the firm, the end users, make the decision to adopt technology. Therefore, we next briefly review technology adoption models to better understand their capability for explaining DX technology adoption at a greater depth and if these models can be used to support this research.

\section{Technology Models and Frameworks in a Transforming Landscape}

To better understand patterns and motivations for technology adoption and consumption, significant prior research has been directed at studying the behavioral patterns of end users and the methods by which technology disseminates within a target population. Modeling these behavioral patterns is particularly challenging as technologies which have been historically transparent to the end user, such as the data network, are now becoming essential to supporting end user technology; likewise, technologies which were once considered to be disruptive have themselves often been disrupted.

The data network is a key example of a historically transparent technology which has become an essential for many of the latest disruptive technologies. For instance, IoT, 
automotive telematics, artificial intelligence, machine learning, and big data - to name only a few - all fail in the absence of an adequately supportive and specifically architected data network.

There are practitioner solutions that reify this line of thought. Strategic technology alignment road mapping, STAR®, provides a series of structured frameworks for aligning technology purchases with business outcomes, providing for more deeply integrated applications, especially when it is applied to DX. Within such frameworks are technology data collection, project creation, and assessment phases (Gindy et al., 2007). This framework currently measures the economics, technical alignment, and synergy of the technology proposal as it pertains to $\mathrm{R}$ and $\mathrm{D}$ investments.

Yet, while useful in many ways, this framework does not attempt to explain how the application of STAR $®$ would affect the application of functional technologies in a non-R and D-based organizational setting. Furthermore, because the framework is needsdriven, it assumes that the organization understands its technology needs and the structure required to support those needs. STAR ${ }^{\circledR}$ does not explain how a technology like the data network, which has historically been largely ignored and treated as a cost-center, would be included in strategic determinations.

Furthermore, the current literature does not sufficiently capture the use of traditionally functional technologies in tactical and strategic roles within the enterprise and to what extent this use can be weighed in technology purchases (Goerzig and Bauernhansl, 2018). This lack of understanding is especially evident as it relates to data network equipment, a traditionally functional technology, where lines between functional value and strategic value are today being blurred due to newly available technical 
functionalities. Instead, the research models outlined in Table 2 focus on inherently strategic or tactical applications such as e-commerce, ERP systems, various corporate software applications, and websites.

From an organizational standpoint, technology adoption and alignment research need to be studied in a broader context because business investments in technology are not only individual enablers but contribute to the broader goals of the firm (Agarwal and Lucas, 2005). However, despite extensive research, failed IT projects continue to lead to a high ratio of irrelevant applications deployed within organizations (Tarafdar and Qrunfleh, 2010).

\begin{tabular}{|c|c|c|c|c|}
\hline \multicolumn{5}{|c|}{$\begin{array}{l}\text { Table } 2 \\
\text { Selected Literature on Models of Strategic Technology Alignment / Adoption-to- } \\
\text { Business Requirements }\end{array}$} \\
\hline Technology & Research Variables & Methodology & Data Collection & Author \\
\hline $\begin{array}{l}\text { Various } \\
\text { adoption } \\
\text { strategic } \\
\text { enterprise } \\
\text { technologies }\end{array}$ & $\begin{array}{l}\text { Initiation, adoption, } \\
\text { implementation, } \\
\text { infusion, integration, } \\
\text { assimilation, usage }\end{array}$ & $\begin{array}{l}\text { Analysis of existing } \\
\text { models: DOI, } \\
\text { TAM, TPB, TRA, } \\
\text { TAM2, TOE, TTF, } \\
\text { UTAUT }^{1}\end{array}$ & $\begin{array}{l}\text { Exhaustive } \\
\text { literature review of } \\
151 \text { publications }\end{array}$ & $\begin{array}{l}\text { Hameed, } \\
\text { Counsell, } \\
\text { and Swift, } \\
2012\end{array}$ \\
\hline $\begin{array}{l}\text { Various } \\
\text { alignment } \\
\text { strategic } \\
\text { enterprise } \\
\text { technologies }\end{array}$ & $\begin{array}{l}\text { Business strategy } \rightarrow \\
\text { strategic alignment } \\
\text { process } \rightarrow \text { IT } \\
\text { business value } \\
\text { IT strategy } \rightarrow \\
\text { strategic alignment } \\
\text { process } \rightarrow \text { IT } \\
\text { business value }\end{array}$ & Field Study & $\begin{array}{l}\text { Surveys of } 1,600 \\
\text { randomly drawn } \\
\text { firms from a } \\
\text { population of } 2,826 \\
\text { firms having a } \\
\text { revenue of } \$ 100 \mathrm{~m} \text { - } \\
\text { \$3B. }\end{array}$ & $\begin{array}{l}\text { Tallon, } \\
2008\end{array}$ \\
\hline
\end{tabular}

\footnotetext{
${ }^{1}$ Diffusion of Innovation (DOI), Technology Acceptance Model (TAM), Theory of Planned Behavior (TPB), Theory of Reasonable Action (TRA), Technology Acceptance Model 2 (TAM2), Technology Organizational Environmental (TOE) Framework, Unified Theory of, Task Technology Fit (TTF), Acceptance and Use of Technology (UTAUT)
} 


\begin{tabular}{|c|c|c|c|c|}
\hline \multicolumn{5}{|c|}{$\begin{array}{l}\text { Table } 2 \text { Continued } \\
\text { Selected Literature on Models of Strategic Technology Alignment / Adoption-to- } \\
\text { Business Requirements }\end{array}$} \\
\hline $\begin{array}{l}\text { Various } \\
\text { alignment } \\
\text { strategic } \\
\text { enterprise } \\
\text { technologies }\end{array}$ & $\begin{array}{l}\text { Alignment of } \\
\text { business to IT } \\
\text { objectives }\end{array}$ & $\begin{array}{l}\text { Literature review } \\
\text { using Kitchenham } \\
\text { (2004) systematic } \\
\text { guidelines. }\end{array}$ & $\begin{array}{l}\text { Analysis of existing } \\
\text { literature using } \\
\text { multiple traditional } \\
\text { scholar sources } \\
\text { using keywords: } \\
\text { business and IT } \\
\text { alignment; IT and } \\
\text { business alignment }\end{array}$ & $\begin{array}{l}\text { Ullah and } \\
\text { Lai, } 2013\end{array}$ \\
\hline $\begin{array}{l}\text { Various } \\
\text { alignment } \\
\text { strategic } \\
\text { enterprise } \\
\text { technologies }\end{array}$ & $\begin{array}{l}\text { Alignment of } \\
\text { business to IT } \\
\text { objectives }\end{array}$ & $\begin{array}{l}\text { Detailed pattern } \\
\text { analysis of } \\
\text { collected data }\end{array}$ & $\begin{array}{l}\text { Analysis of } 4 \\
\text { studies inclusive of } \\
118 \text { businesses } \\
\text { from } 1990-2001 \text {. } \\
\text { In-person } \\
\text { Interviews and } \\
\text { surveys of }+90 \% \text { of } \\
\text { businesses from } \\
\text { study. } \\
\text { Supplemental } \\
\text { phone and email } \\
\text { data collection. }\end{array}$ & $\begin{array}{l}\text { Weill, } \\
\text { Subramani } \\
\text { and } \\
\text { Broadbent., } \\
2002\end{array}$ \\
\hline $\begin{array}{l}\text { Various } \\
\text { strategic } \\
\text { enterprise } \\
\text { technologies }\end{array}$ & $\begin{array}{l}\text { Competitive climate } \\
\text { quality assurance } \\
\text { programs } \\
\text { communications } \\
\text { frequency } \\
\text { quality of } \\
\text { relationships } \\
\text { cost } \\
\text { cycle times } \\
\text { process } \\
\text { improvements }\end{array}$ & Field study & $\begin{array}{l}\text { Sample size of } \\
2000 \text { procurement } \\
\text { professionals at } 277 \\
\text { firms with average } \\
\text { number of } \\
\text { employees: } 1407 \\
\text { and average } \\
\text { purchasing volume: } \\
\$ 169.3 \mathrm{~mm} \text {. }\end{array}$ & $\begin{array}{l}\text { Sriram and } \\
\text { Stump, } \\
2003\end{array}$ \\
\hline
\end{tabular}




\begin{tabular}{|c|c|c|c|c|}
\hline \multicolumn{5}{|c|}{$\begin{array}{l}\text { Table } 2 \text { Continued } \\
\text { Selected Literature on Models of Strategic Technology Alignment / Adoption-to- } \\
\text { Business Requirements }\end{array}$} \\
\hline $\begin{array}{l}\text { Various } \\
\text { strategic } \\
\text { enterprise } \\
\text { technologies } \\
\text { - software } \\
\text { focused }\end{array}$ & $\begin{array}{l}\text { Potential value } \rightarrow \\
\text { conversion } \\
\text { contingencies } \rightarrow \\
\text { realized value }\end{array}$ & Field study & $\begin{array}{l}\text { New framework } \\
\text { argument }\end{array}$ & $\begin{array}{l}\text { Davern and } \\
\text { Kauffman, } \\
2000\end{array}$ \\
\hline $\begin{array}{l}\text { Cloud } \\
\text { Computing }\end{array}$ & $\begin{array}{l}\text { Management } \\
\text { innovation } \rightarrow \\
\text { technology adoption }\end{array}$ & $\begin{array}{l}\text { Grounded theory } \\
\text { iteration }\end{array}$ & $\begin{array}{l}\text { Semi-structured } \\
\text { interviews, focus } \\
\text { group, field study } \\
\text { and observation, } \\
\text { searching internal } \\
\text { and external } \\
\text { archival data. }\end{array}$ & $\begin{array}{l}\text { Khanagha, } \\
\text { Volberda, } \\
\text { and Sidhu } \\
\text { et al., } 2013\end{array}$ \\
\hline $\begin{array}{l}\text { Database } \\
\text { and web } \\
\text { portal }\end{array}$ & $\begin{array}{l}\text { Drivers } \rightarrow \\
\text { (purchasing process } \\
\leftrightarrow \text { purchasing } \\
\text { organization) }\end{array}$ & $\begin{array}{l}\text { Data coded and } \\
\text { two-level cross- } \\
\text { case analysis }\end{array}$ & $\begin{array}{l}\text { Semi-structured } \\
\text { interviews and } \\
\text { secondary sources } \\
\text { between } 2007- \\
2010.12 \text { firms of } \\
\text { sizes varying from } \\
800 \text { thru } 80,000 \\
\text { employees. }\end{array}$ & $\begin{array}{l}\text { Luzzini, } \\
\text { Longoni, } \\
\text { and } \\
\text { Moretto et } \\
\text { al., } 2014\end{array}$ \\
\hline
\end{tabular}

This literature focuses heavily on why and how IT projects fail. Tarafdar and Qrunfleh (2010) show that up to $\$ 150$ billion in IT projects fail in the United States each year and $68 \%$ of stated IT projects do not fulfil the required objectives. Similarly, studies found that as many as $75 \%$ of new systems do not improve business processes or create any tangible financial benefit. These misspent resources result in over $\$ 130$ billion in wasted IT spend each year. Research also indicates that almost $50 \%$ of IT projects finish late and over budget (Tarafdar and Qrunfleh, 2010).

Tarafdar and Qrunfleh's research also uncovered that a major cause of such waste of time and money is the lack of alignment between business and the portfolio of IT applications used within the firm organization. This mismatch between business and IT operations is the real cost of lack of strategic IT-to-business alignment. However, unlike 
the conventional literature which studies technology-to-business alignment as a singular entity, they stratified the construct further into social, project, and outcome variables. The social aspect relates to the IT-to-business relationship at all relevant organizational levels. The project aspect relates to the selection and governance that contribute positively to the business. Finally, execution-focused alignment drives business outcomes which contributes to the larger corporate goals. Perhaps needless to say, their work, like that of the previous research reviewed, does not decompose IT technologies, and consider what the effects of lack of alignment might be in specific technologies, either traditional IT or DX IT.

While the foundational idea of strategic alignment for adoption of technology are certainly present in the current literature, research directly supporting the themes articulated in this paper is scant, making only an oblique argument for alignment. It is therefore the position of this paper that this critical research gap exists because of the lack of assessment of data network technologies specifically as an independent variable.

One could be forgiven if one were to posit that despite the gaps identified in this section that the existing models were sufficient, and that extrapolation would be sufficient for explaining the DX technology adoption phenomena. However, as we shall cover in the next section, we feel that this is not the case.

\section{Information Technology Adoption}

Expanding on the fact that there is already extensive research and literature dedicated to understanding how information technology has been adopted, academics and practitioners have found that there is still relatively limited understanding of the IT adoption phenomenon in organizations (Carter et al., 2001; Hameed et al., 2012). The 
scope of most of the literature is understanding decision making about IT investments to the point of a formal acquisition of the technologies (consumption) but there is very little examination of the time after the technology is consumed to gauge the level of pervasiveness of the technology within the firm over given time periods (Hameed et al., 2012). It is important to pause here and absorb this important point made by Hameed et al. The research is not only insufficient to explain IT adoption in an organizational setting, but post adoption measurements are also insufficient (Hameed et al, 2012).

Hameed et al. make strides to fill this gap by specifically targeting innovation dissemination from procurement to adoption via their literature review of the current state of research. Reviewing relevant literature from 1981 through 2012 and all the adoption models listed in Table 3, they discuss the available models, land attempt to understand how well these models capture the IT adoption phenomena. They also provide a cohesive model of their own to fill obvious gaps in the most relevant IT adoption and consumption models as they relate to organizations.

\begin{tabular}{|l|l|}
\hline \multicolumn{2}{|l|}{ Table 3} \\
Most Preeminent Information Technology Adoption Models (Hameed et al., 2012) \\
\hline \multicolumn{2}{|c|}{ Muthor } \\
\hline Theory of Reasoned Action (TRA) & \multicolumn{1}{c|}{ Fishbein and Ajzen, 1975 } \\
\hline Diffusion of Innovation Theory (DOI) & Rogers, 1983 \\
\hline Diffusion / Implementation Model & Kwon and Zmud, 1987 \\
\hline Social Cognitive Theory (SCT) & Bandura, 1989 \\
\hline Technology Acceptance Model (TAM) & Davis, 1989 \\
\hline Technology Organization and Environment (TOE) & Tornatzky and Fleischer, 1990 \\
\hline Theory of Planned Behavior (TPB) & Ajzen, 1991 \\
\hline Perceived Characteristics of Innovation (PCI) & Moore and Benbasat, 1991 \\
\hline Tri-Core Model & Swanson, 1994 \\
\hline Task-Technology Fit & Goodhue and Thompson, 1995 \\
\hline IT Innovation Adoption Research Model & Agarwal and Prasad, 1998 \\
\hline
\end{tabular}




\begin{tabular}{|c|c|}
\hline \multicolumn{2}{|c|}{$\begin{array}{l}\text { Table } 3 \text { Continued } \\
\text { Most Preeminent Information Technology Adoption Models (Hameed et al., 2012) }\end{array}$} \\
\hline Technology Acceptance Model 2 & Venkatesh and Davis, 2000 \\
\hline $\begin{array}{l}\text { Framework for Innovation Adoption and } \\
\text { Implementation }\end{array}$ & Gallivan, 2001 \\
\hline $\begin{array}{l}\text { Unified Theory of Acceptance and Use of } \\
\text { Technology (UTAUT) }\end{array}$ & Venkatesh et al., 2003 \\
\hline Technology Acceptance Model 3 & Venkatesh and Bala, 2008 \\
\hline
\end{tabular}

The criteria for the study were: (1) empirical studies of innovation adoption (2) innovation adoption specifically in firm organizations (3) dependent variables: initiation, adoption, implementation, infusion, integration, assimilation, usage, (4) organizational context (Hameed et al., 2012). It is well understood that there is no single model which explains IT adoption (Fichman and Carroll, 1999; Hameed et al., 2012); therefore, researchers in information technology adoption must use several IT adoption theories to explain behavioral phenomena both at the individual and organizational levels.

Hameed et al. provided valuable insight into all the major IT adoption models from DOI through UTAUT. Of great value is their identification of DOI, TAM, TRA and TPB as the most widely used models. Of these models, Rogers' DOI model (1983) is the most useful in that it relates directly to the study of IT adoption at the organizational level. This insight is a guidepost for researchers hoping to begin new research in this area. Hameed et al. go on to provide high level detail in addition to enumerating constructs pertaining to TRA, TAM, and TPB. Of the framework's antecedents, organizational readiness and culture are identified as being critical. Additionally, prevailing environmental properties, such as competitive pressure, are cited as important factors. 
Hameed et al.'s research is important because it contributes to identifying the models explaining specific aspects of IT adoption, seeing IT as a uniquely digital phenomenon, for instance. While this work does serve to fill gaps in the current research and combines multiple models to present a more useful model, their model would have been best served through additional empirical field study of the combined model to test its validity. Because additional field research was not conducted, there is no empirical evidence which validates the findings of the researchers.

Hameed et al. built upon previous research by Karahanna et al. (1999), which uncovered that while the IT pre-adoption behavior was well covered at that time, there was still a weakness pertaining to the temporal dimension, i.e. the initial adoption and post-adoption behavior of the technology (Karahanna et al., 1999). Taken in its entirety, thus far, the lack of research into how end-users form their initial attitudes about technology (Karahanna et al., 1999) continued through at least 2012 when Hameed et al. completed their research.

Most recently, Kupfer et al. (2016) revisited Karahanna et al. and found it is still unclear how various factors attributed to adoption behavior differ between early adopters and those who wait to adopt the same technology at a more mature level (Kupfer et al., 2016). In other words, research across 17 years had yet to offer a cohesive model for predicting and measuring the pre- and post-technology adoption behaviors within firms.

Hence, gaps in this area of research continue to be identified. Furthermore, these gaps are important to the drivers in the current dissertation pap because theory on digital transformation technology adoption adds another dynamic to traditional technology 
adoption behavior (Jin and Li, 2012) and further occludes strategic technology adoption drivers.

Conceptual Model: Impact of Alignment of the Data Network on DX Outcomes Internal drivers, such as need for more product or service capabilities, or external drivers, such as competitive market forces, cause organizations to formulate DX strategies. Irrespective of the reasons driving DX, the resultant strategy and expected outcome of the transformation can be aligned to one of the four themes of digital strategy proposed by Bharadwaj et al. (2013). Therefore, these themes can be categorized as: scope, scale, speed, and sources. Scope defines the systems to be transformed (Bharadwaj et al., 2013). Scale defines the breadth and depth of organizational functions which the transformation will affect. Speed defines how quickly the transformation will be implemented and sources are the origin of the resultant digital value creation (Bharadwaj et al., 2013).

For the purposes of this research, the focus is on large organizations undergoing, or having undergone digital transformation with high levels of scope and scale. A simple example of high levels of scope and scale would be the transformation from dependence on tellers to facilitate financial transactions to self-service software applications running on PCs and smartphones; these allow almost all financial transactions to be conducted remotely, without the need to visit physical bank branches.

Ultimately, the scope and scale of digital projects influence the level of expected digital pervasiveness within the organization. However, the traditional properties of strategic adoption of technology within organizations is constrained to the status quo and often does not compensate for the functional IT relationships of the past (Bharadwaj et 
al., 2013), that is, needing now to support new business-to-IT relationships embedded in DX strategies. Although functional components which affect technical project outcomes are numerous, this research has selected two dimensions from the many functional organization properties to simplify the logic: company size and resource capabilities.

As the balance of responsibility for implementing and managing pervasive organizational change now requires a shift from the traditional responsibilities of the CEO and other functional executives to technical executive leadership, which has historically resided in the CIO (Thomas et al., 2016), confusion around the optimal mix of resources needed for pervasive digital adoption increases (Hess et al., 2016). Therefore, this research hypothesizes that considering only traditional antecedents to DX outcomes, that is, seeing the data network aligned at the historically functional level, leads to low digital adoption levels or complete project failure.

Although data network technology had evolved as a critical factor in meeting functional organization process requirements long before the term digital transformation, these capabilities have in the past gone largely unrealized and unexploited (Andal-Ancion et al., 2003). Enterprises generally understand, and the literature makes this clear, that DX will require new information technology capabilities and resources. However, because enterprises have limited resources to devote to technology projects, irrespective of their tactical or strategic alignment, the more pragmatic focus should be on increasing higher-layer IT capabilities, e.g., applications (Andal-Ancion et al., 2003). As such, the data network continues to be largely excluded from architectural consideration during DX projects and is still often updated only during predefined refresh cycles. These 
predefined refresh cycles are often driven by the CFO rather than the CEO or CIO (Cusimano, 2013).

Given this positioning, the dissertation postulates that there are direct linkages between functional and strategic data network alignment and the success of DX outcomes. This is illustrated in the conceptual model "DX, Data Network Alignment, and DX Outcomes" depicted as Figure 1.

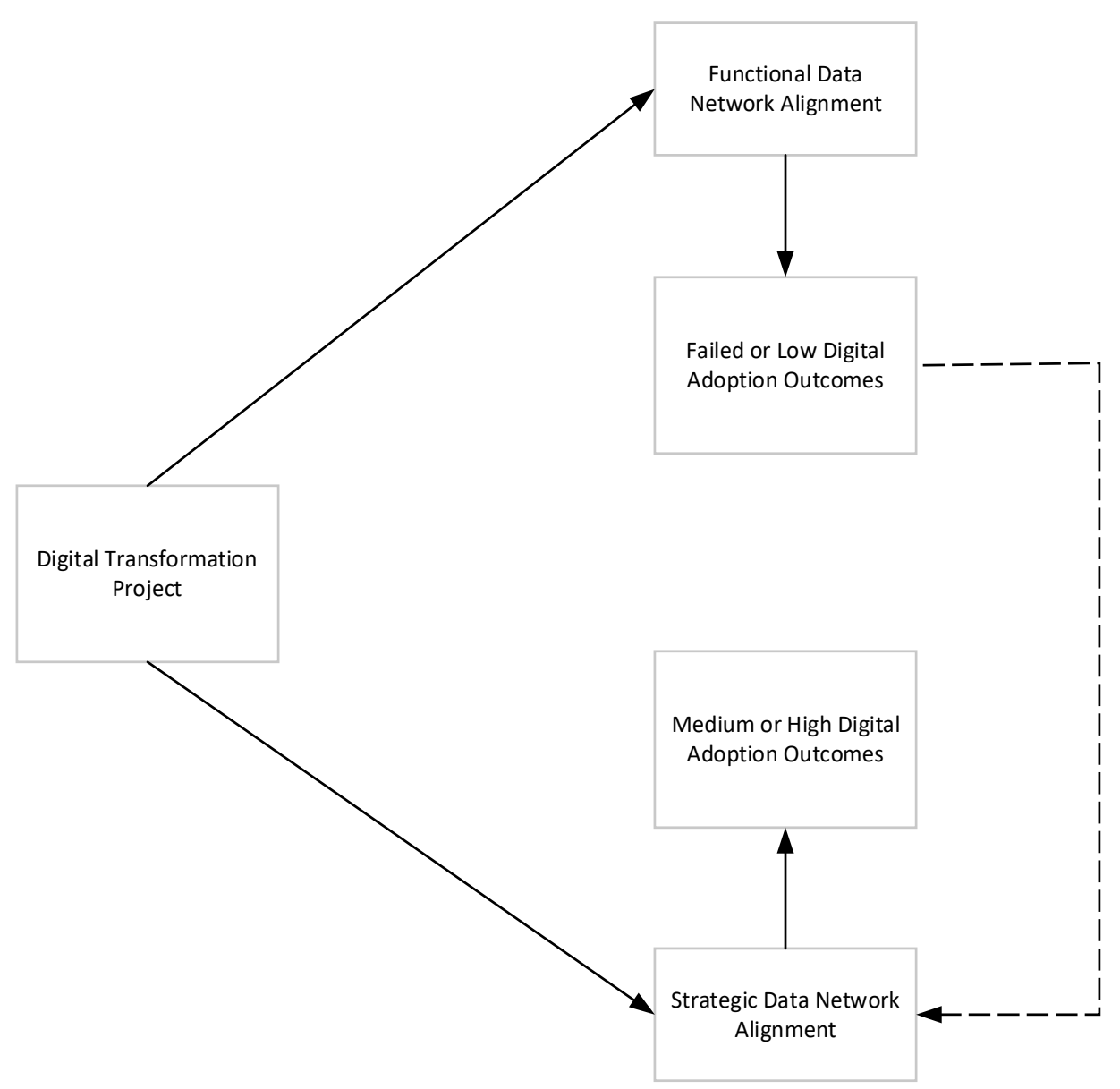

Figure 1. Conceptual Model of DX, Data Network Alignment, and DX Outcomes In this model, the strategic alignment of data network systems, and procurement of the data network specifically to support DX initiatives, have a direct impact on medium or high outcomes of digital adoption. Furthermore, the research proposes that there is a cybernetic relationship between completed or late stage DX projects which 
originally yielded low or failed results and a retroactive strategic alignment of the data network. This cybernetic effect can possibly increase digital adoption outcomes to medium or high levels. For the purposes of this research, levels of digital adoption are measured by the ability to meet a given ratio of digital transformation objectives defined in the original project charter(s) in addition to the ratio of digital adoption expected in the original project.

\section{Summary of Literature}

The purpose of the literature review was to understand the current state of research as it relates to strategic technology adoption within firms, especially as it pertains to the strategic alignment posture of the adopted technology. This research will observe DX technology adoption, the possible shift of technology which historically played a tactical role to contemporaneously playing a strategic role, and finally, attempt to measure an outcome of that process.

To this end, we began reviewing the literature with research by looking at investments of IT procurement by Sriram and Stump (2003). In that research, they point out a productivity paradox wherein merely investing money into IT does not return a consistent increase in productivity, e.g., simply throw money at the problem will not solve it. Instead Sriram and Stump (2003) observe that there is a necessary organizational interaction at a social level, to align the potential IT investment to specific needs.

Building on research into ROI for IT investment, Davern and Kauffman's (2000) argue that IT leadership has been challenged by the action of properly aligning technology to achieve optimal value from the investment. A critical issue was the inability of IT leaders to manage the transformation of many technologies from tactical to 
strategic roles (Davern and Kauffman, 2000). Wu et al (2015) found that strategic alignment of IT investments is key to achieving optimal organizational performance.

As functional-to-strategic technology transformation became a central theme of the literature, Drnevich and Croson (2013) also found that firms are struggling with properly aligning IT investments with business requirements to achieve the required outcomes. They also reinforced the human element of the decision making and alignment process Sriram and Stump uncovered in their 2003 research. Similarly, Weill et al. (2002) found that the highest performance firms have a highly mature communications process which leads to optimal IT investments and subsequent high-performance outcomes from these investments. However, Tallon (2007) uncovered that strategic alignment is not a homogeneous concept and often varies widely between firms. As a result, similar technologies adopted by similar firms often yield widely dissimilar results. Ullah and Lai (2013) also built upon Tallon (2007) by stratifying the components of strategic alignment to a deeper fit. Fit is inclusive of the properties of business strategy, IT strategy, business infrastructure, and IT infrastructure.

The dissertation next expanded on the challenges with IT strategic alignment and explored the specific connected technology properties brought on by DX and the fourth industrial revolution. As such Miller (2015) and Xu et al (2018) found that new organizational challenges were introduced by DX, to which Demirkan et al. (2017) explored how achieving IT alignment with DX requirements necessitates a prolific identification of objectives of DX at all firm levels. 
Finally, we explored the state-of-the-art of models that specifically sought to explain technology adoption. In doing so we found that there are no single adoption models which can uniformly explain how IT is adopted into the firm (Qrunfleh, 2010).

Taken in its entirety, we next found that the literature revealed many dynamics, antecedents, and variables that govern optimal strategic IT alignment for achieving optimal outcomes and ROI within firms. However, despite voluminous literature on the phenomena of technology adoption, there are still significant gaps, especially in relation to process-level dynamic relationships and their direct effects on technology adoption outcomes.

Because technology is constantly evolving, the requisite adoption behavior must evolve to consider technology trends at a given point in time. Therefore, metrics such as TAM's perceived usefulness are moving targets. It was clear that there is still much work to be done as the rapidly innovative landscape of both disruptive and incremental technologies required to support DX pose new challenges to the body of research these areas. As such we found that the research in this paper offers value to the existing body of research as we study a previously unexplored variable in IT-to-business alignment, but also how its alignment affects DX outcomes. 


\section{CHAPTER 3}

\section{METHODOLOGY}

As a reminder, we define digital transformation as the strategic restructuring of organizational processes to be able to deliver products and services more seamlessly, transparently, efficiently, and effectively to stakeholders through the use of digital technologies. By its very nature DX causes an organizational change to varying degrees, however DX need not be deemed "disruptive" to be digitally transformative (Furr and Shipilov, 2019). For the purposes of this dissertation DX projects must (1) present a shift from an existing process or technical system, (2) have a direct impact on the capabilities of end-user within the organization to complete required tasks, and (3) have a dependency on the data network for operation. Therefore, these key properties enable us to study the interdependencies which are critical to adoption, and how they factor into the subsequent DX outcomes.

It should also be noted, that since IT has been defined in the literature as a traditionally functional mechanism, the mere adoption of technology without a distinct organizational process transformation rationale would not qualify as DX. As it were, Tabrizi et al (2019) stress that DX is not about technology, rather DX enables new processes which drives more efficient information flows to and from people inside, and outside, of the organization.

With this in mind, a qualitative research approach was chosen for this dissertation because prior literature and theory were not sufficiently informative about the lack of strategic attention to the data network and the possible consequences of that lacuna as it 
relates to DX. As noted above, the data network is unusual in that it has always been interpreted as mere hardware and/or infrastructure and, therefore, not to be thought to be a strategic investment.

Amongst the many choices of qualitative methods, the case study is one of the most frequently seen in business school studies. This is because it offers the opportunity to gather rich data and to utilize this rich data to triangulate on the phenomenon of interest (Campbell, 1975). Case studies, however, have weaknesses related to external validity (Stone, 1978). Still, their ability to compare across settings where results can conceivably differ in predictable ways compensates substantially for this disadvantage (Yin 1994). In this situation, the strengths of the case study and the multiple case study outweigh their conceivable weaknesses.

Whereas these factors do not render the case study strong on internal validity, it does mitigate the criticism that it cannot examine cause and effect relationships. This makes the interpretations of qualitative studies "loosely causal" (Markus and Robey 1988, p. 579) and thus of great importance to managers, employees, and consultants who are seeking to attribute causality in some form or another to why events occur in enterprises.

\section{Rationale for Research Approach}

Yin (2014) states that case studies can be invaluable when attempting to study how and why questions, particularly in certain situations: when there is little control over behavioral events and when the focus of research is a contemporary subject (Yin, 2014), for example. Additionally, when researching phenomena which is not yet clearly understood, a pilot case study can be a powerful tool especially when there are complex 
relational interactions (Christie et al., 2000; Eisenhardt, 1989). While there is a growing DX body of knowledge, the literature still indicates that relationships and causation are not well understood on an organizational level (Yu, 2011). And no study to date attempts to include traditionally functional technologies, such as the data network, and its relationship to DX adoption.

To this end, this research began with a pilot case study which was used to test the appropriateness of the research concepts (Sampson, 2004). Sampson indicates that while pilot studies are underused, they are valuable for refining research methods and tools in qualitative research. Following the pilot case study in this dissertation, a multiple case study approach afforded the opportunity to deliver much more robust research and more compelling data versus a single case study methodology (Yin, 2014).

Additionally, because a formal study on the effect of the data network on digital transformation has not been attempted, it was deemed that a single observation of the phenomenon would not be a compelling examination of the relationship between the data network and digital transformation adoption levels. As it were, the results from a single case study could be deemed anecdotal, whereas multiple observations are much more compelling (Yin, 2014). This is especially the case where future qualitative study could be used to not only triangulate on the same relationships, or the lack thereof, but also to drive quantitative study to infer causation more definitively.

In his seminal book on qualitative methods, Myers (2013) argues that it is possible (and desirable at times) to use quantitative data to inform the case study. He refers to this as a "positivist" qualitative approach in his article on the topic (see Figure 2 below). 


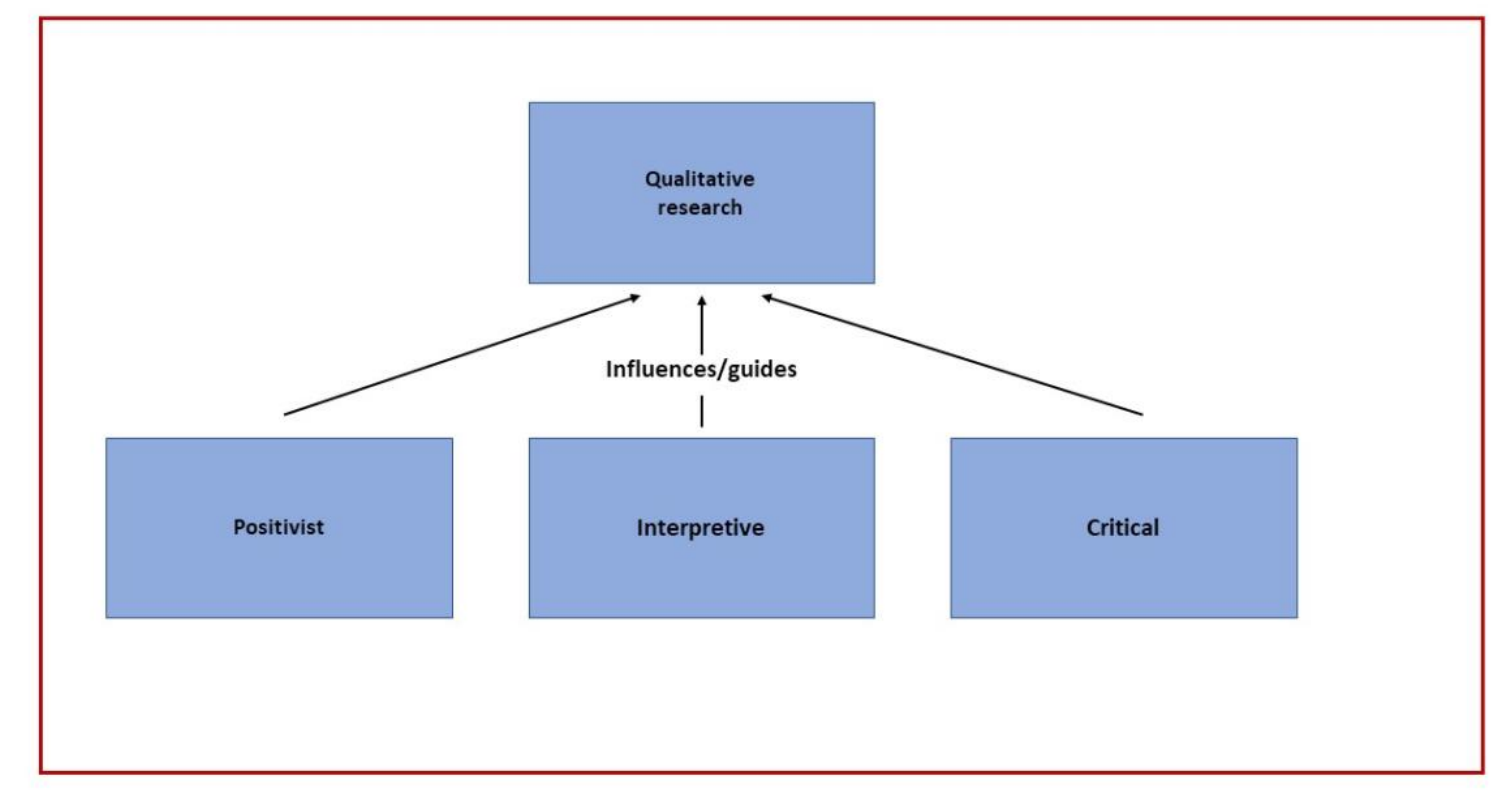

Figure 2. Positivist Qualitative Methods (Adopted from Myers, 2002). Copyright (C) 1997, Regents of the University of Minnesota. Used with permission.

Myers further says:

Positivists generally assume that reality is objectively given and can be described by measurable properties which are independent of the observer (researcher) and his or her instruments. Positivist studies generally attempt to test theory, in an attempt to increase the predictive understanding of phenomena. In line with this Orlikowski and Baroudi (1991, p. 5) classified IS research as positivist if there was evidence of formal propositions, quantifiable measures of variables, hypodissertation testing, and the drawing of inferences about a phenomenon from the sample to a stated population....Examples of a positivist approach to qualitative research include Yin's (2002) and Benbasat et al's (1987) work on case study research.

In the case of this dissertation, we found that the ability to formalize the DX

outcome variable would be invaluable for providing deeper context to the phenomena being observed. However, this approach is challenging because of the total lack of quantitative tools for calculating and measuring DX outcomes being discovered in this research 
The most relevant model found was detailed in Inel's 2019 research where he used data envelope analysis (DEA), a model which based on research by Farrel (1957), to measure DX outcomes across various countries. This model utilizes digital infrastructures, investment and access to finance, supply and demand for digital skills, eleadership, and entrepreneurial culture as inputs, with DX as an output. However, research outputs such as DEA assess DX to measure macroscopic performance, whereas, this dissertation requires a model which can be used to measure the narrow output of the DX project irrespective of the macroscopic effect on the organization at large. This gap was subsequently addressed through the formulation of the Digital Outcome Raw Score (DORS) which was to become the keystone of this dissertation.

DORS was formulated in order to measure the possibility of "loosely causal" relationships (Markus and Robey 1988, p. 579) that might exist between how organizations strategize about data networks and whether this leads to successfulunsuccessful DX implementations. Having a dependent variable metric gives us a way to attribute possible causality, even if it serves mostly as a prompt for future research. DORS is covered in detail in the upcoming Units of Analysis Section of this paper.

How the Selected Method Addresses the Research Questions As a reminder, the four RQs for this dissertation are:

(1) In what respect does DX necessitate that firms transform their procurement drivers of the data network from tactically aligned to strategically aligned?

(2) How are IT investments in the data network related to digital transformation project outcomes?

(3) How has DX caused data network adoption and consumption decisions to evolve? 
(4) How are DX outcomes measured and are current models sufficient for describing DX project outcomes with the required levels of precision?

The qualitative methodology in this dissertation was selected specifically to address the lack of addressable research on the influence of the data network on DX outcomes. Additionally, the direct impact of IT investments specifically on DX projects, and the transformative role of the network specifically as it relates to DX have been studied in their constituent parts but have not been researched for the cross-functional effect on DX project outcomes. Finally, there simply was no method for measuring the level of DX outcome with any precision.

As a result, the direct observation was necessary to substantively understand and document the intrinsic dynamics of the decision-making process, resource fit, and associated DX project outcome. However, there was a distinct opportunity to not only offer a proposition on the phenomena but develop a model in an attempt to explain the findings (Danis and Parkhe, 2002. Myers, 2013) which interpretative analysis alone was insufficient to provide, hence the formulation of the DORS model. Thus, the benefits of the resultant DORS model are twofold. First it offers the precision to support the qualitative analysis of this research to support lose causality. It also offers a starting point for future quantitative research to validate that causality.

Metrics and Evaluations

For the purposes of this research, strategic application of technology is assessed through Chandler's requirement (1962) that it be aligned to the long-term goals of an enterprise and the adoption of courses of action and allocation of resources necessary for carrying out these goals. Strategic alignment of the data network should support a short, 
medium, or long-term organizational mission and integrate with complementary technologies to form a combined technology platform that will undergird the digital transformation.

Conversely, functional alignment of the data network is predicated on simple, operationally focused, technical requirements such as basic connectivity, resiliency, and other data network properties, the requirements of which generally originate within the IT department. Functionally aligned deployments represent status quo data network procurements whose goals are to remediate equipment at end-of-life or end-of-support by the vendor or has simply become inoperable, causing an outage or degradation of service. These are typically lifecycle or remediation related procurements which align to technology refreshes which are cyclically conducted during a predefined refresh cycle when the vendor has declared that the equipment will no longer be supported or simply as an immediate break-fix response.

Finally, the outcomes derived from data network alignment should include the capabilities of the enterprise to successfully deploy the technology in direct fulfillment of the initial procurement requirement justification. For instance, the organization may not have the appropriate resources to adequately deploy the data network to support the digital project despite meeting the strategic procurement metrics. Conversely, the original procurement could have been set forth for reasons unassociated with digital transformation, but still well support the digital project. In such a case, an alternative relationship must be explored.

Therefore, the outcomes of data network procurement or deployment may not be linear as it pertains to the initial procurement rationale and the outcomes achieved by the 
deployment and adoption of the technology may differ from the original intent. It is possible that a functional technology application could ultimately fill a strategic requirement, and conversely, it is possible that a strategic purchase never rises above fulfilling a functional role.

\section{Units of Analysis}

The unit of analysis in this dissertation is the adoption of data network. The outcomes that are expected are, therefore, various levels of digital transformation adoption; these levels have broad and diverse organizational connotations as they pertain to scope and scale. However, there is currently no universally accepted evaluative tool for assigning levels of success or failure of digital adoption within an organization because not all organizations will achieve similar levels of digital adoption despite having similar capabilities and resources to apply to the digital project (Zhu et al., 2006). Zhu et al. go on to indicate that usage continues to be a "missing link" (p. 11) in digital transformation adoption research. As a reminder, Yu et al (2011) spoke at length to the lack of measurement of organizational adoption of technology. To this end it was deemed that a scale by which DX outcomes could be measured is an essential component of this research. Hence the scale developed to meet these requirements is the Digital Output Raw Score (DORS).

Therefore, for the purposes of the pilot, and subsequent multiple case studies,

DORS is used as the measurement of dependent variable attainment. DORS expresses DX project deliverable attainment (outputs) thus:

$$
\begin{aligned}
& 1.0-.8=\text { High } \\
& .79-.60=\text { Medium-High } \\
& .59-.40=\text { Medium }
\end{aligned}
$$


$.39-.20=$ Medium-Low

$.19-.01=$ Low

$0=$ Null

DORS covering the following attainment states:

- Low Adoption: The digital transformation project failed to be completed or post project adoption of the associated blended digital transformation deliverables of the project was $0 \%$ to $19 \%$.

- Medium-Low Digital Adoption: The digital transformation yielded low-tomedium/low levels of post project adoption of the associated digital transformation deliverables of above $20 \%$ and below $39 \%$.

- Medium to Medium-High Digital Adoption: The digital transformation yielded medium-to-medium/high post project adoption of the associated digital transformation deliverables of above $40 \%$ and below $79 \%$.

- High Digital Adoption: The digital transformation yielded high levels of post project adoption of the associated digital transformation deliverables greater than $80 \%$.

- Null / Failure: We felt that the DORS representative of DX project failure should be left to the sole judgement of the organization. Because both success and failure are subjective, and dependent on a seemingly infinite number of factors, it is out of the scope of this dissertation to declare a representative DORS which signifies failure of the DX project. Rather we present a DORS which is indicative of the DX project results and allow each organization to make its own decision on the data.

In addition to constructing a scale by which DX project outcomes could be measured, a formula for calculating the outputs was necessary. The formula used to calculate DORS is illustrated in Figure 3. 


$$
\begin{aligned}
& \left(\frac{a x_{x}}{\partial x_{x}}\right)=a_{x} \\
& \left(\frac{o x}{d x}\right)=0,0 x,=0 x
\end{aligned}
$$

Figure 3. Digital Outcome Raw Score (DORS)

The variable $\mathrm{DX}_{\mathrm{d}}$ represents the total project deliverables of the original DX project charter. Variable $\mathrm{DX}_{\mathrm{a}}$ represents the project subset of deliverables realized from the original project charter. Similarly, variable $\mathrm{DX}_{\mathrm{p}}$ represents the total target population of the DX project, and the variable $\mathrm{DX}_{\mathrm{u}}$ represents the estimated users which have fully adopted the DX technology after project completion. The variable DXo represents the sum of the other variables in the formula, e.g., the DX project outcome. DX $\mathrm{X}_{\mathrm{o}}$ is the representative integer of DORS achieved and is ultimately used to measure the outcome of a tactical/functionally aligned vs a strategically aligned data network. Therefore, the formula enables a precise score to be assigned to DX outcomes from low to high. It is undoubtedly a key contribution of this research if it is validated during the course of the research.

Because the definition and measurement of strategic alignment of the data network must be classified for the purposes of this research, it is predicated upon the procurement decision rationale of the current network procurement. The drivers for the procurement, would usually originate outside of the IT department and address a tangible set of business or technology requirements, e.g., business mission driven DX initiatives to 
which a technology solution is applied. DORS is therefore a straight-forward raw score without the direct effect of other possible contributors to the overall DX outcome.

\section{In-scope Technologies}

The data network technologies included in the pilot and multiple case studies meets the commonly accepted definition of the data network: a digital telecommunications technology which enables designated nodes to communicate and share resources through the transfer of data (Bourgeois and Bourgeois, 2014). Therefore, the data network systems analyzed in the pilot and case study studies are inclusive of:

- Local Area Network (LAN): Inclusive of single building, or interconnected local campus wired and wireless network switching equipment

- Wide Area Network (WAN): Inclusive of network routers which transfer data between nodes or locations usually located more $.80 \mathrm{~km}$ apart, but not considered to be inclusive of a single local campus

- Data Center Network (DCN): Network equipment specific to a local datacenter which provides routing and switching capabilities directly between servers and storage systems, and end-users

- Network Security: Dedicated firewall appliances (including virtualized instances)

- Storage Area Networks (SAN): Network switching and routing equipment which provides dedicated high-speed connectivity specifically for flash, and traditional disk storage arrays

- Servers and server infrastructures are specifically excluded

- Storage arrays are specifically excluded

\section{Pilot and Multiple Case Studies Participant Sources}

The scope of the case studies necessitated target organizations to have a large scale of operations, and complex technology requirements. This was required because the 
impact of respective cross-functional relationships would be consequential at the macroscopic organizational level.

For the purposes of the study, "large scale" was defined as operations for which DX would affect 1,000 individuals at a single site or for which DX would affect regional, national, and global operations with at least 2,000 individuals. Companies where identified as having complex technical requirements if the data network architecture was designed with a Wide Area Network (WAN) to account for regional or global distribution of sites, and/or for high capacity branch offices and corporate campuses with at least 1,000 individuals in a single location. Furthermore, complex technical requirements were inclusive of multiple departmental software application systems running in either onpremises data centers or in cloud-based network infrastructures.

Target firm sizes were restricted to those that met the accepted definition of being a large firm, e.g., having more than 500 employees. As of the 2012 Census, there are approximately 16,000 such firms in the United States that meet the criteria, thus offering a wide potential research population that could be generalized to in later work.

Finally, the target firms must be undergoing or have recently undergone multiple projects that meet the definition of being digitally transformative within the last 36 months. Preferably, these firms will also be in the process of performing a data network technology refresh or will have performed such a refresh within the last 24 months; however, not having performed a data network technology refresh within this timeframe was not a disqualifier. 


\section{Confidentiality of Data}

Because the invasive nature of case study research requires direct observation and in-depth gathering of data for each case study participants, much of which is considered sensitive, all data from all study participants was gathered under a guarantee of anonymity. An IRB approval from Temple University was sought and acquired for the purpose of ensuring that the risk of harm to participants was low or a good trade-off with the benefits derived from the study. In this case, anonymity lowered risk to subjects.

This should not be an issue with regard to the quality of the findings. It is standard practice in case study research to anonymize data to protect the privacy of the participants while simultaneously enabling the availability of the data for research (Thomson, Bzdel, et al., 2005). Moreover, the protection of individual privacy did not hinder the attributions of effect to possible antecedents by the researcher.

The subjects for the pilot and multiple case studies were chosen between August and December 2017. The pilot case study began in January 2018 and was concluded in March 2018. The multiple case studies were conducted from September 2018 through January 2020. The multiple case study was designed to support a 2 x 2 observation, where two companies that achieved high levels of DX outcomes and two companies that achieved low levels of DX outcomes where observed. Table 4 provides an overview of the anonymized study participants from all studies. 


\begin{tabular}{|l|l|l|l|l|l|}
\hline Table 4 \\
Pilot and case study participants \\
\hline $\begin{array}{l}\text { Participant } \\
\text { Code }\end{array}$ & $\begin{array}{l}\text { Industry } \\
\text { Vertical }\end{array}$ & $\begin{array}{l}\text { Number of } \\
\text { Employees }\end{array}$ & $\begin{array}{l}\text { Network } \\
\text { Upgrade? }\end{array}$ & $\begin{array}{l}\text { Digital } \\
\text { Transformation? }\end{array}$ & $\begin{array}{l}\text { DX- } \\
\text { Adoption } \\
\text { Level } \\
\text { achieved }\end{array}$ \\
\hline PCS-1 & $\begin{array}{l}\text { Higher } \\
\text { Education }\end{array}$ & 2,500 & $\begin{array}{l}\text { Yes - In } \\
\text { progress }\end{array}$ & $\begin{array}{l}\text { Yes - In } \\
\text { progress }\end{array}$ & $\begin{array}{l}\text { Medium- } \\
\text { Low }\end{array}$ \\
\hline MCS-1 & Retail & 30,000 & Yes & $\begin{array}{l}\text { Yes - In } \\
\text { progress }\end{array}$ & High \\
\hline MCS-2 & Automotive & 650,000 & $\begin{array}{l}\text { Yes -In } \\
\text { Progress }\end{array}$ & $\begin{array}{l}\text { Yes - } \\
\text { Postponed }\end{array}$ & Low \\
\hline MCS-3 & Manufacturing & 250,000 & Yes & Yes & Medium \\
\hline MCS-4 & Airline & 4,000 & Yes & Yes & $\begin{array}{l}\text { Medium- } \\
\text { High }\end{array}$ \\
\hline
\end{tabular}

Legend: PCS- $n=$ Pilot Case Study $n$. MCS- $n=$ Multiple Case Study $-n$

Techniques for Data Collection and Participant Observation

Multiple techniques were used to afford a sufficiently robust and dynamic collection of data as shown in Table 5 .

\begin{tabular}{|l|l|}
\hline \multicolumn{2}{|l|}{$\begin{array}{l}\text { Table 5 } \\
\text { Research technique rationale }\end{array}$} \\
\hline Direct Observation & $\begin{array}{l}\text { Enables monitoring of the dynamic interactions of } \\
\text { technical, business, executive, and digital project stake } \\
\text { holder teams which would not be captured solely } \\
\text { through interviews and surveys (Merriam, 2009) }\end{array}$ \\
\hline Semi-structured Interviews & $\begin{array}{l}\text { Provides interviewee more latitude to build upon } \\
\text { thoughts and concepts during interviews and affords } \\
\text { more depth vis a vis structured interview. (Myers, } \\
\text { 2013) }\end{array}$ \\
\hline Embedded Observation & $\begin{array}{l}\text { Allows for a multiplicity of methods to be applied and } \\
\text { is especially useful in complex cases (Scholtz, 2002) }\end{array}$ \\
\hline Multiple Case Study & $\begin{array}{l}\text { Offers more compelling and robust observation of the } \\
\text { phenomena vs a single case study. (Yin, 2018) }\end{array}$ \\
\hline
\end{tabular}


Data was gathered through direct observation during an embedded period of at least one week and semi-structured interviews. Direct observation of operational and executive teams was conducted to enable a contextual understanding of the current and proposed network environments, DX project deliverables, resource capabilities, and the team dynamic of all direct and indirect project stakeholders. Because team capabilities and dynamics are integral to project outcomes (Guang-dong, 2013), it was important to understand the core relational, organizational, and technical capabilities of the team and their effect as an influencer on project success (Young-Hyman, 2017). These relationship dynamics were observed over the embedded period.

Poor technical skills and governance capabilities are criteria for disqualification of a case study candidate because the strong negative moderation on the overall project outcomes could affect the results of the study. In such cases it is possible that even successful technology alignment alone is not be enough to overcome the negative capabilities influence on the overall project outcome.

Thus, to sufficiently capture the depth and complex dynamics of the technology, business, and human interactions, a multiple case study approach provides the desired observational variability of the phenomena to understand the differences and the similarities between the cases (Stake, 1995; Baxter and Jack, 2008). Because the scope of the research requires an in-depth observation of how and why the phenomena occurs, a deep observation of the behavioral events is key. Additionally, because the research is of a contemporary phenomenon, the case study presents an optimal fit (Yin, 2014).

Yin (2014) offered the following case study tactics (reflected in Table 6) that form the basis for structuring both subsequent multiple case study designs. 


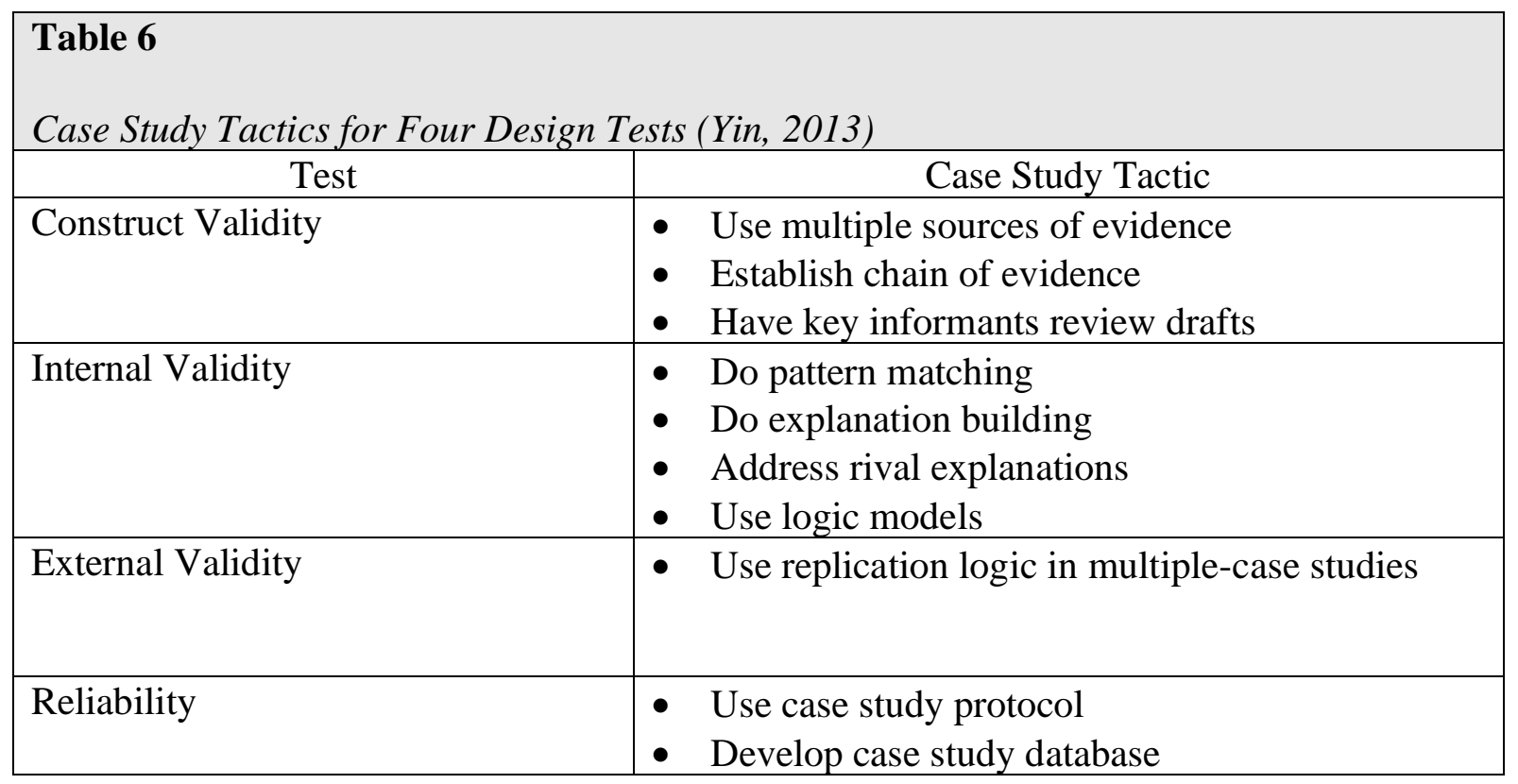

The research subjects include two organizations that have achieved high digital transformation outcomes and two organizations that have achieved low digital transformation outcomes, or experienced outright DX project failure. This kind of contrast in outcomes is highly recommended by Yin (2014).

Next, semi-structured interviews were used during both the pilot and multiple case study to collect data. Interviewees were chosen from a cross-section of executive, business, technical, project management, and procurement resources as follows:

- Chief Information Officer (CIO) or technical director level executives

- Data Network Manager / Supervisor. (Note: In more than one occurrence data network responsibility was split between local, wide area, and data center networking leaders.)

- Data Network Engineers and Architects

- Procurement manager/lead responsible for data network procurement.

- Chief Operating Officer or other business director level executives

- Program and project managers

- Technical contractors working as data network subject matter experts. 
Individual semi-structured interviews were used because they are designed to be less formal and elicit an open-ended discussion rather than a single answer. The format also lends itself to rich conversation that may branch off to ancillary, but relevant areas that may not be readily apparent. Additionally, the research covers relatively nascent areas of DX research; therefore, there was an opportunity to uncover and associate patterns relevant to the research but less likely to be touched upon during the more rigid structured interviews. Participants were encouraged to elaborate on any given question as the subject felt necessary. Furthermore, because many comments were related to peer, management, and overall business performance in relation to technical and business capabilities, no group interviews were conducted, and confidentiality of all interviews was thereby guaranteed.

\section{Instrumentation of Interview Schedules/Scripts}

The following are a subset of questions taken from the interview guide, included in Appendix A, and used to establish the subject's understanding of various business, project, and technical areas.

1. In your own words, what is digital transformation?

2. Tell me about the business drivers for any projects which your company deems to be part of digital transformation, currently in progress or that you have completed over the last 18 months.

3. Detail the project deliverables of those projects which your organization designated as digitally transformative?

4. Why did you last upgrade the network?

5. When company employees complain about the data network - what are their primary complaints and how do they state that the reasons for their complaints affect them and their work?

6. What are your thoughts of the new application? 
7. Do you feel that the new application has made you more, or less efficient? Why?

When developing the interview questions, separation of questions by specific expertise and job function was deliberately avoided. The goal was not only to understand the cross-functional interactivity of the DX projects, but to gauge at what level all project stakeholders were familiar with the cross-functional business and technical components necessary for a successful project outcome. Therefore, the research measured the level of technical understanding and how the data network impacted DX.

Furthermore, the research tested to what extent interviewees were architecting the data network specifically to support digital transformation. But interviewees were not led by the questions to say that the data network was either strategic or functional; their perceptions emerged naturally from their unprompted responses. In brief, there was no social desirability attached to their intuitive responses.

Similarly, the research was designed to understand at what levels executive, procurement, and project resources could identify and detail deficiencies with the current network. Capabilities in these areas also provides indicators to the levels of interactions between the various teams and stakeholders. These interactions are vital to integrate technical and business requirements and direction thereby jointly defining the unified strategic direction for DX as it relates to the data network and vice-versa.

The interviews were recorded with an Apple MacBook Pro computer and stored/secured on a removable USB drive. The unedited recordings were subsequently transcribed verbatim by a third-party transcription service. Because direct observation was used and direct interaction with the respective teams who were critical stakeholders in the DX project and data network procurement process was integral to the research, it 
was not necessary to gather survey data because it would be redundant to the direct observations, interviews, and notes.

The resultant transcoded interviews were compiled and coded using techniques outlined in Saldaña's The Coding Manual for Qualitative Researchers (2016) to synthesize, categorize, and code the data. NVivo was then used to support coding and modeling the data analysis against the conceptual research model. No codes were formulated prior to the actual review of the data.

Analysis of the data began with open coding and subsequently codes were attached to text via NVivo. An example of a code developed after data collection was completed from the pilot case study is: NDTNT3SDN_Bandwidth, which indicates that the evidence fits the code "bandwidth" which was collected during an interview with network team member 3 (NT3) who worked on a project that was designated by the organization as non-digitally transformative (NDT) and referred to as the Student Dorm Network (SDN) upgrade Project.

The codes were subsequently compiled into categories divided into digitally transformative and non-digitally transformative types, with properties that affect each project type in relation to the data network.

\section{Reflexivity and Trustworthiness}

Yin (2018) described reflexivity as a barely perceptible influence of the interviewer on the interviewee. Conversely reflexivity can also influence questions being asked of the interviewee. The resultant effect of reflexivity is inaccurate data which will have a negative effect on the data being observed, compiled, and subsequently reported. 
Likewise, if the data inputs cannot be trusted, then the outputs, observations, and indeed the research cannot be deemed accurate

Reflexivity and trustworthiness are concerns during direct observation of behavior because as subjects are being observed, they are cognizant of being observed, thereby eliciting contrary behavior to not being under observation (Mauthner and Doucet, 2003). Reflexivity is also a concern when collecting data from interviews because the subjects are agents of prominent companies. As such, it is possible that their answers are given in such a way to mask weaknesses in corporate operations, protect intellectual property, or to spin an outcome with a more positive lookout. Therefore, any behavior that seemed anomalous or contrary to the outcomes were identified and noted. A "cradle to grave" method of observation and data collection was adopted to study not only the post-project level of digital adoption but the evolution of the data network throughout the course of the digital project. Interview data were collected on the level of understanding of the overall corporate tactical and strategic goals, as well as the depth of understanding of strategic data network related technologies. The goal is to build a 360-degree understanding of the behavior such as:
A) What is known?
B) What was done?

The Relevant Descriptive Characteristics of:
A) What was the result?
B) What was done before?
C) What was the prior result? 


\section{Pilot Case Study Setting}

The pilot case study was conducted at a large and prominent research university, designated PCS-1, located in the midwestern United States. The university was founded in the mid-twentieth century and has a total academic and operational staff population of over 2,500 with a student population of over 28,000 . The university campus has multiple large campuses consisting of over 100 buildings spread out over almost 250 acres. The university is experiencing continued growth of both the student and employee population which is driving major indicatives to digitize learning systems and enable new collaborative capabilities for staff and students. The university currently has an extensive and complex data network infrastructure; however, the infrastructure is aging and disjointed. In addition to having many digital initiatives, the university is in the process of updating its entire data network and network security infrastructure. Consequently, the combination of organizational size, digitalization initiatives, and data network projects provides an ideal candidate to observe the dynamics of the cross-functional and strategic dependencies of the respective projects and supporting technology infrastructure.

\section{Multiple Case Studies: 1}

The first case study was conducted at a large retail chain headquartered in the Southeastern United States, and designated MCS-1. This $\$ 8$ billion USD retail company was founded in the early twentieth century and today has almost 30,000 employees, 1800 stores, and a presence in 49 states. The market in which this retailer exists is extremely competitive; therefore, it is applying technology in hopes of maximizing profits. MCS1's technology initiatives include supply chain process optimization, conversion of all business units to digital operations, and utilizing customer facing technologies, such as 
self-checkout, to enhance customer experience at each of its stores. As is to be expected, the retailer has an extensive and pervasive data network which connects not only its stores and point of sale systems, but also provides Wi-Fi based Internet access for customers. Because the company is in the process of upgrading its network and has significant digital transformation initiatives in progress, it was suitable for inclusion in the multiple case studies.

\section{Multiple Case Studies: 2}

The second case study was conducted at a global automobile manufacturer headquartered in Europe, designated MCS-2. The company has over 650,000 employees and 2018 revenues in excess of $\$ 270$ billion USD with most of its sales coming from Asian markets.

The automobile market has been undergoing significant disruption from new entrants, such as Tesla, which are technology companies first and transportation companies second. As such, automobile companies have been scrambling to digitize and further automate manufacturing. Additionally, the optimization of supply chain and internal operations are key to maximize profits. Finally, legacy automobile makers are under pressure to deliver new and innovative products to consumers.

Organizations in the manufacturing sector are quickly adopting and integrating connected sensors and Internet of Things (IoT) devices which rely on network connectivity. Indeed, the automobiles themselves are now IoT devices with self-driving, and neural networks enabling machine learning and the sharing and collection of data both between cars and with the manufacturers themselves. Automobile manufacturers like this case study participant are aggressively digitizing and the need to glean data from 
remote sources to support all areas of enterprise operations will put significant pressure on their existing data networks.

\section{Multiple Case Studies: 3}

The third case study, designated MCS-3, was conducted at a global manufacturer headquartered in the United States. MCS-3 manufactures diverse products covering consumer, commercial, and transportation sectors with 2018 revenues of more than $\$ 120$ billion USD and over a quarter of a million employees. Over the last 15 years, MCS-3 has encountered substantial disruptive headwinds that have negatively affected profitability and competitive levels against both incumbents and upstart market entrants. The company invested heavily in pervasive global digitalization to optimize operations at all levels as well as to integrate forward leaning technologies such as IoT into its manufacturing processes. This also enabled them to kick start big data enabling capabilities by harvesting real-time data from products in the field. The company expects to invest heavily in big data to be proactive in identifying patters that will improve its products, be more responsive to customers and markets, and increase profitability by streamlining manufacturing, supplier, and administrative operations.

Multiple Case Studies: 4

The final case was an airline headquartered in the United States, designated MCS4. Founded in the 1990s, the company has annual revenues in excess of \$3 billion USD. While the airline market has been highly competitive, there has historically been little disruption in the industry. Hence, MCS-4 has historically encountered little motivation to disrupt its own status quo. 
The airline market is not recognized as a growth market: Barriers to competitive entry are high, competition is high, and margins are low. As a result, to drive maximal profit from each mile flown, MCS-4 has resorted to delivering minimal services at base prices while charging passengers fees for additional incremental services to increase profits while cutting investments in technology to the bare necessities.

However, despite the traditional technology lag by the MCS-4 and its competitors, MCS-4 has identified demonstrated capabilities to drive efficiencies by DX which could deliver profit and competitive advantages.

For instance, new technologies such as electronic flight bags for pilots now replace paper charts. Onboard point of sale systems for use by the flight attendants now replace paper money transactions. Electronic bag tagging and tracking decrease the chances of lost luggage and allow customers to track their luggage in real time on their smart phones. Self-check-in kiosks reduce new number of check-in agents needed, thus reducing cost, and lowering the time customers spending waiting in line to check baggage, which increases customer satisfaction. Finally, IoT devices and sensors enable data tracking of everything from aircraft performance, wireless equipment location technology to streamline line and maintenance operations. All these technologies could conceivably have a significant positive effect on profit margins and bottom-line revenue.

MCS-4 realized that capital investments in DX technology is necessary to drive pervasive organizational optimizations across its entire operations infrastructure. The company hopes that if it can increase customer satisfaction then it can use the profits to invest in company expansion, new aircraft, and other capital investments necessary to drive and retain increased market share. 


\section{CHAPTER 4}

\section{PILOT CASE STUDY - AN INSTITUTION OF HIGHER EDUCATION LEARNS A LESSON IN DIGITAL TRANSFORMATION}

A focal university, anonymized with the designation PCS-1, was observed for the purposes of conducting a pilot study. PCS-1, with over 30,000 faculty and students on a single campus in the midwestern United States, is undergoing pervasive digital transformation as it strives to support the technical realities of the pervasive integration of technology as a key teaching and learning mechanism. Additionally, the university wished to support "bring your own device" (BYOD) requirements (i.e. privately-owned laptops, smartphones, X-box, Alexa, etc.) for its student population living on campus. As such, the university is implementing cloud based digital learning technologies for use by both the faculty and students in addition to supporting personal student devices within its infrastructure and updating its campus wide point-of-sales systems.

Current digital learning application technologies are designed to run on handheld devices such as those that run Android or Apple iOS. Additionally, these applications can run on Microsoft Window-s or MAC OS-based traditional PCs. These learning applications are increasingly being utilized by all the respective colleges within the university as an integral part of delivering the respective university curricula.

Additionally, students increasingly use the platform to take tests and instructors also use the system to grade and deliver messages and feedback to the students. The benefits that the university aspired to receive from these applications are: the standardization of delivery of the curricula, having a central repository for the extensive general university learning and specialized individual college curriculum resources, 
enabling nontraditional learning where students can access the instructor, the classroom and general university resources from off-campus from their choice of devices. The overall perceived benefits of these learning applications are such that they are becoming a normal and expected education tool throughout higher education.

In addition to deploying campus-wide learning applications the university must support over 10,000 students living on campus in the dorms. BYOD enables the organizations to support any device on which authorized users wish to work without the burden and expense of having to issue, track, and support the multitude of devices which are available to end-users. Additionally, BYOD enables end-users to work on any device with which they have the greatest comfort level, which often increases productivity and reduces learning curves necessary when adopting new technologies (Song, 2014).

As such, the university must provide pervasive wireless internet for the students to access university resources as well as the Internet. Additionally, the university enables connectivity for students' entertainment devices such as Microsoft's Xbox, Sony's PlayStation, Amazon's Alexa, and Google Home devices. The university also provides capabilities for students to connect their personal mobile phones for Wi-Fi calling because local cellular carrier network coverage is often insufficient or cannot penetrate the thick cinderblock walls of the typical dormitory or classroom building.

The university's leadership indicated that one of their top priorities are to grow student population by double digits year-over-year. Additionally, it is a top priority for the university to attract the highest quality academic instructors. Leadership stated that continued student population growth is necessary to drive income, which will in turn 
enables the university to invest in more resources and facilities to expand the scope and positioning of the university in a highly competitive higher education market.

University leadership also stated that levels of continued growth is a benchmark by which to measure the university's ability to meet and exceed its stated mission: to provide the widest access to its educational resources. In addition to educational and student related technology requirements, the university has significant operational technology requirements. The university is attempting to upgrade its point of sale systems in its campus bookstores and parking garages, Internet of Things (IoT) devices such as smart HVAC and other connected building systems, and its safety systems with video surveillance and emergency call boxes throughout the campus. There is a specific sense of urgency regarding safety capabilities because the university's campus is in an area that experiences a substantially higher level of crime than other areas of the city

As such, the DX projects underway at the university are pervasive and directly affect both academic and general operational areas inclusive of students, faculty, operations personnel, and emergency services teams. Additionally, the central thread of all DX technologies, summarized in Table 7, is their dependence on the data network for connectivity both to other internal systems, to the Internet, and to the public cloud. 


\begin{tabular}{|l|l|}
\hline \multicolumn{2}{|l|}{ Table 7 } \\
PCS-1 Digital Transformation Initiatives \\
\hline Digital transformation technology & $\begin{array}{l}\text { Network technology dependency (by } \\
\text { order of precedence) }\end{array}$ \\
\hline $\begin{array}{l}\text { Project 1: College Specific Digital } \\
\text { Learning Applications }\end{array}$ & $\begin{array}{l}\text { Local Area Network (LAN) } \\
\text { Campus Area Network (CAN) } \\
\text { Data Center Network (DCN) } \\
\text { Internet }\end{array}$ \\
\hline $\begin{array}{l}\text { Public Cloud } \\
\text { Project 2: Bring Your Own Device }\end{array}$ & $\begin{array}{l}\text { LAN } \\
\text { Internet }\end{array}$ \\
\hline Project 3: Pervasive Campus Side Wi-Fi & $\begin{array}{l}\text { LAN } \\
\text { Internet }\end{array}$ \\
\hline Project 4: Upgraded Point of Sale System & $\begin{array}{l}\text { LAN } \\
\text { Campus Area Network (CAN) }\end{array}$ \\
\hline Project 5: Video Surveillance & DCN \\
\hline Project 6: Campus wide Emergency Call & CAN \\
System & DCN \\
\hline & PAN \\
& PSTN Switched Telephone Network \\
\hline
\end{tabular}

The university has both general technology requirements that affect the entire university and specialized technology requirements that affect only specific colleges within the university. A centralized technology organization acts as on behalf of both the university and the individual colleges to maintain a pool of technology resources and technical leadership. The technology business unit is led by the university's CIO and has technology directors responsible for varying technical areas such as network, applications, security, etc., each of whom reports to the CIO and focus on a specific technology area.

The academic technology business unit has a staff of approximately 150 which is inclusive of four dedicated members of the technology project management team, ten members of data networking team, 10 members of the educational applications team, and 
other technologists that cover a host of other technical aspects of the university. The IT organization operates in a matrixed structure; therefore, all members of the technical project management and networking teams are involved directly in the current digital transformation and network refresh projects.

The university also has a governing IT committee with representatives from each of the individual colleges within the university. The goal of the IT committee is to align the operational requirements of the colleges to technology procurements and projects, which are then executed by the central technology organization.

The CIO, Director of Project Management, and Director of Networks and Telecommunications were participants in the semi-structured interviews. Five members of the networking team defined their skillsets as being specifically data networking and were included in the interviews. The remaining five members of the networking team, self-identified as security- or telecommunications-focused were therefore excluded from participating in the pilot study, despite their participation in the network refresh project.

All interviews were recorded, transcribed verbatim, and manually coded. The purpose of coding in quantitative research is to identify emergent themes resulting from the interview (Saldaña, 2016). Since the interviews were semi-structured, open-ended coding provided structure and greater capability to identify if certain key words, phrases, and patterns recur for a given question (Saldaña, 2016).

\section{Factors Related to DX and Adoption}

During open coding, several characteristics of DX and digital adoption emerged. These characteristics were specifically related to pre-project perceived functional and strategic need, project objectives and deliverable alignment, post-project deliverable 
attainment, and end-user functional adoption level. These characteristics evolved throughout the duration of the data analysis process and were compared to the existing theories outlined in the literature. Coding allowed these characteristics to be categorized and expressed as hypotheses. Subsequently, the conceptual model was based on the observations of the participants in the study, and the outcomes and adoption levels of the digital projects.

Examples of adoption initiatives that were designated as failed and/or low digital adoption were expressed as follows:

- DA0: "We continually go back to the same problem areas in the colleges and we continue to get the same complaints about the wireless network. The professors have stopped using the new learning application for their classes and we're under the gun from the Provost to fix the issue" [DTMGRLAP_WirelessStudentApp]

- DA1: "But now the card swipe application is losing contact with the validation server, so cars are getting stuck in the garage. Can you imagine this happening at five p.m. when everyone is trying to go home? So, the garage attendants regularly must just open the gates and not charge the cards, so we end up losing money." [DTPDRREV_GarageCardSwipeApp]

- DA1: "We're not trying to reinvent the wheel, so we base our project management methodology on PMBOK and our service delivery on ITIL. But I don't think we've had the network team involved in planning or in business meetings. I don't know if they've got the skillset and they're pretty busy with daily operations and multiple projects." [DTPMOREV_GarageCardSwipeApp] The data indicates that the data network is a common thread across observed DX

projects. Whereas the data network is a critical component required by the new digitally transformative applications, PCS-1's data network was identified as being tactically aligned, e.g., procured to meet basic connectivity requirements. The potential lack of strategic alignment of the data network was identified prior to the start of the DX projects and, even though the data network was refreshed as part of a specific data network in upgrade project in conjunction with the various DX projects, it never evolved past meeting only tactical business requirements. 
To explore alternative explanations for the relationship between the low digital adoption rates and the data network performance, the project management methodology was reviewed in detail because overall project failure or low adoption rates may be attributable to poor project management. However, the PMO Director held a current Project Management Professional (PMP) credential and his most senior PMO Assistant Director was recently certified in Information Technology Infrastructure Library (ITIL) Version 3 Foundational.

Furthermore, interviews indicated that a data network project had been ongoing, as the budget allowed, over the preceding two years. The primary goal of the data network refresh project was to replace aging network equipment which had lost vendor support or had become obsolete. The following is an example of description of the data network, in addition to procurement drivers.

"The network is pretty old, about 10 years on average, and mostly end of life or end of support. We must juggle the budget, so we are replacing equipment when we get the money and try to do it as economically as possible. We get a lot of complaints about outages and performance, but if they gave us the money, we would replace it.” [DTCIONRF_NetworkUpgrade]

The data reveals a pattern of digital initiatives with only functional alignment for data network procurement. At no time did the data network team specifically mention any of the DX project initiatives. Neither did the network team mention general university mission requirements in relation of the data network which would indicate a relational direction or potential strategic alignment. 
It was also noted during direct observation that the CIO had mandated the existing end of life network equipment to be replaced with the newest version of the same models of equipment because monetary efficiencies were the primary determining factor for the procurement of the network. The decision to simply replace the existing data network with no consideration for the DX data dependencies of the data network or the overall current or future university mission irrespective of whether the subsequent projects were DX related or not could have rendered the data network insufficient to support any of these strategic requirements.

Similarly, the current state of network technology is such that the incumbent network vendor for the organization has technologies such as Digital Network Access (DNA) which comprise the incumbent OEM's core architecture for supporting DX initiatives.

No patterns with these terms were observed in the data except to note that ACI was attempted in the datacenter but not deployed due to the lack of staff skills. This would indicate that an attempt at strategic alignment was made, but it was not successful, and focus was realigned to support base functions of connectivity.

Likewise, recent strategic network technologies such as Software Defined Networking (SDN) or Intent Based Network (IBN) was expected. SDN and IBN are disruptive evolutions to traditional routing which elevates the network from traditionally rigid tactical business alignment of proving connectivity and not much else. SDN introduces capabilities which enable strategic network alignment by incorporating application requirements, in addition to business and policies and requirements into the operation of the network (Kim and Feamster, 2013). Similarly, IBN utilizes machine 
learning and automation to simplify the process of strategic alignment of the network (Butler, 2017). Without these terms the data network is being upgraded simply to the next iteration of existing tactically aligned architecture with no overt consideration of strategic business requirement in the network design or procurement process.

Likewise, a recurring pattern by the network team emerged in their answers to the questions in Table 8.

\begin{tabular}{|c|c|}
\hline \multicolumn{2}{|c|}{$\begin{array}{l}\text { Table } 8 \\
\text { PCS-1 Data network preparedness to support DX Sample Responses }\end{array}$} \\
\hline Question & Representative responses \\
\hline $\begin{array}{l}\text { Q9: Why did you conduct the most recent } \\
\text { network upgrade? }\end{array}$ & $\begin{array}{l}\text { "End of life" } \\
\text { "Old" } \\
\text { "End of support" } \\
\text { "Slow" }\end{array}$ \\
\hline $\begin{array}{l}\text { Q16: Is the data network ready for digital } \\
\text { transformation? Why or why not? }\end{array}$ & $\begin{array}{l}\text { "I don't think it can" } \\
\text { "I don't know" } \\
\text { "Leadership didn't think this through" }\end{array}$ \\
\hline
\end{tabular}

The observed pattern indicates that the data network has not undergone even a low-level transformation from functional to strategic alignment.

\section{Direct Observations}

The pilot case study revealed a pattern of relationships that support H1; however, there was no ability to validate relationships for the inverse, which would observe a positive relationship between a strategically aligned network and medium and high levels of DX project success and adoption. Likewise, there was no opportunity to observe if DX projects with a strategically aligned network could still achieve low DX adoption outcomes or DX project failure. The pilot case study also did not account for the effect of 
tangential influencers which can have a significant impact on DX project outcomes. It should be noted that these influencers are significant and include variables such as the effect of leadership, governance, culture, organizational maturity, organizational structure, and capabilities, to name only a few.

Considering the effect of influencers on DX outcomes as they relate to the data network was, please recall, outside the study's scope. What was within scope were the emergent patterns of deployment and alignment of the data network in relation to DX as illustrated in Figure 4. Figure 4 shows that there is an explicit lack of relationship between the data network refresh and the various DX projects. Therefore, where functionally aligned projects achieved a high of 'medium' as a DX outcome, this is an expectation of a network which has been designed with the function of connectivity in mind. 


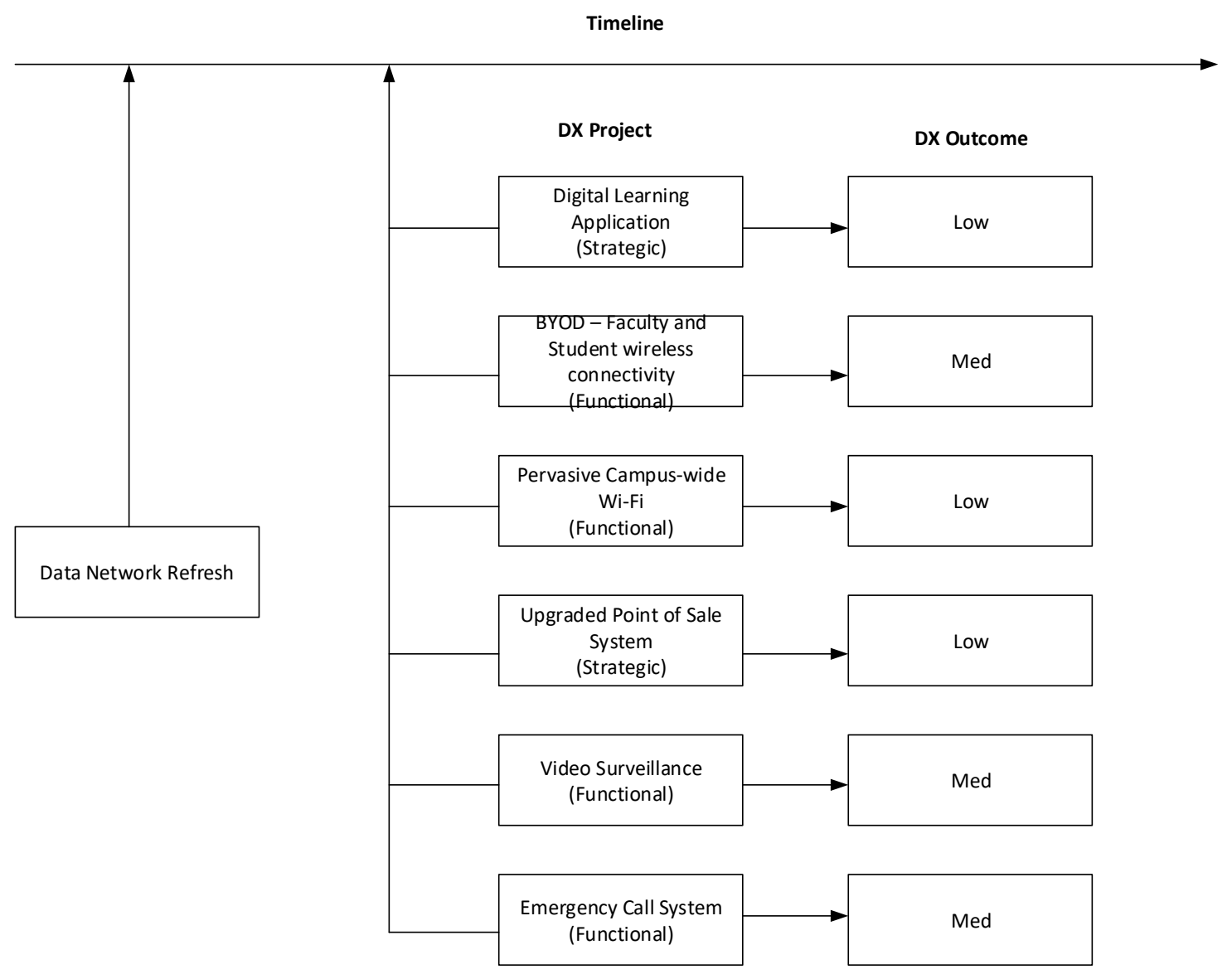

Figure 4. Relational View of PCS-1 Network-to-DX Projects

Furthermore, because price was a key factor in the selection of the data network equipment procurement, the equipment struggled to support the increased bandwidth requirements, an eventuality which led to the network being ill-suited for expanded functional requirements. Finally, with the functional and business teams working in distinct silos, all strategically aligned projects subsequently achieved low DX outcomes.

The DORS calculation produced a DX outcome level of .36, a medium-low score. Despite PCS-1 having multiple layers of executive oversight and sponsorship and a mature project management organization, they did not appear to affect the DX outcome, and further research should be conducted to better understand this phenomenon. The pilot 
case study did identify the effect of data network's contribution to digital adoption outcomes for PCS-1.

This relationship is a significant area for which the current literature is noticeably silent. This is true despite the shift in strategic fit of traditionally functional oriented technologies contemporarily reflective of data network technology. The shift in technology-to-business alignment of the data network is in line with the increasing capabilities and features of the technology and the digitization of business processes phenomena places new scrutiny on the rule functional IT purchasing decisions align with strategic organizational goals.

Existing quantitative research into DX has been invaluable in establishing causality in technology adoption and consumption models; however, the qualitative approach proposed in this research is an exploratory step in researching the effect of the data network on digital transformation outcomes. 


\title{
CHAPTER 5
}

\section{CASE STUDY 1 - A RETAIL FIRM IN THE U.S. HEARTLAND MAKES DIGITAL TRANSFORMATION THE HEART OF ITS OPERATIONS}

\author{
Initial Observations
}

MCS-1 is an 82-year-old, $\$ 8$ billion USD retailer with approximately 30,000 employees, and over 1800 stores spread throughout the continental United States. MCS1 's retail vertical is extremely competitive therefore participants in this space are balancing the need to streamline corporate, retail operations, and logistics, with the requirement to continually provide a positive shopping experience for its customers. Because the market in which MCS-1 operates is highly competitive, product margins are slim. MCS-1 cannot raise prices to increase profits because that would drive customers to their competitors, many of whom are even larger and have more resources available to them. As a result, MCS-1 must constantly increase operational efficiency at all levels, operating as leanly as possible while balancing efficiencies with increased responsiveness and service to their customers.

To this end, MCS-1 uses Infrastructure Led Disruption (ILD) to drive the majority of its firmwide operational initiatives, through digital transformation. ILD is a term originally coined in December 2019 by practitioner research and analytics firm Gartner, Inc. Kathrine Lord, a Vice President and analyst at Gartner, Inc. (2019) stated:

As businesses face increased pressure to lower operating costs, many Infrastructure and Operations (I\&O) leaders have been siloed into a tactical role rather than a strategic one - essentially, becoming custodians of legacy infrastructure. The result is stunted I\&O maturity over the past decade. However, I\&O leaders who harness the power of disruptive technologies, such as cloud and artificial intelligence, will discover new opportunities to serve as business innovators. I\&O leaders, who champion infrastructure-led disruption, are 
constantly looking for new ways to use technology to deliver business value, rather than just remaining reactive to stakeholder needs.

In 2016, the CEO at MCS-1 directed the company to include digital transformation as a cornerstone of its go to market strategy. The CEO positioned DX as a force multiplier to increase the organizations critical capabilities and as a competitive equalizer against its larger competitors which will increase the firms' competitive advantage and increase its market share. To enable this vision, the CEO relies on transforming the traditionally tactical posture of the role of CIO into that of a strategically relevant partner at the table in the execution of the overall corporate vision, mission, and go-to-market strategy.

With the CEO's vision in mind, interviews with the respective teams revealed that the various technology teams at MCS-1 found the task of transforming the organization's technological capabilities daunting and many were not convinced that such pervasive transformation was possible. Because the company historically focused primarily on the retail operations for its respective stores, its primary investments have been in traditional brick and mortar operations. Therefore, the focuses on traditional operations largely overlooked any strategic application of technology to achieve business requirements.

To achieve the CEO's mandate of infrastructure-led disruption through pervasive DX, it was deemed by the CIO that a thorough current state-of-the-technology assessment was necessary to ascertain the current functionality and capabilities of the existing systems. A six-month technology discovery project was subsequently sponsored and executed. The Project began in January 2017 and was concluded in May 2017. An application discovery project was conducted prior to the initiation of this case study; however, discovery during interviews with the various teams at MCS-1 deemed that the 
results of the study were directly applicable to the research being conducted, therefore necessitating inclusion of the project.

Interviews for this dissertation were conducted with the CIO, software application team members, and affected end users located at the headquarters site at which the case study was conducted. The interviews revealed that the applications, on which MCS-1 relied to conduct business operations, were a mix of self-contained (e.g., spreadsheets and word processor documents and files), home-grown proprietary applications. Interviews with end users revealed that the applications were prone to crashes and lockups, slow, and barely adequate to support the ever evolving and complex daily requirements of nationwide retail and business operations.

Interviews with the $\mathrm{CIO}$ also uncovered that custom programmed applications were not sustainable over the long term since they were reliant on the original programmers remaining with the firm for his or her entire career. Since that rarely happened, the firm had applications that could no longer be updated or customized since the programmer had subsequently left. As a result, these applications were frozen in time, depended upon for daily operations but not able to expand to support evolving operational requirements. The applications team stated that logistics systems required a high degree of two-way communications and extensive coordination with the retail stores, delivery vehicles, and warehouse staff. Furthermore, it was discovered that inventory often gets low, or items become depleted before they can be reordered causing items to be out-of-stock and customers forced to purchase the items from MCS-1's competitors. 
As expected, the retailer has an extensive and pervasive nationwide data network which connects not only its stores and point of sale (POS) systems, but also supports its corporate office connectivity requirements. However, interviews with the network team revealed that the data network was designed to provide basic functional connectivity between retail stores, datacenters, and headquarter locations.

Interviews and direct observations revealed that POS systems were reliant on the wide area network (WAN) for connectivity to the POS server which was located at MCS1's regional data center. However, the expanded footprint of the organizations' retail stores necessitated a patchwork of WAN circuits, many of which were unreliable and prone to outages. As a result, interviews with multiple teams revealed that retail operations were often interrupted when credit card processing could not be completed due to an outage of the WAN. Additionally, warehouse and logistics systems depended on the WAN also ceased to operate during outages.

Interviews with and observations of the network team revealed that the current data network had a significant negative impact on business operations. Random WAN outages could last minutes or hours. And to mitigate the downtime, which was detrimental to operations, MCS-1 utilized cellular network technology such as 4G as a backup system. However, the network team stated that many retail sites experienced WAN outages so frequently that those sites would exceed the $4 \mathrm{G}$ data allowance included in their plan which caused a dramatic increase in operational costs rather than offsetting the cost of reduced operations capabilities due to WAN outages.

Subsequent interviews with the CIO revealed that the discovery project identified several incumbent technology systems that needed upgrading or replacement. The CIO 
stated that he would not be able to meet the CEO's business initiatives based on the current state of technology. Therefore, initial targeted upgrades would include accounting, inventory, point-of-sale, and logistic systems, the implementation of a supplier focused Extranet for inventory management, and product logistics.

The CIO also stated that he wanted to go beyond merely upgrading traditional technologies on which retailers depend but wanted to deploy technology which would enable MCS-1 to understand how customers were interacting with their in-store displays and how the current store layouts affected customer engagement. Additionally, the CIO wanted to upgrade their legacy phone system to a unified communications architecture which would enable store managers to video conference with other regional stores, and corporate executives, to facilitate a high degree of store team interaction and coordination and to foster a greater sense of camaraderie. The CIO also expected to cut costs by reducing physical travel between stores for regional and national executives.

Direct observation and interviews therefore revealed the following pervasive DX projects for MCS-1 listed in Table 9. 


\begin{tabular}{|l|l|}
\hline \multicolumn{2}{|l|}{ Table 9 } \\
\hline \multicolumn{2}{|l|}{ MCS-1 DX Projects observed } \\
\hline Digital Transformation Technology & $\begin{array}{l}\text { Network technology dependency (by } \\
\text { order of precedence) }\end{array}$ \\
\hline $\begin{array}{l}\text { Project 1: SAP-based Accounting } \\
\text { Platform }\end{array}$ & $\begin{array}{l}\text { Local Area Network (LAN) } \\
\text { Wide Area Network (WAN) } \\
\text { Data Center Network (DCN) }\end{array}$ \\
\hline Project 2: SAP-Based Inventory System & $\begin{array}{l}\text { LAN } \\
\text { WAN }\end{array}$ \\
\hline Project 3: SAP-Based Logistics System & $\begin{array}{l}\text { LAN } \\
\text { WAN }\end{array}$ \\
\hline Project 4: Supplier Extranet & DCN \\
\hline $\begin{array}{l}\text { Project 5: WAN Backbone Upgrade } \\
\text { Project 6: Customer Tracking System } \\
\text { Project was Not Completed During the } \\
\text { Case Study Period) }\end{array}$ & WAN \\
\hline
\end{tabular}

Digital Transformation Technology Team Preparedness

Because MCS-1 has historically ignored innovative technology platforms as an integral part of its go-to-market strategy, the technical engineering, architecture, and support teams are lean compared to organizations of similar size. Derrick Price, a theoretical physicist, put forth a theory that postulates $50 \%$ of the work is done by the square root of the total number of people who participate in the work (Nicholls, 1988). As it were, only a handful of people contribute to $50 \%$ of the work. Since Price's law offers an empirical methodology for calculating ideal team size it was applied in this case. Therefore, if productivity decreases logarithmically from a minimum team size of 4 and a maximum team size of 108 , then it is considered optimal for a technical team size 
of 10 in most scenarios (Weaver, 2018). Whereas a team of 10 would yield an average productivity of $50 \%$ of a team of 4 , a team of 12 would yield productivity below $50 \%$.

MCS-1 had an average team size of 4. If Price's Law holds, then this implies that each member of the networking-team maintained productivity near $100 \%$ which meant that they were only functionally focused on daily operations and tech support. Direct observation and interviews reinforced this inference where it was commonly mentioned that there are not enough hours in the day, and they were always "putting out fires."

Additionally, the CIO and technology team managers stated that projects would take much longer than anticipated because there was little slack time in schedules to enable operations personnel to also participate effectively in technology projects. Finally, interviews revealed that here was little cross-functional cooperation between the data networking team and the applications teams.

During interviews, the CIO stated that he was aware that the technology teams were forced into a largely functional alignment due to lack of resources. He also stated that it was a key consideration to the lack of business alignment of the current network and applications systems. Furthermore, the CIO stated that he would augment his staff with consultants to facilitate the DX projects. Finally, the CIO stated that the current siloed operations of the various technical teams was not sustainable, and it was his goal to reassess how the respective teams, and the business at large, can benefit from crossfunctional operations.

\section{Project Kickoff and Stakeholder Inclusion}

Interviews with the CIO, data network team, applications development team, business operations team, and project managers revealed that designees from each 
respective team were appointed to represent the needs of the stakeholders. Each team worked internally with their stakeholders to identify the deficiencies of the incumbent technical and operational systems and identified the short (1 year), medium (3 year) and long term (+5 year) requirements that each would have to support.

The original kickoff meetings occurred prior to the start of the observation period. However, interviews were conducted retroactively to document the data network teams' gap analysis of the as-built technology and the process of gathering the requirements of the applications and business teams to ascertain the required outcomes of the subsequent network upgrade project. The result of the network team' gap analysis is detailed in Table 10. 


\begin{tabular}{|c|c|c|c|}
\hline \multicolumn{4}{|c|}{$\begin{array}{l}\text { Table } 10 \\
\text { MCS-1 Network Gap Analysis. }\end{array}$} \\
\hline Technology & As-built Network & Proposed Resolution & Posture \\
\hline $\begin{array}{l}\text { General Data } \\
\text { Network }\end{array}$ & Poor performance & $\begin{array}{l}\text { Increase per port and } \\
\text { uplink bandwidth using } \\
10 \mathrm{Gbps} \text { from current } 1 \\
\text { Gbps. }\end{array}$ & $\begin{array}{l}\text { Functional - } \\
\text { connectivity }\end{array}$ \\
\hline $\begin{array}{l}\text { General Data } \\
\text { Network }\end{array}$ & Frequent Outages & $\begin{array}{l}\text { Redesign network for } \\
\text { redundancy }\end{array}$ & $\begin{array}{l}\text { Functional - } \\
\text { connectivity }\end{array}$ \\
\hline $\begin{array}{l}\text { General Data } \\
\text { Network }\end{array}$ & $\begin{array}{l}\text { High operational and } \\
\text { configuration burden - } \\
\text { Slows store deployments }\end{array}$ & $\begin{array}{l}\text { Deploy application } \\
\text { recognition - } \\
\text { automation and } \\
\text { monitoring tools }\end{array}$ & Strategic - business \\
\hline $\begin{array}{l}\text { General Data } \\
\text { Network }\end{array}$ & $\begin{array}{l}\text { Overly complex - does not } \\
\text { support business } \\
\text { application deployments } \\
\end{array}$ & $\begin{array}{l}\text { Deploy software } \\
\text { defined networking } \\
\text { capabilities }\end{array}$ & Strategic - business \\
\hline $\begin{array}{l}\text { Wireless } \\
\text { Network }\end{array}$ & $\begin{array}{l}\text { Poor bandwidth and poor } \\
\text { coverage in stores }\end{array}$ & $\begin{array}{l}\text { Deploy Wi-Fi } 6 \\
(802.1 \mathrm{x}) \text { capable } \\
\text { wireless network }\end{array}$ & $\begin{array}{l}\text { Functional - } \\
\text { connectivity }\end{array}$ \\
\hline $\begin{array}{l}\text { Wireless } \\
\text { Network }\end{array}$ & $\begin{array}{l}\text { Does not support customer } \\
\text { tracking }\end{array}$ & $\begin{array}{l}\text { Deploy wireless } \\
\text { network which supports } \\
\text { end user location aware } \\
\text { smartphone } \\
\text { applications }\end{array}$ & Strategic - business \\
\hline $\begin{array}{l}\text { Telephone } \\
\text { System }\end{array}$ & $\begin{array}{l}\text { Current network does not } \\
\text { support Quality of Service } \\
\text { or application prioritization } \\
\text { for real-time applications. }\end{array}$ & $\begin{array}{l}\text { Deploy system wide } \\
\text { QoS and software } \\
\text { defined networking, }\end{array}$ & $\begin{array}{l}\text { Functional - } \\
\text { connectivity }\end{array}$ \\
\hline Collaboration & $\begin{array}{l}\text { Current network does not } \\
\text { support collaboration } \\
\text { applications to reduce } \\
\text { regional manager travel and } \\
\text { foster real time } \\
\text { collaboration between } \\
\text { stores }\end{array}$ & $\begin{array}{l}\text { Increase bandwidth, } \\
\text { deploy system wide } \\
\text { QoS and software } \\
\text { defined networking, }\end{array}$ & Strategic - business \\
\hline $\begin{array}{l}\text { Data Center } \\
\text { Network }\end{array}$ & $\begin{array}{l}\text { Data Center Network } \\
\text { (DCN) lacks redundancy, } \\
\text { security, and cannot support } \\
\text { requirements of new SAP } \\
\text { system }\end{array}$ & $\begin{array}{l}\text { Redesign DCN, } \\
\text { increase bandwidth and } \\
\text { redundancy. Introduce } \\
\text { software defined } \\
\text { networking } \\
\end{array}$ & Strategic - business \\
\hline $\begin{array}{l}\text { Data Center } \\
\text { Network / WAN }\end{array}$ & $\begin{array}{l}\text { Support migration from on- } \\
\text { premises data center to } \\
\text { cloud to reduce OPEX }\end{array}$ & $\begin{array}{l}\text { Redesign DCN to } \\
\text { include routing to } \\
\text { virtual private cloud }\end{array}$ & Strategic - business \\
\hline WAN & $\begin{array}{l}\text { Reduce store WAN outages } \\
\text { - save money on circuit } \\
\text { costs }\end{array}$ & $\begin{array}{l}\text { Deploy software } \\
\text { defined WAN (SD- } \\
\text { WAN) }\end{array}$ & Strategic - business \\
\hline
\end{tabular}


The respective teams agreed that the network upgrade and realignment project would precede all other technical and operations projects to reduce confusion and the complexity of attempting to manage multiple projects. The procurement criteria for the network equipment would be cross-checked against both the functional and strategic requirements uncovered in the data network, and subsequent application and operations team requirements documents.

\section{Outcomes and Conclusion}

During open coding, several themes emerged. Most notably was a notable lack of phrases such as "end of life." Even though the data network equipment approached the end of its useful lifespan (average of 7 to 10 years), this was NOT the key driver for the refresh. Additionally, all interviewees, irrespective of role, expressed concerns for the suitability of varying technology components, signifying an integrated relationship across project team members. For instance: interviews with the application teams often referenced the "network" or "datacenter." Similarly interviews with the data team referenced "application," "store operations," and "collaboration."

Finally, references to keywords that were not specifically technical but instead wholly business related (i.e., business, operations, and specific references to departments, customers, company mission, or specific applications) outnumbered keywords that were categorized as technically functional (i.e., bandwidth, connectivity.) by a ratio of 2:1.

Additionally, two thirds of technical team interviewees were able to paraphrase the corporate mission statement. Knowledge of the mission statement in technical teams is important to this research because it correlates the corporate mission has been relayed and internalized outside of business operations teams. This signifies an understanding of 
the overall corporate identity and mission which employees can use to tailor individual job roles to effectively support it (Ireland and Hitt, 1992). Understanding the corporate mission therefore signifies that DX projects resources understand the "why" in addition to the "how."

Direct observation revealed that the coordination and prioritization of the respective projects, and the acknowledgement that the incumbent systems were not enough to support current operational requirements signified a substantive shift to align technology with business mission. The willingness to reassess and modify operations and cross-functional team interactions signified a cultural shift which acted as a precursor to the subsequent business-centric strategic technology realignment and yielded the results as illustrated in Figure 5. 


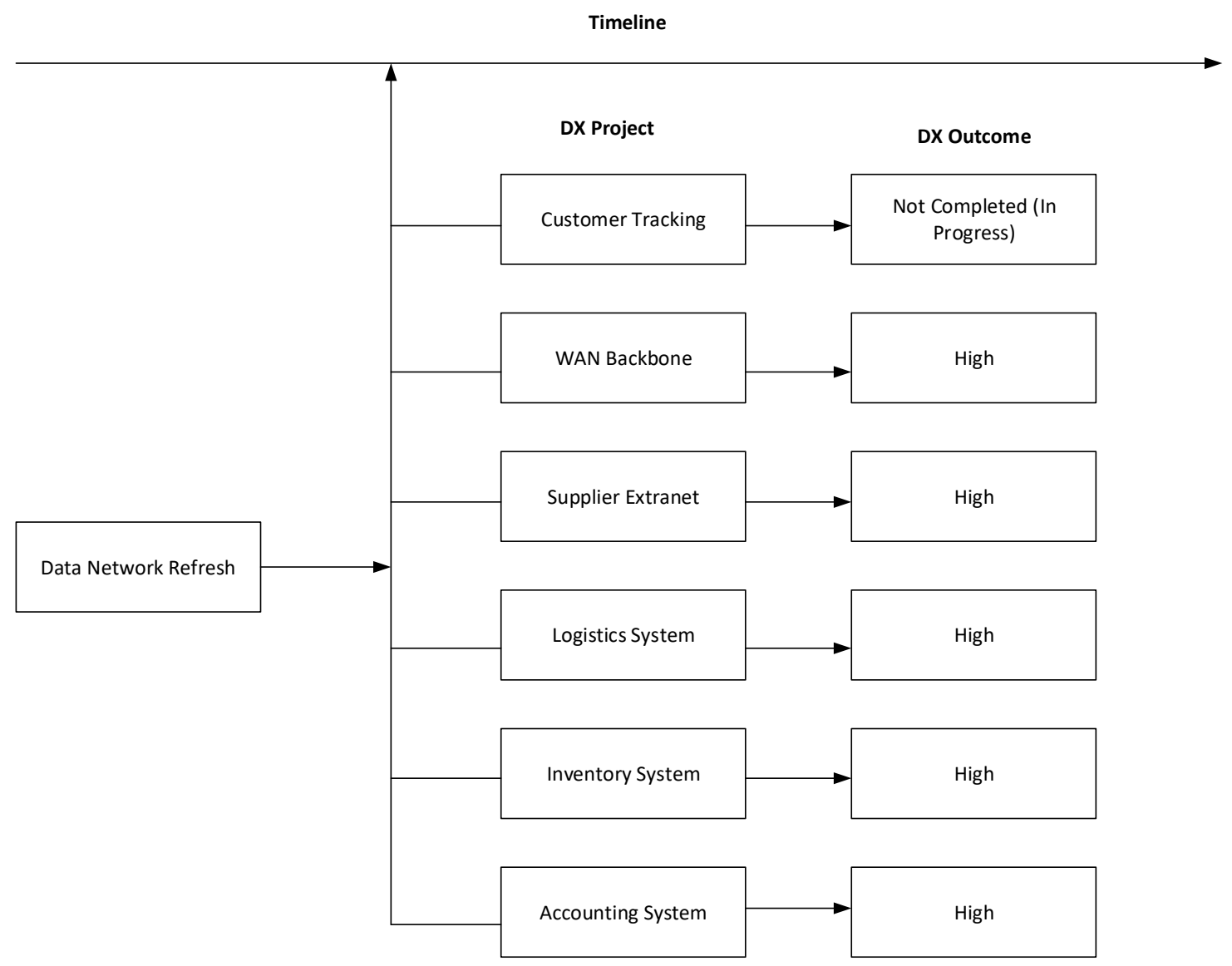

Figure 5. Relational View of MCS-1 Network-to-DX Projects

Because of the DX project's cross-functional interactions, the data network was specifically procured and aligned to the requirements of the DX projects. Additionally, the network, application, and business operations teams worked cooperatively to identify gaps in the data network which could adversely affect the overall DX project outcome.

The resulting data network alignment was inclusive of both functional and strategic goals, as was expected, but it was observed that a strong business case was subsequently drafted and submitted to the CFO to justify expenditure on more expensive network equipment to support the required operational functionality. Additionally, the key drivers defined in the business case for the network refresh were not highly 
functionally aligned, i.e., age of equipment, or refresh cycle. The attributes for performing the network refresh we tied to the DX-tied software application and overall DX project requirements, in addition to short, medium, and long-term corporate goals.

The new network was inclusive of next generation application-centric network technologies such as Wi-Fi 6 (802.1ax), Software Defined Branch, Software Defined WAN, Software Defined Datacenter, and Intent Based Networking. The respective technology architectures enabled the team to introduce automation, machine learning, and artificial intelligence, which was integral in reducing manual operation requirements, troubleshooting, operational cost, and verifying that the network is operating in a mode best suited to support the application and business requirements.

Interviews with end users revealed that the system achieved high adoption because of its high performance, integration with ancillary systems, and resiliency. Negative comments of the data network supported systems were focused on user interface and high learning curve. Delays in deployment due to programming issues were also observed. However, it was noted that two of the issues which caused delays were attributed to a misconfiguration in the network, which was due to a poorly defined application security requirement. Once this issue was resolved no further network related issues were observed.

The CIO and network teams also stated that there was a distinct reduction of shadow IT. Shadow IT is the use of unsanctioned by organization employees when they deem that the official IT tools are insufficient. This is often seen where the Wi-Fi has a poor signal; therefore, employees often bring in a Wi-Fi router from home. Or an application, such as a cloud-based word processor, runs poorly, thereby necessitating the 
use of another application chosen by the end-user, one, moreover, that is not sanctioned by the organization.

And while the respective projects are still ongoing, adoptions of all the target DX applications were $100 \%$, with no interviewees indicating no use of the new systems due to poor performance. Further interviews also indicated that the underlying functional aspects of the network have also been resolved with no interviewees causing patterns inclusive of terms such as "slow" or "poor" when responding to questions about their perception of the data network or the new DX related application.

Although high DX outcomes were observed in this case study, there are several moderators that contributed to the observed outcomes. First, the CIO identified the functional deficiencies of the existing data network infrastructure and tied the functional deficiencies to the inevitable strategic deficiencies which would have been detrimental to the overall DX project to continue in its current form.

Second, the CIO identified that the respective technical and operations teams were siloed and chose to drive collaboration between the respective teams to ensure that issues could be better identified. The subsequent cross-functional team collaboration ensured that gaps of the current network, and the required properties of the new data network to support the DX project were identified and mitigated. This increased collaboration were precursors to the subsequent procurement of the network equipment, which was key to driving resolution of current functional deficiencies and the alignment of strategic requirements of the data network.

Interviews revealed patterns that operational and technical personnel understood the business drivers and/or mission of the organization, an insight that presented an 
opportunity to apply strategic business requirements to data network infrastructure design and procurement. Additionally, interviewees applied this knowledge to ask critical questions about how technology would affect their roles and how their roles would affect the business. This signified an intrinsic cultural trait which was a strong moderator to the overall success of the DX projects.

These key observations identified important drivers to data network alignment and to the ultimate DX outcome. By comparison, there was a distinct lack of these moderators observed during the pilot study. Additionally, the actions of the CIO at MCS1 versus those at PCS-1 denotes both a disparity in leadership skills and in management style. However, the key drivers outside of the DX project technical requirements warrant further study to weigh their overall impact on DX outcome.

Finally, the DORS calculation for MSC-1 was .82, which indicates a high DX adoption outcome and is in line with the DX project adoption outcome directly observed at the conclusion of the project. 


\section{CHAPTER 6}

\section{CASE STUDY 2 - AN AUTOMOTIVE FIRM ATTEMPTS TO DRIVE PROFITS WITH DIGITAL TRANSFORMATION}

The Factors for Digitalization in the Automobile Market

MCS-2 is an automobile manufacturer founded in the early twentieth century and headquartered in Western Europe. MCS-2 has over 650,000 employees and 2018 revenues exceeding $\$ 270$ billion USD and operations in over 150 countries. The automobile market also highly competitive, with over 60 auto brands selling cars which target consumers at all levels of interest and needs, from those requiring basic and economical transportation through the ultra-luxury market segments. Current EBIT profit margins average approximately $6 \%$ (Hermes, 2017) and the cost of the average car in the United States is approximately $\$ 35,000$. Therefore, to increase profits automobile manufacturers are faced with three choices: increase sales price per car, increase the number of cars sold, or increase the profit per car sold.

It is well understood that the passenger automobile industry is not a growth industry. Statistica indicates that the number of passenger cars sold in the United States in 2019 (4.7 million) is less than the numbers of cars sold in 1951 (5.1 million), (Wagner, 2020). Additionally, the number of passenger cars sold globally is declining from a high of 71 million in 2016 to 64 million in 2019, which is less than the number of passenger cars sold in 2014 (66 million) (Wagner, 2020). Thus, the trends indicate that automobile manufacturers cannot depend on increasing the numbers of passenger cars sold to increase profits and must find other methods of increasing revenue. 
If the numbers of cars being sold is declining, then the option to increase prices is similarly challenging. Drew DeSilver and Pew Research calculated that the purchasing power for the average consumer in the United States has remained flat over the last 40 years. The 2018 dollar adjusted income for a worker in 1964 is $\$ 20.27$ per hour and the average per hour income in 2018 is \$22.65 (DeSilver, 2018).

However, the average price of a consumer automobile has risen from $\$ 23,900$ in 2008 to $\$ 39,000$ in 2019 representing a roughly $39 \%$ increase that far outpaces salary increases. To keep their automobile purchase affordable as a percentage of their salary, consumers are taking out longer term loans-between 72 and 84 months (Hecht, 2019). The increase in loan term converts directly to an increase in the period the consumer can purchase another automobile, thereby hinting at an inverse relationship between increased car prices and car sales.

The third option is to reduce the cost of each car sold, thereby realizing more profit per sale. The largest costs per car are raw materials (47\%), labor (21\%), advertising (7\%), and R\&D (6\%) (Kallstrom, 2015). The cost of raw materials such as steel and aluminum are driven largely by the market; automobile manufacturers have little control here except to favor building cars out of steel vs aluminum because steel is less expensive. However, steel is also heavier and heavier cars get fewer miles per gallon than their lighter counterparts. Therefore, manufacturers use lighter materials such as aluminum in their products to meet increasingly stringent fuel efficiency standards.

Cuts to R\&D are undertaken at an MCS-2's own detriment. Technologically advanced automobiles are increasingly status quo, even at base pricing levels and reflect the level of technology expectations that the average consumer experiences with other 
items with which they interface such as smartphones, computers, and tablets. Additionally, R\&D investment is needed as materials, materials, and capabilities of automobiles continue to evolve and must always be juxtaposed against the competition.

Cuts to advertising are possible; however, automobiles are increasingly a reflection of lifestyle. Automobile manufacturers not only have to project safety but also make the car appealing to the target demographic as an item which will be complementary to their respective lifestyles. As such, marketing becomes increasingly vital to drive sales. Therefore, the target for reducing cost per automobile is to reduce labor costs. Automobile manufacturers have already made heavy use of robots in the manufacturing process, and computers in the design process. However, there is a significant need to streamline the supply chain and internal operations processes to reduce cost. Because inefficiencies in these areas increase the time and decrease efficiency of logistics of supplies to factory locations, streamlining these areas can not only be cost effective, but also increase agility to respond to market forces driven by competitors and consumers alike. As such, MCS-2 undertook two multi-year DX projects to reduce per unit costs and enable it to increase revenue which can be subsequently invested into $\mathrm{R} \& \mathrm{D}$, which is vital for increased competitiveness.

The first project was the digital transformation of the supplier logistics infrastructure, referred to as the Supplier and Inventory Integration Network or SIIN². The goal of SIIN was to coordinate internal and external delivery of raw materials and parts more effectively and efficiently by enabling a 'just-in-time' delivery methodology for materials. Just in time delivery reduces both the variance in logistics and the delays in

\footnotetext{
${ }^{2}$ SIIN is not the real-world name of the project which has been anonymized for the purposes of this research.
} 
responding to market demand. These optimizations have a significant effect on smoothing the flow of materials to assembly plants, reducing the space required by not having too much raw materials delivered, and reducing the number of truck rolls by suppliers. MCS-2 can therefore establish more options to control the cost of acquiring raw materials. SIIN would ultimately enable the automation of the raw materials ordering process between MCS-2 and its suppliers to allow it to respond in real time to demand, manufacturing, delivery, and sales realities.

In addition to reducing the cost of raw materials, SIIN would enable MCS-2 with increased market agility by better delivering vehicles which are in higher demand to dealers more quickly, while also reducing production of vehicles in less demand in real time. This capability enables the delivery of automobiles to where they are needed, when they are needed, thus optimizing the end-product logistics process to the deal and subsequently, the end user. The resultant efficiencies thereby support faster realization of revenue for MCS-2.

An Internet of Things (IoT) project was also scheduled to add network connected sensors to all robots and automated systems which would, in turn, feed data to the SIIN system. Additionally, IoT would dive data to upcoming quality control systems and future high precision production control systems. IoT sensors would also be integrated into production automobiles to send real-time operational data to dealerships and to MCS-2. The resultant data would be part of MCS-2's future big data initiative to better understand driver trends and product performance in the real world. MCS would also send software updates to add new features and tune performance. 
The Best Laid Plans of Mice and Men Often Go Awry

Despite MCS-2's goals of cost reduction, increased profitability, and using IoT to incorporate unprecedented levels of intelligence into the production process and its automobile fleet, MCS-2 eventually postponed both SIIN and IOT projects indefinitely. A corresponding related data network upgrade was scheduled, but this too was delayed at the time of this writing and has now been reverted to the RFP phase. As a result, the observed level of adoption of the DX project was zero with a DORS of 0.0.

The two projects were conducted and simultaneously canceled before the inception of this case study; therefore, direct observation of the procurement and coordination of teams leading up to the project inception was not possible. However, unstructured interviews were conducted with two senior technology managers, two project managers, two leading members of the network team, and two members of the application team. The goal of the eight interviews was to derive emergent patterns, which could reveal overarching relationships which were integral to the DX failure, ex post facto. To support the research, one-hour interviews with each subject were conducted over three weeks, and there were no modifications of the interview guide despite the prior cancellation of the projects.

\section{Patterns Emerge from Interviews}

Interviews revealed the projects and associated network dependencies which are outlined in Table 11. 


\begin{tabular}{|l|l|}
\hline \multicolumn{2}{|l|}{ Table 11 } \\
\multicolumn{2}{|l|}{ MCS-2 DX Projects and Network Dependencies } \\
\hline Digital transformation technology & $\begin{array}{l}\text { Network technology dependency (by } \\
\text { order of precedence) }\end{array}$ \\
\hline Project 1: Supplier and Inventory & LAN \\
Integration Network (SIIN) & WAN \\
& DCN \\
\hline Project 2: Internet of Things (IoT) - Phase & LAN \\
1 & DCN \\
\hline
\end{tabular}

There was unilateral agreement by all interview subjects that the projects were cancelled due to pervasive poor application performance of both the SIIN and IoT technological components. The interviewees were not able to articulate the specific technical deficits affecting both projects since both postponed projects are still in the process of triage to define the way forward. However, all interviewees responded with common keywords "slow" and "connectivity" in respect to both projects, and neither project's attributable hardware nor software performed as intended.

All interviewees stated that a common original equipment manufacturer (OEM), which was critical for both the SIIN and IoT projects, indicated that the applications suffered from "timing out" at intermittent times during the day which would cause the application to lose connectivity and subsequently interrupt operations. The project managers stated that the projects in their current state were too disruptive to current operations to move forward. However, despite the current challenges, all interviewees stated that they believed that the projects would resume at an undefined later date once the technical and operational challenges were resolved. Interviewees referenced the current sunk costs in both projects at the time of this research were in excess of $\$ 10$ million in accumulated time and materials. 
Subsequent themes emerged during interviews which supported a distinct lack of understanding of the larger corporate mission, mission statement, or strategic goals of the company by non-management project resources. Emergent keywords such as "heads down" and "out of scope" by five of the seven interviewees indicated, when asked about the corporate mission statement, that they were primarily focused on the project and did not see business operations as being within the scope of their jobs.

Another emergent theme was the lack of the ability to articulate the component individual project deliverables by the project leaders. Additionally, despite codependences between the project resources for both projects, no non-management project resources could articulate the data network requirements.

Both network engineers also stated that they were not consulted regarding the suitability of the network prior to the start of either project despite the dependence of the data network. They responded with statements that included key themes such as "old" and "end of life" when asked about the current network state. Network Engineer 1 responded specifically that the network was not capable of supporting either project, or that "he told them this would happen." However, they were subsequently asked to join the troubleshooting process once the project teams identified performance issues with the network. What was clear, though, was that by the time the network engineers were consulted, the DX project was already in jeopardy. The interviews also revealed the project timelines as illustrated in Figure 6. 


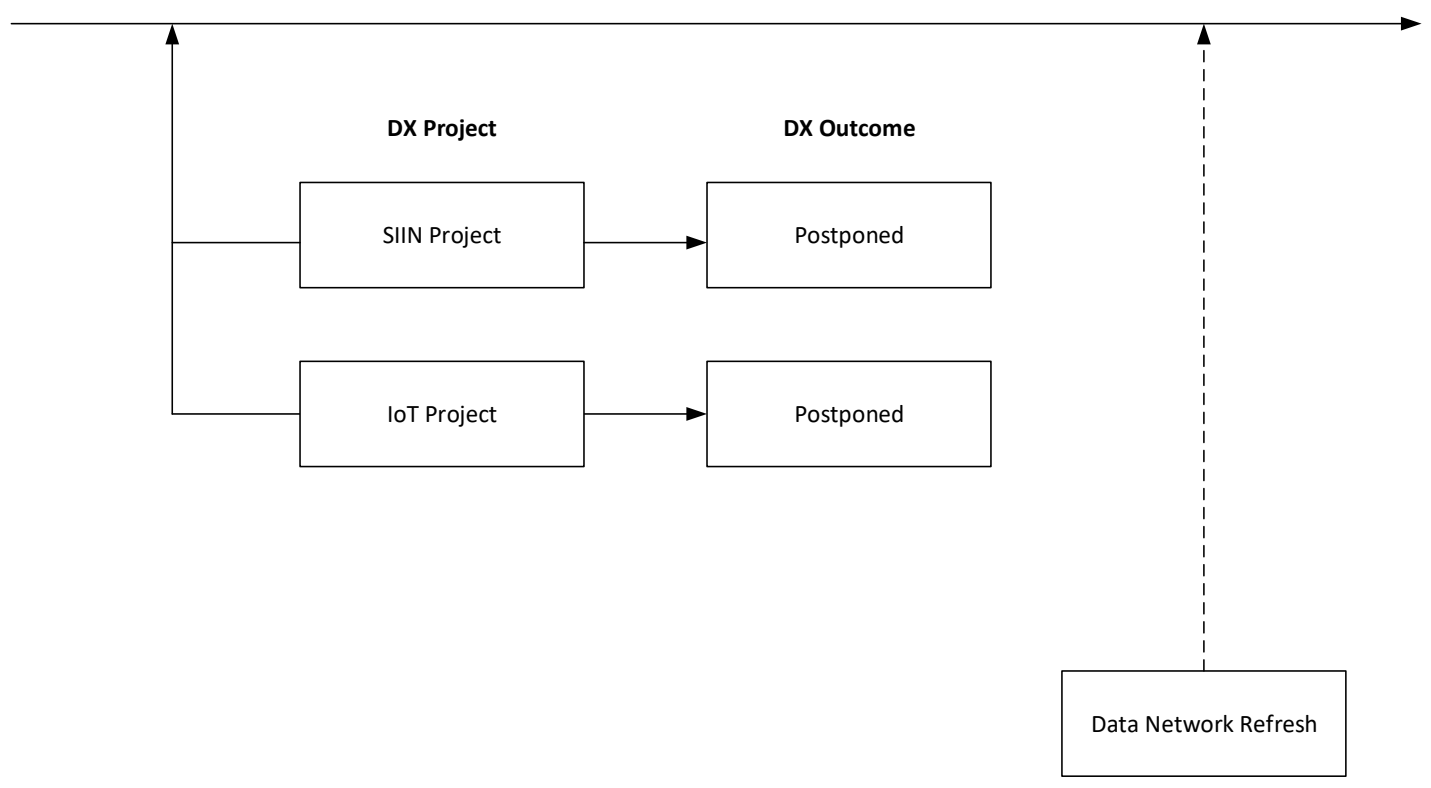

Figure 6. Relational View of MCS-2 Network-to-DX Projects.

Of note in Figure 6 is the explicit disassociation between the respective projects and the kickoff of the data network refresh project, ${ }^{3}$ yet to be determined at the time of this research. It was clear from the interviews that the network team did not feel the current network was adequate to support the project; however, the data network refresh was not associated or prioritized in relation to the SIIN or IoT projects.

\section{Digital Transformation Outcome and Conclusion}

Because the DX projects were suspended with none of the DX applications or operations having been adopted, the contingent DORS for all projects at MCS-2 was naturally 0.00 . No DX projects were successfully completed, and no end users were utilizing any of the DX applications or processes which were deliverables of either DX project.

Interviews revealed themes which implied that while there were sufficient skills and technical capabilities by project resources to complete either project, multiple

\footnotetext{
${ }^{3}$ Represented by the dashed line in Figure 5.
} 
operational, procedural, methodological, and technical deficiencies which would have strong moderation effects on the outcome of the respective projects. The total disconnection, and subsequent lack of mitigation, of an inadequate data network from the core projects was also noted; however, it would not be realistic to expect that the heavy influence of multiple moderators would have yielded medium or high DX outcome irrespective of the posture of the network.

Nonetheless, because the technical capabilities were adequate to complete the purely technical aspects of the SIIN, IoT, and network refresh projects, a DORS score would still be calculable at a future date. Follow up with MCS-2 once the project is completed would be useful to validate the research model, specifically where a functional network alignment causing low adoption can be realigned to achieve a strategic posture and thereby ultimately achieve medium or high DX adoption rates.

Finally, the effect of the lack of a suitable network infrastructure, which was a core technical dependency for both projects, cannot be discounted as a contributor to the initial project failures, especially, when interviews revealed common connectivity and performance issues with both projects. The lack of strategic alignment of the network was also disruptive to existing operations which utilized the same inadequate network infrastructure which suggests a common cause of disruption - the data network. 


\section{CHAPTER 7}

\section{CASE STUDY 3 - TOO BIG TO FAIL AT DIGITAL TRANSFORMATION}

MCS-3, a multibillion-dollar global manufacturer based in the United States, launched a pervasive global DX initiative in 2010, which was among the largest DX projects at the time.

The core of the MCS-3's digital transformation initiative is heavily reliant on IoT and big data technologies to transform its competitive capabilities across all of its verticals. The ambitious DX goal was to transform the firm from a legacy manufacturing company to that of a technology company ${ }^{4}$ that relies on disruptive and forward leaning technologies as key market differentiators. MCS-3 aspired to effectively deliver a global manufacturing internetwork which would connect internal and external systems to suppliers, manufacturing, and network connected end user products deployed in the field. And while its DX initiative was expansive enough to involve MCS-3's many internal and external systems, the focus of the case study was narrowed to capture the level of digital adoption of its core IoT and connected systems DX initiatives.

The requirement for MCS-3's DX projects were distilled into the following key initiatives: (1) connect "things"; (2) pull data from "things"; (3) store data derived from "things"; (4) analyze data from "things"; and (5) use the data from "things" to drive disruptive innovation. These goals would thereby lead to a market advantage versus the competition because of the immense amount and diverse breadth of data available to MCS-3 as a global manufacturer of a highly diverse portfolio of "things." Therefore, a key component of the DX initiative is heavily reliant on connectivity, e.g., the data

\footnotetext{
${ }^{4}$ If DX project had succeeded, MCS-3 would have been disqualified as a subject of research.
} 
network, for success. Table 12 illustrates the DX technology and the requisite data network dependencies.

\begin{tabular}{|c|c|}
\hline $\begin{array}{l}\text { Table } 12 \\
\text { MCS-3 DX Project and Network Dep }\end{array}$ & \\
\hline Digital transformation technology & $\begin{array}{l}\text { Network technology dependency (by } \\
\text { order of precedence) }\end{array}$ \\
\hline Project 1: Big Data & $\begin{array}{l}\text { LAN } \\
\text { WAN } \\
\text { DCN - Internal / Cloud }\end{array}$ \\
\hline Project 2: Internet of Things (IoT) & $\begin{array}{l}\text { LAN } \\
\text { DCN } \\
\text { WAN }\end{array}$ \\
\hline
\end{tabular}

By 2015, MCS-3 discovered that the DX technology and operational integration was too ambitious and disruptive to existing operations; therefore, it divested the DX operation as a separate entity within the larger enterprise. As a result, the new DX startup unit would develop and provide digital innovation to the larger corporate entity and develop new technology projects for consumption in the marketplace. However, by 2017, MCS-3's new digital entity had failed to accomplish many of the goals set in its original charter, and digital adoption of the project outputs were largely non-existent whether internally, externally, or as new technology products.

At the time of this research (2019-2020), MCS-3's digital unit had reassessed its original mission which supported a charter focused on feeding DX innovation into the larger entity and pivoted on transforming into a software technology company, a factor which would have disqualified it from inclusion in this paper. However, the networkreliant entity which existed from 2015 through 2018 is still of interest for inclusion because it included a significant DX related network and datacenter expenditure to support the IoT and big data initiatives. And while it was not possible to observe the 
activities of that time period, there was significant interest in understanding the themes that would emerge during unstructured interviews, which may portend a relationship between the status of the larger DX venture and the data network.

Interviews were conducted with two senior executives and three senior network architects who were directly involved in the DX project and offer cradle-to-grave visibility into the project. The senior executives were project sponsors with a combined tenure of over fourth years MCS-3. One of the senior executives is still employed with MCS-3 and the other left the firm subsequent to the interviews. Both network architects were employees of MCS-3 from the inception of the project and are currently employed with MCS-3; however, they are working in other capacities and no longer in direct support of any projects which are recognized as being strategically transformative.

Because there had been a significant and visible failure of the DX initiative, it was important to understand how the respective projects were positioned on the timeline in relation to any network refresh projects. Figure 7 illustrates the timelines gleaned from the interviews.

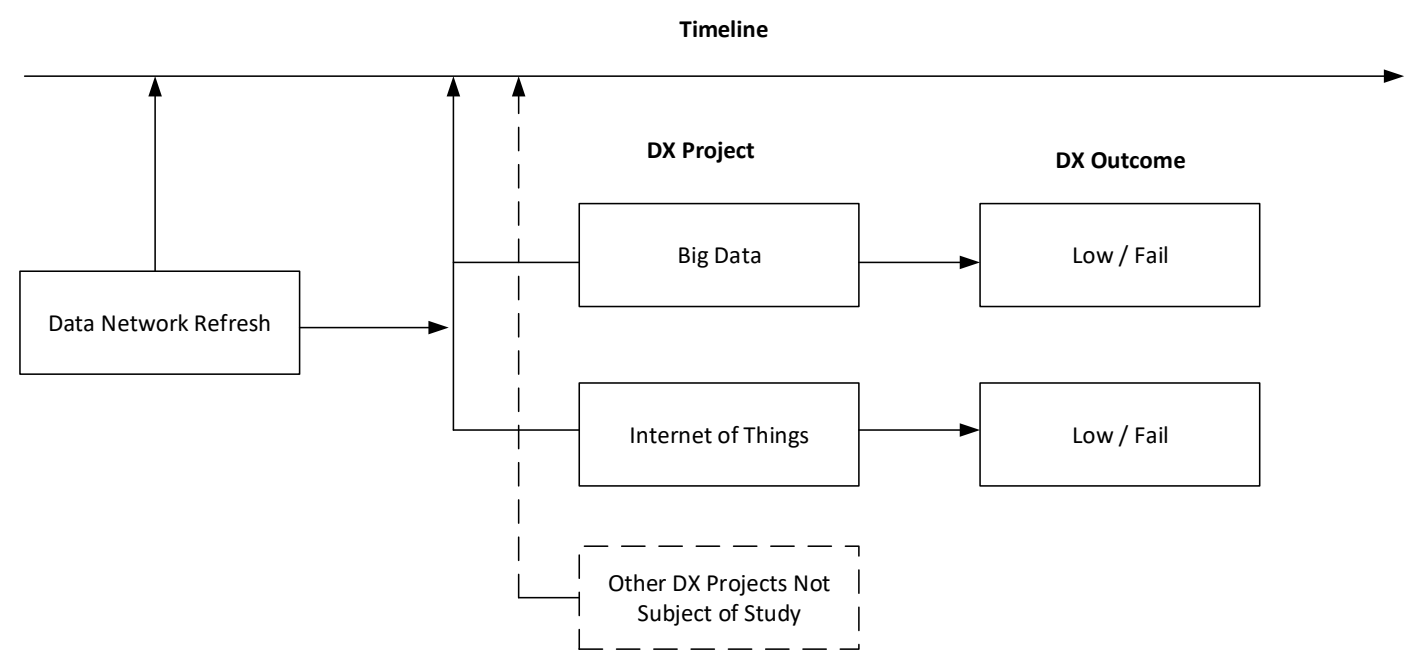

Figure 7. Relational View of MCS-3 Network-to-DX Projects 
Although there were other DX projects in progress, the Big Data and IoT projects were similar enough to other projects to be a representative analog of all DX projects which lead to low or failed adoption. During interviews both network architects stated that the data network was refreshed cyclically every five-to-seven years; therefore, the network usually did not reach an end-of-life stage. Where the term "end-of-life" was used, it was in relation to not being a factor at MCS-3, not as a property of the network equipment. Furthermore, the network architects stated that the existing data network was in middle of a cyclical refresh when the DX projects began and the overall requirements for the network refresh were outside of the specifications of the DX projects. However, network architect interviewees also stated that they consulted which implied that the capabilities of the network were a key consideration of the separate DX projects.

All interviewees articulated the gaps of the performance and capacity of the data network at the inception of the DX projects and all interviewees similarly articulated bandwidth and technology properties they felt were required to successfully support the DX projects. While the network architects articulated the network gaps at length, as is expected of human resources with their skill and experience levels, the executives could also articulate the gaps in the data network at a high level.

Additionally, themes referencing the most significant gaps and concerns with the data network such as bandwidth, resiliency, and redundancy emerged. In interviews, the executives conveyed concern that the network should be robust enough to support continued expected corporate growth throughout MCS-3's global footprint, irrespective of the existence of a global DX projects. The interviewees stated that gaps, which were identified directly in relation to the DX projects, were congruent with the overall 
corporate network gaps and the keywords thereby overlapped to illustrate the similarities in the respective themes.

However, the network architects stated that there was a specific focus on and concern for the legacy on-premises and cloud datacenter networking capabilities. Because the global scale of the projects generated petabytes of data to be transported, stored, and analyzed, there would be a critical focus, with an appropriate budget allocated, to increase the global datacenter network capabilities. Likewise, because data is harvested and transported from anywhere in the world, there would be significant investments in increasing the bandwidth and throughput capabilities of the WAN.

With the data network, specifically bandwidth, cloud and datacenter networks, emerging as a central theme; and the unilateral use of the word "inadequate" emerging most frequently across all interviewees, a central theme emerged in respect to the lack of ability of MCS-3's data networks strategic suitability to support big data and IoT devices capability to communicate and transport data adequately. However, these terms were not used in relation to a triage of the DX failure, but rather to identify a gap which was addressed continually throughout the term of the DX project.

Further interviews revealed that the investments into remediating the gaps in the network infrastructure required a large scale which was comparable to that of a cloud service provider such as Microsoft's Azure, Google's Cloud, or Amazon's Web Services. The global scale of the data network requirements was so vast that MCS-3 would scale to the size of a cloud provider and it was never in the project charter to achieve such scale. Additionally, the cost to scale to that size would necessitate that the cost to the internal and external users and business units be far greater for users to use the MCS-3 backbone 
and data center than to simply use a cloud providers existing backbone as part of the DX projects.

Therefore, the interviews revealed that the scale and ambition of the project outgrew the capability of MCS-3 to support it at either at the financial, operational, or technical levels. Additionally, the DX project that spun off into its own semi-autonomous entity had profit and loss ( $\mathrm{P}$ and $\mathrm{L}$ ) obligations, which required the $\mathrm{DX}$ project units to operate as an existing business and not as a startup venture. Where a startup is given latitude to generate short term loss in exchange for long term gain, and established business is expected to be profitable now. Obviously, the fledgling DX spinoff could not meet the rigors of the latter.

\section{Digital Transformation Outcome and Conclusion}

The interviews revealed a compelling narrative of a highly skilled technology team, at a firm with significant financial, operational, and personnel resources, with clear operational and project requirements which were highly aligned with business requirements at all levels. Such a firm would check all the proverbial boxes and a high DX outcome is expected.

Furthermore, the cross-functional communications and interworking processes were more mature than any of the subjects studied thus far. The status quo for the posture of the data network was strategically aligned. That is to say, the networking and business teams continually collaborated to ensure that the network was aligned to support the global business requirements. Additionally, end-of-life network components was not a consideration since MCS-3's refresh cycle was as short as five years. 
For the purposes of network alignment with the DX projects, the network was purposefully built to support requirement for data intensive applications on a global scale, which presents an operational and financial obstacle for MCS-3. The global network requirements for both projects exacted a large toll in terms of finances and personnel resources. Additionally, it was extremely disruptive to the existing operational of the firm. MCS-3's DX project grew so large that the company became a cloud provider unto itself. However, cloud providers build their network infrastructure with an eye on extremely high efficiency ratios per unit of data. Conversely enterprises typically spend more per unit of data because their data consumption is much lower than cloud providers. As a result, the scope of the DX projects was so disruptive, ambitious, and expensive that the requirements of the supporting data network inadvertently transformed the nature of the DX spinoff from the intended purpose of a provider of digital software services to a cloud service provider.

Despite the serious challenges and realignment of DX priorities, the DORS for internal DX adoption is still medium/high at .68. However, as expected, external adoption was low with a DORS of .19. Both DORS were combined to produce a mean DORS of .43 , which represents a medium DX outcome.

The DX project achieved medium-high adoption internally and the DX processes have met or exceeded many of the firm's internal transformative technological aspirations. It did so by delivering new operational tools and more efficient processes, inclusive of global manufacturing and logistical economies of scale. Conversely, the separate business entity has low external adoption because the cost and scale of the network, and other systems, required to sustain the separate DX organization and 
products made fully adoption impractical. While the digital organization exists at a modified smaller scale, the DORS calculation was conducted using the original project charter and not the modified scale of the revised organizational size.

Interviews also supported significant influencing factors which had adverse effects on both external and internal DX project outcomes. The moderators which had the biggest impact were financial; therefore, even though there was a successful strategic DX project to network alignment, which initially yielded high DORS above .80 for both internal and external projects, they were not financially or operationally sustainable. 


\section{CHAPTER 8}

\section{CASE STUDY 4 - AN AIRLINE STRUGGLES TO GAIN MARKET ALTITUDE WITH DX}

MCS-4, an airline founded in the mid-1990s, experiences the same business challenges as the other 10 major airlines based in in the United States and shares the similar patchwork of high and low financial performance over time with other airlines. However, with its focus on fliers whose primary consideration is cost, MCS-4 is tasked with building a desirable brand without the frills and extended travel options, such as priority economy, business, and first-class cabins, factors which most airlines depend on for increased revenue. And while MCS-4 has adopted the same a la carte service add-ons now widely adopted by the majority of airlines, MCS-4 must rely on operational efficiency and a conservative fiscal policy to remain profitable.

To construct a complete picture of challenges which MCS-4 faces, five days of direct observation were granted with the technical operations teams. Additionally, interviews were conducted with a senior executive in charge of line operations, two senior technology managers, a consulting project manager who has been directly involved in technology projects at the airline for over seven years, and two senior data network architects. With direct access to resources covering both the business and technology challenges of the airline a complete picture of key digital transformation drivers took shape.

As is true of most U.S. air carriers, many of whom are no longer in business, MCS-4 has experienced losses that can be counted in the billions of U.S. dollars. It has 
gone through mergers, layoffs, bankruptcies, restructuring, and a pervasive culture of cost cutting necessary to stay solvent in a hyper-competitive industry. However, while MCS-4 has always been challenged with keeping costs under control to exact the highest per seat revenue possible, this has had a tremendous negative effect on its operations and customer service rankings. By 2016 the airline had one of the worse on-time departure and arrival records and one of the highest instances of lost and misplaced baggage. Its customer satisfaction ratings were one of the lowest in the industry and the airline became the airline of last resort where low cost made choosing MCS-3 a necessity.

Despite MCS-4's low customer satisfaction ratings, companies like Southwest Airlines have proven that a low-cost model does not mean that good service and customer loyalty are necessarily tied to low cost and profitability. Southwest Airlines consistently maintains one of the highest on-time performance records to complement its customer satisfaction rankings, which consistently rank amount the top three among U.S. airlines. Therefore, MCS-4 realized that its negative customer satisfaction trajectory was not sustainable: they are losing unhappy customers to their competitors, and these unhappy customers often only return to fly MCS-4 again only as a last resort.

Likewise, MCS4's extensive operational inefficiencies are not only an inconvenience for customers - significantly contributing to its low customer satisfaction ratings - but they also have a domino effect on operations which causes aircraft and crews to become de-synchronized. Equipment and crews consistently being out of position eventually becomes a financial drain on the firm because they affect operations system wide causing subsequent flights to be late or even cancelled. 
With the negative operational and fiscal trajectory being unsustainable for the future viability of the airline, the executives effected a pervasive modernization plan to address its operational deficits. The plan included modernizing the aircraft fleet and upgrading front and backend computer systems, along with the associated technologies supporting them. The cornerstone of the technological modernization was digital transformation which would, enable new levels of operational efficiencies that would have a positive impact on all areas of MCS-4 and ultimately have a positive effect on customer satisfaction, and eventually the bottom line. The executive team at MCS-4 saw modernization as vital to the survival of the airline and failure could mean that the airline would eventually cease to exist.

Before the precise technological projects, and subsequent costs could be identified, MCS-4 executives stated that an end-to-end technology system inventory and gap analysis was required. The inherent historical neglect of the current systems meant that MCS-4's technology leadership teams simply did not know what they did not know, despite knowing that the primary target of years of cost cutting had often been technology upgrades. MCS-4 did not historically view technology as a strategic tool; therefore, technology investments were constantly deferred until the balance sheet indicated greater levels of profitability which would fund the much-needed technology refreshes and updates.

Unfortunately, profitable times for MCS-4 which would enable technology upgrades were rare. Thus, MCS-4's current state was such that most of the technological infrastructure was a mixture of end-of-life equipment, decades old software application systems which incorporated customized, and unsustainable, home-grown updates and 
patches. Additionally, customer service and call center systems were antiquated and lacked seamless integration into related reservation and Internet systems.

Critical aircraft ground operations systems did not fare any better. It is standard parlance in the airline industry to identify aircraft support systems as above-the-wing and below-the-wing and both systems were identified as inadequate to support MCS's technology-enabled streamlined operational goals. Above the wing systems are inclusive of electronic flight bags for the pilot, point of sale (POS) systems for use by the flight attendants and in-flight entertainment technologies such as Wi-Fi and inflight entertainment systems (IFE). To this end pilots were still using paper charts and other manual tools. Many pilots have resorted to using their own laptops and tablets to augment their needs for online charts and other requirements. ${ }^{5}$ Flight attendants had to accept cash due to lack of POS systems and only a few newly acquired aircraft had Wi-Fi systems which are needed for passenger entertainment, but also to connect inflight POS and electronic flight bag systems. Likewise, below-the-wing systems were equally lacking. MCS-4 had an antiquated baggage tracking system, which was the primary reason that it had one of the highest lost bag rates in the industry.

There was also no Wi-Fi connectivity in employee crew rooms or airport general gate areas; therefore, flight crews and gate agents were tethered to podiums or needed to access wired terminals to check in passengers, check their schedules, and the crew checkin and check-out process with crew scheduling at the end of their flights had to be transacted via phone or via a wired computer. This also meant that the crew scheduling

\footnotetext{
${ }^{5}$ An example of shadow IT in play.
} 
department lacked the resources necessary to support complex and flexible aircraft operations; late arrivals caused crew conflicts and, subsequently, further flight delays.

Finally, the data network was either end of life or low-end equipment which was procured to support the comparatively light data transmission requirements and limited dispersion, of older reservations and operation systems. However, executives reviewed successful DX case studies which indicated that scale of such DX requirements included connected airports, equipment, aircraft, and enabling crew with tablets and smartphone connectivity via wireless while in airports and in the air. Therefore, the amount of data transversing the network would grow exponentially.

Additionally, modern data-network crew and aircraft scheduling systems consider dozens, if not hundreds, of datapoints from weather and winds aloft, to historic airport taxi times to properly calculate flight schedules. The lack of these technological capabilities was a key reason why MCS-4 experienced significant challenges in improving on-time arrival and departure. With the business drivers clearly identified, over a dozen DX projects were scheduled over a three to five-year period. The case study focused only on the six digital transformation projects which were directly reliant on the data network as listed in Table 13. 


\begin{tabular}{|l|l|}
\hline \multicolumn{2}{|l|}{ Table 13 } \\
MCS-4 Data Network Reliant DX Projects \\
\hline Digital transformation technology & $\begin{array}{l}\text { Network technology dependency (by } \\
\text { order of precedence) }\end{array}$ \\
\hline $\begin{array}{l}\text { Project 1: New and Updated Customer } \\
\text { Service and Reservation Systems }\end{array}$ & $\begin{array}{l}\text { LAN } \\
\text { WAN } \\
\text { DCN }\end{array}$ \\
\hline $\begin{array}{l}\text { Project 2: New Voice over IP Call Center } \\
\text { with Interconnectivity to New Reservation } \\
\text { Systems }\end{array}$ & LAN \\
\hline Project 3: New and Updated Crew \\
Scheduling and Aircraft Management & DCN \\
Systems & LAN \\
\hline Project 4: Baggage Tracking with RFID & DCN \\
Bag Tags and Readers & LAN \\
\hline Project 5: Wi-Fi Connectivity in & WAN \\
Employee and Crew Areas, and Ramp & LAN \\
Connectivity for Above / Below Wing & WAN \\
Operations & \\
\hline Project 5: Systemwide Data Network & LAN \\
Upgrade (LAN, WLAN, WAN, DCN) & WAN \\
\hline
\end{tabular}

Interviews revealed that when the original scope of DX projects were identified, it was common knowledge that the ability of the data network to support the projects was doubtful. Data network gaps were identified through an exhaustive technology gap analysis, which originally did not include the data network, but executives and project sponsors subsequently added the network to the analysis when adding WLAN (Wireless LAN) network capabilities to ramp and crew areas forced the project sponsors to assess end-to-end supporting network systems on which the WLAN would rely. As each layer (access, distribution, core) of the network was accessed, it was found that constant neglect, due to budget cuts and funding of more pressing fiscal priorities, meant that some data network equipment was almost 13 years old. 
Additionally, many of the network switches and routers were currently at $60 \%$ traffic utilization. Therefore the $30-50 \%$ projected increase in data traffic in some areas could increase data through the network infrastructure past $80 \%$ utilization, which is recognized as the maximum traffic saturation level before there is significant network performance degradation.

None of the interviewees stated that MCS-4 had used technology strategically. Likewise, all interviewees stated that the company historically saw technology as a cost center, leading technology to receive minimal investment and even then, only when it was a necessity, i.e., end of life or end of support equipment. Both technology executives also stated that the data network received a lower budget than the application and software system units. The data network budget for equipment refreshes, prior to the inception of the DX projects, were often less than \$1 million USD. Completion of a gap analysis was integral, hence, for MCS-4 to make an investment of over \$7 million to upgrade and "future-proof" the data network at all levels.

Corporate and technology executives also identified the requirement to complete the data network project prior to the start of any data network-reliant upgrades. Interviewee TE2 framed the identification of the data network as being integral to the overall DX project success as a "happy accident."

Other technology projects, not reliant on the data network, were started under separate timelines; however, they were all managed under a single technology upgrade program. As such, interviews were sufficient to construct a timeline and relationship map to for respective DX projects and subsequent outcomes as illustrated in Figure 8. 


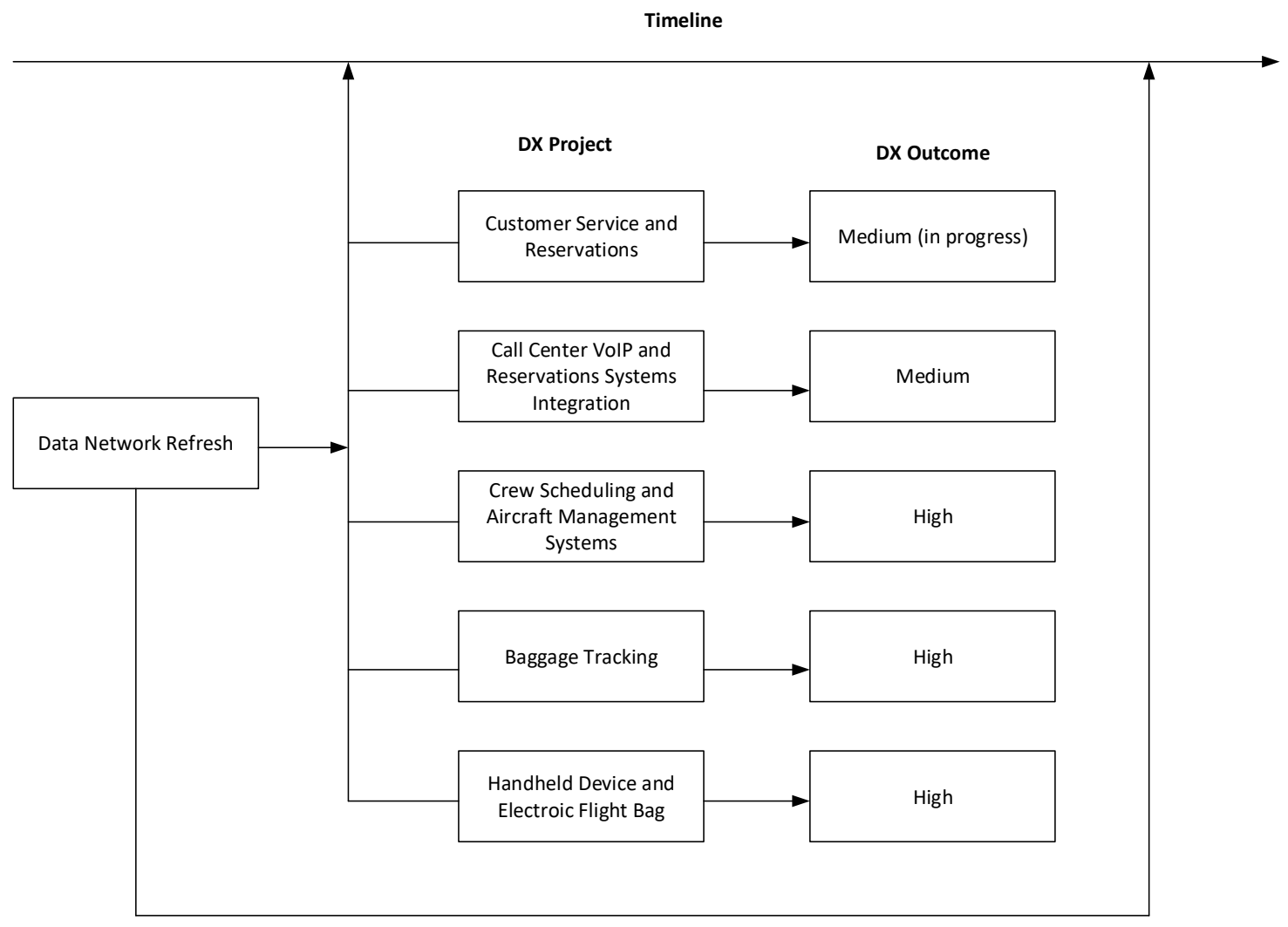

Figure 8. Relational View of MCS-4 Network-to-DX Projects

Of note is the data network project being bisected into DX project and subsequent non-DX project alignments. The initial network investment supported the DX project, with further network budget allocated for continued data network upgrades that are not causally related to the current projects. The network architects stated that nascent network technologies such as Software Defined Networking and cloud network infrastructures were on their strategic roadmap. All interviewees also stated that the expected outcomes of the future projects were greater operational efficiency for managing the network as well as greater agility for bringing new applications, connected services, or even bringing new airport locations online. 


\section{Digital Transformation Outcome and Conclusion}

The final case study extended common themes common throughout this research: (1) MCS-4 is increasingly reliant on the efficiencies of technology transformation of processes at all levels of operations; (2) key technologies supporting the firms DX are network centric; and (3) DX technologies drive new levels of data about the business and customers, which can be analyzed and used to support competitive advantages (i.e., big data). Although the requirement of a data network to support current and future DX goals was identified largely by accident, the realization and subsequent mitigation of data network infrastructure was sufficient to effectively mitigate years of neglecting the data network.

The total calculated DORS for MCS-4 is .77, which results in a medium/high digital adoption outcome despite historically low technology investments and a comparatively immature technology strategy as compared to other firms that were studied.

MCS-4's leadership did exhibit a willingness to make radical directional changes as it relates to its historic attitude towards technology. Subject TM1, a technology manager, mentioned that she was able to convey the network investment requirements of the DX program to the financial and operational leadership team and received little pushback regarding the financial investments. Both network architects stated that frequent data network outages had brought the inadequacy of the data network to the attention of executive leadership years ago; therefore, it is safe to postulate that MCS-4's leadership felt that it was not possible to put off the network upgrades any further, especially if it meant success or failure of the overall DX program. 


\section{CHAPTER 9}

\section{COMBINATORIAL OBSERVATIONS AND RESULTS}

The current research is an in-depth exploratory observation of DX projects currently in progress or recently having been recently completed to observe the impact of a previously unstudied variable, the data network, on DX projects.

As a reminder, the outcomes of DX projects were quantified by measuring the resultant post-project DX adoption by building a scoring mechanism. This was required because at the time of this research no quantifiable method of measuring DX outcomes existed. The scoring mechanism, Digital Outcome Raw Score (DORS), was constructed and applied to the adoption outcomes of all the researched DX projects to provide precision measurement of the specific level of project deliverables and DX adoption achieved at the formal conclusion of the DX project. For the purposes of aligning DORS to DX, the outcome range is between 0 and 1 with the intermediate fractions representing DX outcomes:

$$
\begin{aligned}
& 1.0-.8=\text { High } \\
& .79-.60=\text { Medium-High } \\
& .59-.40=\text { Medium } \\
& .39-.20=\text { Medium-Low } \\
& .19-.01=\text { Low }
\end{aligned}
$$

A simple fractional measurement between 0 to 1 scale was determined to afford sufficient precision, yet simplicity, for determining a score to DX outcomes. 
To this end, the DORS calculations, which are inclusive of DX project status, DX adoption status, and resultant calculations for each respective pilot and multiple case study participants are detailed in Table 14. 


\begin{tabular}{|c|c|c|c|c|c|c|c|c|c|c|c|c|}
\hline \multicolumn{13}{|c|}{\begin{tabular}{|l|} 
Table 14 \\
DORS Outcome Calculation Detail
\end{tabular}} \\
\hline \multirow[t]{2}{*}{ Variable } & \multirow{2}{*}{\multicolumn{2}{|c|}{$\begin{array}{c}\text { PCS-1 } \\
\text { Status and Scores }\end{array}$}} & \multirow{2}{*}{\multicolumn{2}{|c|}{$\begin{array}{c}\text { MCS-1 } \\
\text { Status and Scores }\end{array}$}} & \multirow{2}{*}{\multicolumn{2}{|c|}{$\begin{array}{c}\text { MCS-2 } \\
\text { Status and Scores }\end{array}$}} & \multicolumn{4}{|c|}{ MCS-3 } & \multirow{2}{*}{\multicolumn{2}{|c|}{$\begin{array}{c}\text { MCS-4 } \\
\text { Status and Score }\end{array}$}} \\
\hline & & & & & & & \multicolumn{2}{|c|}{$\begin{array}{c}\text { Status and Scores } \\
\text { External }\end{array}$} & \multicolumn{2}{|c|}{$\begin{array}{c}\text { Status and Scores } \\
\text { Internal }\end{array}$} & & \\
\hline \multicolumn{13}{|c|}{ DX Projects Outcomes } \\
\hline Project 1 & Partial & .05 & Full & 1 & Postponed & Null & Partial & .5 & Full & 1 & Full & 1 \\
\hline Project 2 & Partial & .05 & Full & 1 & Postponed & Null & Partial & .5 & Full & 1 & Full & 1 \\
\hline Project 3 & Partial & .05 & Full & 1 & - & - & - & - & - & - & Full & 1 \\
\hline Project 4 & Partial & .05 & Full & 1 & - & - & - & - & - & - & Full & 1 \\
\hline Project 5 & Partial & .05 & Full & 1 & - & - & - & - & - & - & Full & 1 \\
\hline Project 6 & Partial & .05 & Pending & Null & - & - & - & - & - & - & - & - \\
\hline \multicolumn{13}{|c|}{ DX $_{x}$ Calculation Outputs } \\
\hline $\mathbf{D X}_{d}$ & & 6 & & 5 & & Null & & 2 & & 2 & & 5 \\
\hline $\mathbf{D X}_{\mathbf{a}}$ & & 3 & & 5 & & Null & & 1 & & 2 & & 5 \\
\hline $\mathbf{D X}_{\mathbf{x}}$ & & .5 & & 1 & & Null & & .5 & & 1 & & $\mathbf{1}$ \\
\hline \multicolumn{13}{|c|}{ DX Projects Population and Adoption } \\
\hline Project 1 & $1500 / 3500$ & .83 & $850 / 1100$ & .77 & $\begin{array}{c}\text { Not } \\
\text { Calculated } \\
\end{array}$ & Null & $135 / 500$ & .30 & $350 / 500$ & .65 & $305 / 550$ & .61 \\
\hline Project 2 & $20000 / 25000$ & .80 & $22000 / 25000$ & .88 & $\begin{array}{c}\text { Not } \\
\text { Calculated }\end{array}$ & Null & $52 / 250$ & .45 & $137 / 250$ & .70 & $120 / 200$ & .60 \\
\hline Project 3 & $12500 / 25000$ & .50 & $300 / 350$ & .86 & - & - & - & - & - & - & $323 / 575$ & .86 \\
\hline Project 4 & $25 / 50$ (sites) & .50 & $120 / 150$ & .80 & - & - & - & - & - & - & $594 / 675$ & .88 \\
\hline Project 5 & $600 / 700$ & .85 & $95 / 120$ & .79 & - & - & - & - & - & - & $484 / 550$ & .88 \\
\hline Project 6 & $2800 / 3500$ & .80 & - & - & - & - & - & - & - & - & - & - \\
\hline \multicolumn{13}{|c|}{ DXy Calculation Outputs } \\
\hline $\mathbf{D X}_{\mathbf{p}}$ & & 1 & & 1 & & Null & & 1 & & 1 & & 1 \\
\hline $\mathbf{D X}_{\mathbf{u}}$ & & .71 & & .82 & & Null & & .37 & & .68 & & .77 \\
\hline $\mathbf{D X}_{\mathbf{y}}$ & & .71 & & .82 & & Null & & .37 & & .68 & & .77 \\
\hline \multicolumn{13}{|c|}{ DX $X_{0}$ Digital Output Raw Score } \\
\hline $\mathbf{D X}_{\mathbf{0}}$ & & .36 & & .82 & & Null & & .19 & & .68 & & .77 \\
\hline
\end{tabular}


As detailed earlier the calculations for DORS was conducted using the following

formula.

$$
\begin{aligned}
& \left(\frac{\mathrm{DX}_{a}}{\mathrm{DX}_{d}}\right)=\mathrm{DX}_{x} \\
& \left(\frac{\mathrm{DX}_{u}}{\mathrm{DX}_{p}}\right)=\mathrm{DX}_{o}
\end{aligned}
$$

Referencing Table 14 one of three values were affixed to produce the score assigned to the project deliverable. A value of 1 signifies that the deliverable was completed fully. A general value of .5 signifies that the project deliverable was partially completed. It should be noted that a general value, and not a value signifying the actual level of project deliverable completion, was assigned because the general value was sufficient to provide an overall score sufficient for describing the phenomena without adding undue complexity to the calculation. A value 0 was assigned if the organization failed to complete the project deliverable. Finally, a null value was assigned if the organization postponed the project deliverable past the observation period of the respective multiple case study.

To describe the total end-user population affected by the DX project, a maximum value of 1 was used. The end-user population using each DX project deliverable was measured against the total potential end-user population and is represented as a fraction of 1 . 
The DORS are then summarized in Table 15 and assigned an outcome range as previously mentioned above. The determination of the functional or strategic alignment of the data network was derived from the observations in the multiple case studies.

\begin{tabular}{|c|c|c|c|}
\hline \multicolumn{3}{|l|}{ Table 15 } \\
Comparative Multiple Case Study Results \\
\hline Case & DORS & DX Outcome Level & $\begin{array}{c}\text { Data Network } \\
\text { Alignment to DX } \\
\text { Project? }\end{array}$ \\
\hline PCS-1 & .36 & Medium-Low & No \\
\hline MCS-1 & .82 & High & Yes \\
\hline MCS-2 & $.00^{1}$ & Postponed & No \\
\hline MCS-3 & $.43^{2}$ & Medium & Yes \\
\hline MCS-4 & .77 & Medium-High & \\
\hline
\end{tabular}

The data suggests that a loose causal relationship exists between the inclusion of the data network as a key component of supporting applicable DX projects and business requirements, e.g., strategic alignment. Subjects who included network alignment in support of business and DX requirements prior to the start of the DX project achieved high or medium-high DORS versus subjects who did not, those that received low or medium-low scores. Additionally, Figure 9 illustrates the range of scores from all observed subjects.

The results of the case study therefore infer a hypo-dissertation that there is a positive relationship between DX projects conducted by strategically aligning the data network to support the DX project. 
Furthermore, the research supported and validated the proposed DORS

calculation as a tool sufficient for describing organizational DX outcomes. When DORS is applied to the DX project outcomes, it appeared to provide an accurate measurement of DX project results which were directly observed. Thus, DORS enables firms to affix precision to the observation of DX project outcomes versus a simple pass-fail assignment of DX outcomes, which was the status quo prior to this research.

Additionally, the interview results revealed specific keywords which uncovered patterns which were illustrative of a strategic alignment of the data network versus a tactical alignment as detailed in Table 16.

\begin{tabular}{|c|c|l|c|}
\hline \multicolumn{4}{|l|}{ Table 16 } \\
Keywords indicating Strategic or Tactical Data Network Alignment. \\
\hline Case & DORS & \multicolumn{1}{|c|}{ Predominant Keywords and Phrases } & $\begin{array}{c}\text { Strategically } \\
\text { Aligned } \\
\text { Data } \\
\text { Network? }\end{array}$ \\
\hline PCS-1 & .36 & $\begin{array}{l}\text { End-of-Life, Bandwidth, Budget, Heads- } \\
\text { down, Outages, Poor Performance, Expired, } \\
\text { Siloed, Compromise, Unreliable, Old, Low } \\
\text { Morale }\end{array}$ & No \\
\hline MCS-1 & .83 & $\begin{array}{l}\text { Mission, Team, Redundant, Goal, } \\
\text { Applications, Architecture, Security, High- } \\
\text { Speed, Resilient, Budget, Refresh }\end{array}$ & Yes \\
\hline MCS-2 & .00 & $\begin{array}{l}\text { End-of-Life, Slow, Blame, Applications, } \\
\text { Silo, Understaffed, Performance, Busy } \\
\text { Compromise, Overworked }\end{array}$ & No \\
\hline MCS-3 & $.43^{1}$ & $\begin{array}{l}\text { Culture, Global, High-speed, Cloud, } \\
\text { Applications, Team, Project, Software- } \\
\text { Defined, Refresh, Architecture, Technology }\end{array}$ & Yes \\
\hline MCS-4 & .77 & $\begin{array}{l}\text { End-of-Life, Capacity, Technology, Team, } \\
\text { Project, Refresh, Budget, Neglect, } \\
\text { Architecture, Support, Compromise }\end{array}$ & Yes \\
\hline
\end{tabular}


Patterns emerged at firms with a historically tactical data network, a situation that indicated that they were primarily inward facing. These patterns emerged as common themes where tactically aligned teams were preoccupied with internal network related operational tasks focusing on troubleshooting network related issues as the core of their activities. Tactically aligned teams were also very reactive and found themselves responding to issues not covered by users outside of the network unit. As a result, when the DX project was overlaid on the tactically aligned network, the network could not support the increase in workload and data requirements and the studied teams were ill equipped to support the DX project since they lacked the cross functional consultation between the network, business, and project teams.

Conversely, patterns emerged at firms with a historically strategic network alignment which implied an outward facing posture. Strategically aligned teams were focused on supporting the larger corporate mission, working in cross-functional teams, and participating as part of a matrixed project team. Patterns at strategically aligned teams indicated increased proactivity in identifying weak areas of the network. This was because cross functional working groups enabled the network to be tuned to support a diverse range of business initiatives and organizational user profiles. The strategically aligned teams also frequently referenced culture as a positive aspect versus the tactically aligned teams that frequently referred to lack of culture or a reactionary culture.

However, case study MCS-3 demonstrated that despite an initial functional alignment of the data network, it is possible to pivot to a strategic realignment. MCS-3 initially experienced a low DORS however, both the technical, project, and executive teams were worked closely together to identify the process and technical gaps which 
contributed to the original low DX outcomes. It was observed that involvement by all stakeholders, in addition to the willingness to implement a cross functional methodological approach to reassessing the original DX project deficits were key. Therefore MCS-3 identified the key dependencies and deficiencies which were lacking from the first DX project attempt and used the outputs from those observations to address newly deployed DX systems which were performing poorly. Table 16 shows that the keywords associated with the final DORS was in line with the expectations for a strategically aligned data network where the predominant theme was focused on the larger DX project and business rather than on functional tasks such as end-of life dates and hardware refresh cycles. 


\section{CHAPTER 10}

\section{DISCUSSION, IMPLICATIONS, RECOMMENDATIONS FOR FURTHER STUDY, AND CONCLUSION}

This research explored the relationship of the data network to DX outcomes, with an emphasis on measuring the subsequent DX project outcomes with precision. As the research progressed it was clear that outcomes were tied to a human dynamic which was indicative of capabilities that extended well beyond the capabilities of the data network equipment which was procured.

Outcomes of DX projects are of contemporary interest because, at the time of this research, most DX projects fail wherein billions of dollars are wasted. The multiple case study approach afforded the opportunity to isolate a significant dependent variable intrinsic to most DX projects and measure the outcomes with more precision (Yin, 2014). To this end, the exploratory nature of the research required the formulation of a quantifiable method of measuring the outcomes of digital transformation to successfully compete this research. The result of that formulation requirement is DORS, a transformational metric which was successfully utilized throughout the research. This metric, we believe, is a major contribution of the dissertation.

This exploratory research provided qualitative prima facia evidence inferring a direct relationship between the strategic or tactical posture of the data network and various levels of DX outcomes. However, the research uncovered widely varied moderators which also have a strong direct effect on DX outcomes. These moderators that range from team dynamics and corporate culture to resource and fiscal constraints. While the research infers that data network posture has a critical effect on DX outcomes, 
these projects are ultimately reliant on the humans implementing them. Therefore, the results and relationships posited in this paper are undoubtedly codependent on other variables with a significant effect on the DX outcome. The significant potential effect of moderators is a key reason why DORS is intended as a raw score. Therefore, DORS calculates the raw, and direct effect of the data network on the DX outcome but does not factor moderators into the equation.

Although multiple case studies offer significant depth and breadth of data collection though direct observation of poorly understood themes (Yin, 2014), case studies are exploratory in nature and can only loosely infer causal evidence of effects on a phenomenon being studied. Additionally, the research does suggest relationships, but it can offer no definitive proof of causation. However, the dissertation does offer insights valuable for building a complete framework in the future, one that could be used for further qualitative/quantitative analyses to verify the causative effects uncovered in this research. It hence also offers directions for research which can more strongly establish inference of causation.

Furthermore, the research reinforced the innate difficulty in engaging in organizational case study research in that organizations are loath to reveal information that could conceivably generate negative press for the firm, tip their hand regarding proprietary initiatives, or violate financial regulations which could infer forward looking directions or reveal insider information. All firms in this case study insisted on strict anonymity and required a full review of the case study research in which they were referenced. Furthermore, while participant reviews of case study data do reinforce construct validity (Yin, 2014), the firms did require the removal of any data with which 
they felt uncomfortable, even under the guarantee of anonymity. As a result, primary research tools such as DORS are challenging to apply since they require either direct observation or survey data to complete.

Finally, the research found no evidence that the preeminent technology adoption models discussed in this paper are insufficient for explaining the DX phenomenon. However, as applications continue to move to the cloud, the performance of the network becomes another factor influencing technology adoption and perceived usefulness. The current adoption models were identified as being application-centric; however, the reliance is shifting from the computing power of the end user to the end user connection to the cloud. Therefore, the data in this research can offer directions for modifications of the current models to account for the data network and its effect on end user perception, which in turn drives technology adoption. The research also hints at future directions to fill the gaps in research identified in this paper in regard to organizational adoption of technology.

Despite its limitations, the outputs from this research provides incremental advancement and useful qualitative primary data of a nascent area of digital transformation research. The intent of this paper was that it would become a document which both academic and practitioner researchers could use to further validate the data in this paper and utilize DORS to assist companies in increasing the probabilities for success in their DX journey. 


\section{REFERENCES CITED}

Agarwal, R., and Lucas Jr, H. C. (2005). The information systems identity crisis: Focusing on high-visibility and high-impact research. MIS quarterly, 381-398.

Agarwal, R., and Prasad, J. (1998). A conceptual and operational definition of personal innovativeness in the domain of information technology. Information systems research, 9(2), 204-215.

Agarwal, R., and Prasad, J. (1998). The antecedents and consequents of user perceptions in 9information technology adoption. Decision support systems, 22(1), 15-29.

Ajzen, I. (1991). The theory of planned behavior. Organizational behavior and human decision processes, 50(2), 179-211.

Andal-Ancion, A., Cartwright, P. A., and Yip, G. S. (2003). The digital transformation of traditional business. MIT Sloan Management Review, 44(4), 34-41.

Annarhecht. (2019, October 22). Car prices are increasing-here's how that can hurt Americans. Retrieved January 15, 2020, from https://www.cnbc.com/2019/10/22/car-prices-are-rapidly-increasing-heres-whythats-bad-for-americans.html

Bacon, C. J. (1992). The use of decision criteria in selecting information systems/technology investments. MIS quarterly, 335-353.

Bakos, J. Y., and Treacy, M. E. (1986). Information technology and corporate strategy: a research perspective. MIS quarterly, 107-119.

Bandura, A. (1989). Human agency in social cognitive theory. American psychologist, 44(9), 1175.

Baxter, P., and Jack, S. (2008). Qualitative case study methodology: Study design and implementation for novice researchers. The qualitative report, 13(4), 544-559.

Berman, S. J. (2012). Digital transformation: opportunities to create new business models. Strategy and Leadership, 40(2), 16-24.

Beshley, M., Pryslupskyi, A., Panchenko, O., and Beshley, H. (2019, July). SDN/Cloud Solutions for Intent-Based Networking. In 2019 3rd International Conference on Advanced Information and Communications Technologies (AICT) (pp. 22-25). IEEE.

Bharadwaj, A. S. (2000). A resource-based perspective on information technology capability and firm performance: an empirical investigation. MIS quarterly, 169196. 
Bharadwaj, A., El Sawy, O., Pavlou, P., and Venkatraman, N. (2013). Digital business strategy: toward a next generation of insights.

Bhattacherjee, A., and Premkumar, G. (2004). Understanding Changes in Belief and Attitude toward Information Technology Usage: A Theoretical Model and Longitudinal Test. MIS Quarterly, 28(2), 229. doi:10.2307/25148634

Burton-Jones, A., McLean, E. R., and Monod, E. (2015). Theoretical perspectives in IS research: from variance and process to conceptual latitude and conceptual fit. European journal of information systems, 24(6), 664-679.

Butler, Brandon. "What is intent-based networking?" Network World, 23 June 2017. Gale Academic OneFile, https://link-galecom.libproxy.temple.edu/apps/doc/A496597041/AONE?u=temple_mainandsid=A ONEandxid=a45b6a78. Accessed 27 July 2020.

Campbell, D. T. (1975). III. "Degrees of freedom" and the case study. Comparative political studies, 8(2), 178-193.

Campbell, D. T., and Stanley, J. C. (2015). Experimental and quasi-experimental designs for research. Ravenio Books.

Carr, N. G. (2003). IT doesn't matter. Educause Review, 38, 24-38.

Carter Jr., F.J., Jambulingam, T., Gupta, V.K., Melone, N., 2001. Technological innovations: a framework for communicating diffusion effects. Information and Management 38 (5), 277-287.

Châlons, C., and Dufft, N. (2017). The role of IT as an enabler of digital transformation. in the drivers of digital transformation (pp. 13-22). Springer, Cham.

Chandler, A.D. Strategy and Structure: Chapters in the History of the Industrial Enterprise. Cambridge, MA: MIT Press, 1962.

Chiyangwa, T. B., and Alexander, P. T. (2016). Rapidly co-evolving technology adoption and diffusion models. Telematics and Informatics, 33(1), 56-76.

Christie, M., Rowe, P., Perry, C., and Chamard, J. (2000, June). Implementation of realism in case study research methodology. In International Council for Small Business, Annual Conference (pp. 1-21).

Croteau, A. M., and Bergeron, F. (2001). An information technology trilogy: business strategy, technological deployment, and organizational performance. The journal of strategic information systems, 10(2), 77-99. 
Cusimano, A. (2013). Making connections between CFO and CIO. Strategic Finance, 94(11), 45.

DeSilver, D. (2018, August 7). For most Americans, real wages have barely budged for decades. Retrieved January 10, 2020, from https://www.pewresearch.org/facttank/2018/08/07/for-most-us-workers-real-wages-have-barely-budged-fordecades/

Doody, Owen and M Doody, Catriona. (2015). Conducting a pilot study: Case study of a novice researcher. British Journal of Nursing. 24. 1074-1078.

10.12968/bjon.2015.24.21.1074.

Davern, M. J., and Kauffman, R. J. (2000). Discovering potential and realizing value from information technology investments. Journal of Management Information Systems, 16(4), 121-143.

Davis, F. D., Bagozzi, R. P., and Warshaw, P. R. (1989). User acceptance of computer technology: a comparison of two theoretical models. Management science, 35(8), 982-1003.

Demirkan, H., Spohrer, J. C., and Welser, J. J. (2016). Digital innovation and strategic transformation. IT Professional, 18(6), 14-18.

Drnevich, P. L., and Croson, D. C. (2013). Information technology and business-level strategy: Toward an integrated theoretical perspective. Mis Quarterly, 37(2), 483509.

Eisenhardt, K. M. (1989). Building theories from case study research. Academy of management review, 14(4), 532-550.

Flyvbjerg, B., and Budzier, A. (2011). Why Your IT Project May Be Riskier than You Think. SSRN Electronic Journal. doi:10.2139/ssrn.2229735.

Furr, N., \& Shipilov, A. (2019). Digital doesn't have to be disruptive: the best results can come from adaptation rather than reinvention. Harvard Business Review, 97(4), 94-104.

Gallivan, M. J. (2001). Organizational adoption and assimilation of complex technological innovations: development and application of a new framework. ACM Sigmis Database, 32(3), 51-85.

Gartner Says Infrastructure-Led Disruption Will Drive Business Innovation. (2019, November 11). Retrieved January 7, 2020, from https://www.gartner.com/en/newsroom/press-releases/2019-12-11-gartner-saysinfrastructure-led-disruption-will-drive-business-innovation 
Goodhue, D. L., and Thompson, R. L. (1995). Task-technology fit and individual performance. MIS quarterly, 213-236.

Gindy, N., Morcos, M., Cerit, B., and Hodgson, A. (2008). Strategic technology alignment road mapping STAR $®$ aligning RandD investments with business needs. International Journal of Computer Integrated Manufacturing, 21(8), 957970.

Goerzig, D., and Bauernhansl, T. (2018). Enterprise architectures for the digital transformation in small and medium-sized enterprises. Procedia CIRP, 67, 540545.

Guang-dong, W. (2013). THE RELATIONSHIP BETWEEN PROJECT TEAM DYNAMIC FEATURE, CONFLICT DIMENSION AND PROJECT SUCCESS-AN EMPIRICAL RESEARCH FROM SHANGHAI, CHINA. Pakistan Journal of Statistics, 29(6).

Gurbaxani, V., and Dunkle, D. (2019). Gearing Up for Successful Digital Transformation. MIS Quarterly Executive, 18(3).

Hameed, M. A., Counsell, S., and Swift, S. (2012). A conceptual model for the process of IT innovation adoption in organizations. Journal of Engineering and Technology Management, 29(3), 358-390.

Hess, T., Matt, C., Benlian, A., and Wiesböck, F. (2016). Options for Formulating a Digital Transformation Strategy. MIS Quarterly Executive, 15(2).

Henderson, J. C., and Venkatraman, H. (1993). Strategic alignment: Leveraging information technology for transforming organizations. IBM systems journal, 32(1), 472-484.

Henderson, J. C., and Venkatraman, H. (1999). Strategic alignment: Leveraging information technology for transforming organizations. IBM systems journal, $38(2.3), 472-484$.

H. Kim and N. Feamster, "Improving network management with software defined networking," in IEEE Communications Magazine, vol. 51, no. 2, pp. 114-119, February 2013, doi: 10.1109/MCOM.2013.6461195.

Ireland, R. Duane, and Michael A. Hirc. "Mission Statements: Importance, Challenge, and Recommendations for Development." Business Horizons, vol. 35, no. 3, 1992, pp. 34-42., doi:10.1016/0007-6813(92)90067-j.

ISO, I. (1994). IEC 7498-1: 1994 information technology-open systems interconnectionbasic reference model: The basic model. ISO standard ISO/IEC, 7498-1. 
Jin, B. H., and Li, Y. M. (2012). Analysis of emerging technology adoption for the digital content market. Information Technology and Management, 13(3), 149-165.

Jöhnk, J., Oesterle, S., Ollig, P., and Riedel, L. N. (2020). The Complexity of Digital Transformation-Conceptualizing Multiple Concurrent Initiatives. In 15th International Conference on Wirtschaftsinformatik.

Kallstrom, H., Raw materials - the biggest cost driver in the auto industry. (2015, February 10). Retrieved January 10, 2020, from https://marketrealist.com/2015/02/raw-materials-biggest-cost-driver-autoindustry/

Kauffman, R. J., and Weill, P. (1989). An evaluative framework for research on the performance effects of information technology investment.

Karahanna, E., Straub, D. W., and Chervany, N. L. (1999). Information technology adoption across time: a cross-sectional comparison of pre-adoption and postadoption beliefs. MIS quarterly, 183-213.

Khanagha, S., Volberda, H., Sidhu, J., and Oshri, I. (2013). Management innovation and adoption of emerging technologies: The case of cloud computing. European Management Review, 10(1), 51-67.

Kotusev, S. (2016, April). The critical scrutiny of TOGAF | IT management | Subject ... Retrieved December 10, 2017, from http://www.bing.com/cr?IG=CA6DBA712D5B43F4A05CB745E12BD53DandCI $\mathrm{D}=054 \mathrm{D} 21 \mathrm{~A} 39 \mathrm{D} 6465 \mathrm{C} 83 \mathrm{CBB} 2 \mathrm{AF} 79 \mathrm{CCB} 644 \mathrm{Bandrd}=1 \mathrm{andh}=\mathrm{O} 5 \mathrm{fgv} 115 \_\mathrm{m} 5 \mathrm{~Eb}$ MXp32d1hr5w_OWzB6tyMLA3ujD6ZbUandv=1 andr=http\%3a\%2f\%2fwww.bc s.org\%2fcontent\%2fconWebDoc\%2f55892andp=DevEx,5067.1.

Kranz, Johann J., André Hanelt, and Lutz M. Kolbe. "Understanding the influence of absorptive capacity and ambidexterity on the process of business model changethe case of on-premises and cloud-computing software." Information Systems Journal 26.5 (2016): 477-517.

Kupfer, A., Ableitner, L., Schöb, S., and Tiefenbeck, V. (2016). Technology adoption vs. continuous usage intention: do decision criteria change when using a technology?

Kwon, T. H., and Zmud, R. W. (1987, April). Unifying the fragmented models of information systems implementation. In critical issues in information systems research (pp. 227-251). John Wiley and Sons, Inc.

Li, G., Hou, Y., and Wu, A. (2017). Fourth Industrial Revolution: technological drivers, impacts and coping methods. Chinese Geographical Science, 27(4), 626-637. 
Lisitsa, R. S., Sather, D. A., and Hagiu, C. (2008). U.S. Patent No. 7,349,953. Washington, DC: U.S. Patent and Trademark Office.

Luftman, J. N., Lewis, P. R., and Oldach, S. H. (1993). Transforming the enterprise: The alignment of business and information technology strategies. IBM systems journal, 32(1), 198-221.

Luzzini, D., Longoni, A., Moretto, A., Caniato, F., and Brun, A. (2014). Organizing IT purchases: Evidence from a global study. Journal of Purchasing and Supply Management, 20(3), 143-155.

Lyytinen, K., and Rose, G. M. (2003). The disruptive nature of information technology innovations: the case of internet computing in systems development organizations. MIS quarterly, 557-596.

Manuputty, A. D., and Wijaya, A. F. (2013). Information System/Information Technology Strategic Planning in Order Information Technology Development Strategy Using TOGAF (The Open Group Architecture Framework) Methodology in Achieving World Class University in Satya Wacana Christian University. Intelligent Information Management, 5(06), 175.

Majchrzak, A., More, P. H., and Faraj, S. (2012). Transcending knowledge differences in cross-functional teams. Organization Science, 23(4), 951-970.

Matt, C., Hess, T., and Benlian, A. (2015). Digital transformation strategies. Business and Information Systems Engineering, 57(5), 339-343.

Markus, M. L., and Robey, D. (1988). Information technology and organizational change: causal structure in theory and research. Management science, 34(5), 583-598.

Mata, F., Fuerst, W. L., and Barney, J. B. 1995. "Information Technology and Sustained Competitive Advantage: A Resource-Based Analysis," MIS Quarterly (19:4), pp. 487-505

Mauthner, N. S., and Doucet, A. (2003). Reflexive accounts and accounts of reflexivity in qualitative data analysis. Sociology, 37(3), 413-431.

McElheran, K. (2014). Delegation in Multi - Establishment Firms: Evidence from IT Purchasing. Journal of Economics and Management Strategy, 23(2), 225-258.

Merriam, S. B., and Tisdell, E. J. (2015). Qualitative research: A guide to design and implementation. John Wiley and Sons. 
MIS Quarterly (21:2), June 1997, pp. 241-242. MISQ discovery, archival version, June 1997, http://www.misq.org/supplements/. Association for information systems (aisworld) section on qualitative research in information systems, updated version, last modified: june 29, 2020 www.qual.auckland.ac.nz

Monteiro, C., Da Silva, F., and Capretz, L. (2016). The Innovative Behaviour of Software Engineers: Findings from a Pilot Case Study. Proceedings of the 10th ACM/IEEE International Symposium on Empirical Software Engineering and Measurement, $1-10$.

Moore, G. C., and Benbasat, I. (1991). Development of an instrument to measure the perceptions of adopting an information technology innovation. Information systems research, 2(3), 192-222.

Murdoch, D., and Fichter, R. (2017). From doing digital to being digital: Exploring workplace adoption of technology in the age of digital disruption. International Journal of Adult Vocational Education and Technology (IJAVET), 8(4), 13-28.

Myers, M. D., \& Avison, D. (Eds.). (1997). Qualitative research in information systems: a reader. Sage.

Myers, M. D., \& Avison, D. (Eds.). (2002). Qualitative research in information systems: a reader. Sage.

Nicholls, P. T. (1988). Price's square root law: empirical validity and relation to Lotka's Law. Information processing and management, 24(4), 469-477.

Oliveira, T., and Martins, M. F. (2011). Literature review of information technology adoption models at firm level. The Electronic Journal Information Systems Evaluation, 14(1), 110-121.

Orr, B. (2002). The case for web-based procurement. ABA Banking J. 94, 4.

Osmundsen, K., Iden, J., and Bygstad, B. (2018, September). Digital Transformation: Drivers, Success Factors, and Implications. In MCIS (p. 37).

Pervan, G., Bajwa, D., Lewis, L.F. (2005). A study of the adoption and utilization of seven collaboration technologies in large organizations in Australia and New Zealand. Journal of Global Information Technology Management 8 (2), 5-26.

Reich, B. H., and Benbasat, I. (1996). Measuring the linkage between business and information technology objectives. MIS quarterly, 55-81.

Rogers, E. M. (2010). Diffusion of innovations. Simon and Schuster. 
Romanelli, E., and Tushman, M. L. (1994). Organizational transformation as punctuated equilibrium: An empirical test. Academy of Management journal, 37(5), 11411166

Roy, V., Aubert, B.A., 2002.A resource-based analysis of ITsourcing.Database33(2), 29-40.

Sauer, C., Yetton, P. W., and Alexander, L. (1997). Steps to the future: fresh thinking on the management of IT-based organizational transformation. Jossey-Bass Inc., Publishers.

Saldaña, J. (2016). The coding manual for qualitative researchers. Sage

Scholz, R. W., and Tietje, O. (2002). Embedded case study methods: Integrating quantitative and qualitative knowledge. Sage.

Song, Y. (2014). "Bring Your Own Device (BYOD)" for seamless science inquiry in a primary school. Computers and Education, 74, 50-60.

Sriram, V., and Stump, R. (2004). Information technology investments in purchasing: an empirical investigation of communications, relationship, and performance outcomes. Omega, 32(1), 41-55.

Stake, R. E. (1995). The art of case study research. Sage.

Stone, E. F. (1978). Research methods in organizational behavior. Goodyear Publishing Company.

Swanson, E. B. (1994). Information systems innovation among organizations. Management science, 40(9), 1069-1092.

Szajna, B. (1996). Empirical evaluation of the revised technology acceptance model. Management science, 42(1), 85-92.

Tabrizi, B., Lam, E., Girard, K., \& Irvin, V. (2019). Digital transformation is not about technology. Harvard Business Review, 13.

Tallon, P. P. (2007). A process-oriented perspective on the alignment of information technology and business strategy. Journal of Management Information Systems, 24(3), 227-268.

Tarafdar, M., and Qrunfleh, S. (2010). Examining Tactical Information TechnologyBusiness Alignment. Journal of Computer Information Systems, 50(4), 107-116. 
Teece, D. J., Pisano, G., and Shuen, A. 1997. "Dynamic Capabilities and Strategic Management," Strategic Management Journal (18:7), pp. 509-533; Barney, J.B. 1996. "The Resource-Based Theory of the Firm," Organization Science (7:5), pp. 469-469.

Tornatzky, L. G., Fleischer, M., and Chakrabarti, A. K. (1990). Processes of technological innovation. Lexington Books.

Treacy, M., and Wiersema, F. (1993). Customer intimacy and other value disciplines. Harvard business review, 71(1), 84-93.

Tornatzky, L. G., and Klein, K. J. (1982). Innovation Characteristics and Innovation Adoption Implementation: A Meta-Analysis of Findings, IEEE Transactions on Engineering Management (29:1, February), pp. 28-44.

Ullah, A., and Lai, R. (2013). A systematic review of business and information technology alignment. ACM Transactions on Management Information Systems (TMIS), 4(1), 4.

Van Oosterhout, M., Waarts, E., and van Hillegersberg, J. (2006). Change factors requiring agility and implications for IT. European Journal of Information Systems, 15(2), 132-145.

Venkatesh, V., and Davis, F. D. (2000). A theoretical extension of the technology acceptance model: Four longitudinal field studies. Management science, 46(2), 186-204.

Venkatesh, V., Morris, M. G., Davis, G. B., and Davis, F. D. (2003). User acceptance of information technology: Toward a unified view. MIS quarterly, 425-478.

Wagner, I. (2020, January 9). U.S. car sales by year 1951-2018. Retrieved January 10, 2020, from https://www.statista.com/statistics/199974/us-car-sales-since-1951/

Weaver, A. (2018, November 30). What is the optimum size for a tech team? Retrieved January 15, 2020, from https://medium.com/@ andrewweaver/what-is-theoptimum-size-for-a-tech-team-f1181ddfd514

Weill, P., Subramani, M., and Broadbent, M. (2002). Building IT infrastructure for strategic agility. MIT Sloan Management Review, 44(1), 57.

Vial, G. (2019). Understanding digital transformation: A review and a research agenda. The Journal of Strategic Information Systems, 28(2), 118-144. 
Xu, M., David, J. M., and Kim, S. H. (2018). The fourth industrial revolution: opportunities and challenges. International journal of financial research, 9(2), 9095.

Yin, R. K. (2014). Case study research: Design and methods. Sage publications.

Young-Hyman, T. (2017). Cooperating without co-laboring: How formal organizational power moderates cross-functional interaction in project teams. Administrative Science Quarterly, 62(1), 179-214.

Yu, C. S., and Tao, Y. H. (2009). Understanding business-level innovation technology adoption. Technovation, 29(2), 92-109.

Wu, S. P. J., Staub, D., and Liang, T. P. 2015. "How It Governance Mechanisms and Strategic Alignment Influence Organizational Performance: Insights from a Matched Survey of Business and IT Managers," MIS Quarterly (39:2, June)

Zhu, K., Dong, S., Xu, S. X., and Kraemer, K. L. (2006). Innovation diffusion in global contexts: determinants of post-adoption digital transformation of European companies. European journal of information systems, 15(6), 601-616 


\section{APPENDIX A \\ INTERVIEW GUIDE}

The following questions were developed during the Pilot Study and used, unabridged throughout all subsequent case studies.

Q1 What is your job title, and what are your primary responsibilities (describe your day)

Q2 How are decisions made for network equipment purchases?

Q3 How much does your company spend on IT per year?

Q4 What is your network budget?

Q5 What vendors are used as part of your data network?

Q6 What is the typical data network equipment refresh cycle at your company?

Q7 Do you have any vendor certifications (CCNA, MCP, etc.) If so, how long have you had them and why did you get them?

Q8 Do you find vendor certifications useful in your daily job?

Q9 Tell me about your last network refresh. How often do you refresh and why did you refresh last?

Q10. Tell me about some your technology roadmap.

Q11. Tell me about cross functional meetings you have with other business or technology units. 
Q12. What are the short-term strategic initiatives of your company as you understand them?

Q13. What are the long-term strategic initiatives of your company as you understand them?

Q14. What does digital transformation mean to you?

Q15. Are you involved in a digital transformation initiative? If so, please describe the objectives and your role.

Q16 Is the data network ready for digital transformation? Why or why not?

Q17 Tell me about the key challenges in respect to your data network.

Q18 Are many complaints from users about network specific outages? What do users usually complain about in reference to the network?

Q19 What happens when you voice your concerns about the network to your management?

Q20 Please describe current projects or projects you have completed within the last 18 months, which your organization considers to be digital transformation projects. Q21Please describe any other major technology projects on which you are working or have completed within the last 18 months. (If many, take top 5).

Q22. What were the titles of project sponsors for your digitally transformative projects?

Q23. Tell me about your last project that went well. Why did it go well?

Q24. Tell me about your last project that went poorly. Why did it go poorly?

Q25. How did you measure the outcomes from your other major technology projects? 


\section{APPENDIX B}

\section{TABLE OF INTERVIEWEES}

\begin{tabular}{|c|c|c|}
\hline \multicolumn{3}{|c|}{$\begin{array}{l}\text { Table } 17 \\
\text { Table of Interviewees }\end{array}$} \\
\hline & Title & Years with Firm \\
\hline PCS-1 & Chief Information Officer & 25 \\
\hline PCS-1 & Director Project Management & 17 \\
\hline PCS-1 & $\begin{array}{l}\text { Acting Director Networks and } \\
\text { Telecommunications }\end{array}$ & 12 \\
\hline PCS-1 & Network Engineer Level II & 5 \\
\hline PCS-1 & Network Engineer Level II & 8 \\
\hline MCS-1 & Chief Information Officer & 5 \\
\hline MCS-1 & Software Engineer & 2 \\
\hline MCS-1 & Senior Software Engineer & 3 \\
\hline MCS-1 & Senior Network Engineer & 2 \\
\hline MCS-1 & Network and Telecom Architect & 4 \\
\hline MCS-1 & Accounts Payable Specialist & 1 \\
\hline MCS-1 & Logistics Specialist III & 2 \\
\hline MCS-2 & Senior Director of Networking & 2 \\
\hline MCS-2 & Network Manager & 3 \\
\hline MCS-2 & Project Manager & 2 \\
\hline MCS-2 & Project Coordinator & 4 \\
\hline MCS-2 & Senior Network Engineer & 5 \\
\hline MCS-2 & Senior Network Engineer & 4 \\
\hline MCS-2 & Application Developer & 1 \\
\hline MCS-2 & Application Developer & 2 \\
\hline MCS-3 & $\begin{array}{c}\text { Senior Vice President Infrastructure and } \\
\text { Operations }\end{array}$ & 10 \\
\hline MCS-3 & Sr Director Networking & 8 \\
\hline MCS-3 & Senior Network Engineer & 4 \\
\hline MCS-3 & Senior Network Architect & 6 \\
\hline MCS-3 & Senior Network Architect & 1 \\
\hline MCS-4 & Senior Director, Networks & 12 \\
\hline MCS-4 & Principal Network Analyst & 10 \\
\hline MCS-4 & Senior Analyst - Information Technology & 7 \\
\hline MCS-4 & Senior Analyst - Network Technology Systems & 8 \\
\hline MCS-4 & Senior Director - Dispatch Operations Systems & 15 \\
\hline MCS-4 & $\begin{array}{c}\text { Senior Director - Information Technology, } \\
\text { Crew Scheduling }\end{array}$ & 10 \\
\hline
\end{tabular}




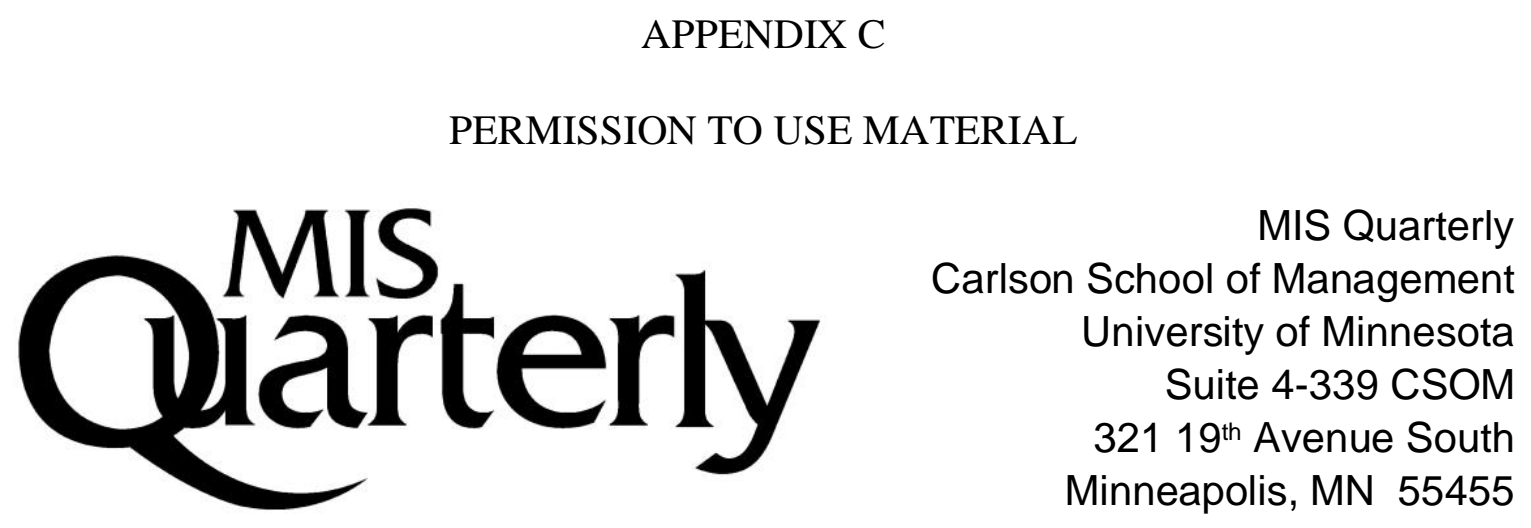

September 15, 2020

Michael Toussaint

Fox School of Business

Temple University

Philadelphia, PA

Permission to use material from

MIS Quarterly in doctoral dissertation

Permission is hereby granted to Michael Toussaint to use an adaptation of Figure 1 (and supporting material as necessary) from "Qualitative Research in Information Systems," M. D. Myers, MIS Quarterly Discovery, June 1997, in his doctoral dissertation, titled "Relational Effects of Data Networks: How Strategically Aligned Data Networks Impact Digital Transformation," for Temple University.

In addition to the citation information for the work, the legend should include

Copyright $\odot$ 1997, Regents of the University of Minnesota. Used with permission.

The permission extends to depositing a copy of the dissertation with Proquest through $\mathrm{UMI} ®$ Dissertation Publishing to sell or provide online the original dissertation, should you choose to list the dissertation with them, but does not extend to future revisions or editions of the dissertation, or publication of the dissertation in any other format.

Janice 1. DeGross

Janice I. DeGross

Manager, MIS Quarterly 